\title{
The Newtonian and Relativistic Theory of Orbits and the Emission of Gravitational Waves
}

\author{
Mariafelicia De Laurentis ${ }^{1,2, *}$ \\ ${ }^{I}$ Dipartimento di Scienze Fisiche, Universitá di Napoli " Federico II",Compl. Univ. di Monte S. Angelo, Edificio G, Via \\ Cinthia, I-80126, Napoli, Italy \\ ${ }^{2}$ INFN Sez. di Napoli, Compl. Univ. di Monte S. Angelo, Edificio G, Via Cinthia, I-80126, Napoli, Italy
}

\begin{abstract}
This review paper is devoted to the theory of orbits. We start with the discussion of the Newtonian problem of motion then we consider the relativistic problem of motion, in particular the post-Newtonian (PN) approximation and the further gravitomagnetic corrections. Finally by a classification of orbits in accordance with the conditions of motion, we calculate the gravitational waves luminosity for different types of stellar encounters and orbits.
\end{abstract}

Keywords: Theroy of orbits, gravitational waves, post-newtonian (PN) approximation, LISA, transverse traceless, Elliptical orbits.

\section{INTRODUCTION}

Dynamics of standard and compact astrophysical bodies could be aimed to unravelling the information contained in the gravitational wave (GW) signals. Several situations are possible such as GWs emitted by coalescing compact binaries-systems of neutron stars (NS), black holes (BH) driven into coalescence by emission of gravitational radiation (considered in the inspiralling, merging and ringdown phases, respectively), hard stellar encounters, and other high-energy phenomena where GW emission is expected. Furthermore the signature of GWs can be always determined by the relative motion of the sources. In this review paper, we want to discuss the problem of how the waveform and the emission of GWs depend on the relative motions in Newtonian, Relativistic and post-Relativistic regimes as for example situations where gravitomagnetic corrections have to be considered in the orbital motion.

As a first consideration, we have to say that the problem of motion, i.e. the problem of describing the dynamics of gravitationally interacting bodies, is the cardinal problem of any theory of gravity. From the publication of Newton's Principia to the beginning of the twentieth century, this problem has been thoroughly investigated within the framework of Newton's dynamics. This approach led to the formulation of many concepts and theoretical tools which have been applied to other fields of physics. As a consequence, the relationship between Einstein's and Newton's theories of gravity has been, and still is, very peculiar. On the one hand, from a theoretical point of view, the existence of Newton's theory facilitated the early development of Einstein's theory by suggesting an approximation method (called post-Newtonian (PN)) which allowed to draw very soon some observational consequences

\footnotetext{
*Address correspondence to this author at the INFN Sez. di Napoli, Compl. Univ. di Monte S. Angelo, Edificio G, Via Cinthia, I-80126, Napoli, Italy; Tel: 0039081676881; E-mail:felicia@na.infn.it
}

of General Relativity (GR). Indeed, the PN approximation method, developed by Einstein himself [1], Droste and de Sitter $[2,3]$ within one year after the publication of GR, led to the predictions of $i$ ) the relativistic advance of perihelion of planets, ii) the gravitational redshift, iii) the deflection of light, iv) the relativistic precession of the Moon orbit, that are the so-called "classical" tests of GR.

On the other hand, as emphasized by Eisenstaedt [4], the use of PN approximation method has had, from a conceptual point of view, the adverse side-effect of introducing implicitly a 'neo-Newtonian' interpretation of GR. Indeed, technically this approximation method follows the Newtonian way of tackling gravitational problems as closely as possible. But this technical reduction of Einstein's theory into the Procrustean bed of Newton's theory surreptitiously entails a corresponding conceptual reduction: the Einstenian problem of motion is conceived within the Newtonian framework of an "absolute" coordinate space and an "absolute" coordinate time. However, some recent developments oblige us to reconsider the problem of motion within Einstein's theory. On the other hand, the discovery of the binary pulsar PSR $1913+16$ by Hulse and Taylor in 1974 [5], and its continuous observation by Taylor and coworkers (see references [6,7]), led to an impressively accurate tracking of the orbital motion of a NS in a binary system. This means that it is worth reconsidering in detail, i.e. at its foundation, the problem of motion also in relation to the problem of generation and detection of GWs. In other words, the motion of sources could give further signatures to GWs and then it has to be carefully reconsidered [8].

The first part of this review paper is devoted to the theory of orbits. The most natural way to undertake this task is starting with the discussion of the Newtonian problem of motion then we consider the relativistic problem of motion, in particular the PN approximation and the further gravitomagnetic corrections. 
The theory of orbits can be connected to GWs since studies of binary systems prove, beyond reasonable doubts, that such a form of radiation has to exist. Detecting the waves directly and exploiting them could result a very impressive way to study astrophysical objects. In other words, the detection of GWs could give rise to the so-called Gravitational Astronomy.

In view of this achievement, it is relevant to stress that GW science has entered a new era. Experimentally ${ }^{1}$, several ground-based laser-interferometer GW detectors (10-1 kHz) have been built in the United States (LIGO) [9], Europe (VIRGO and GEO) [10, 11] and Japan (TAMA) [12], and are now taking data at designed sensitivity.

A laser interferometer space antenna (LISA) $\left(10^{-4}-10^{-2} \mathrm{~Hz}\right)$ might fly within the next decade.

From a theoretical point of view, last years have witnessed numerous major advances. Concerning the most promising GW sources for ground-based and space-based detectors, i.e. binary systems composed of NS, BHs, our understanding of the relativistic two-body problem, and the consequent GW-generation problem, has improved significantly.

Knowledge has also progressed on the problem of motion of a point particle in curved spacetime when the emission of GWs is taken into account (non-geodesic motion) [14, 15]. Solving this problem is of considerable importance for predicting very accurate waveforms emitted by extreme mass-ratio binaries, which are among the most promising sources for LISA [16].

The GW community working at the interface between the theory and the experiment has provided templates [17-19] for binaries and developed robust algorithms [20, 21] for pulsars and other GW-sources observable with ground-based and space-based interferometers. The joined work of data analysts and experimentalists has established astrophysically significant upper limits for several GW sources [22-24] and is now eagerly waiting for the first detection.

In this research framework, searching for criteria to classify how sources collide and interact is of fundamental importance. A first rough criterion can be the classification of stellar encounters in collisional, as in the globular clusters, and in collisionless as in the galaxies [25]. A fundamental parameter is the richness and the density of the stellar system and then, obviously, we expect a large production of GWs in rich and dense systems.

Systems with these features are the globular clusters and the galaxy centers. In particular, one can take into account the stars (early-type and late-type) which are around our Galactic Center, e.g. Sagittarius $A^{*}\left(\operatorname{Sgr} A^{*}\right)$ which could be very interesting targets for the above mentioned groundbased and space-based detectors.

In recent years, detailed information has been achieved for kinematics and dynamics of stars moving in the gravitational field of such a central object. The statistical properties of spatial and kinematical distributions are of particular interest (see e.g. [27-29]). Using them, it is possible to give a quite

${ }^{1} \mathrm{GW}$ experiments started with the pioneering work of Joseph Weber at Maryland in the 60s accurate estimate of the mass and the size of the central object: we have $(2.61 \pm 0.76) \times 10^{6} M_{\odot}$ concentrated within a radius of $0.016 p c$ (about 30 light-days) [30, 31]. More precisely, in [30], it is described a campaign of observations where velocity measurements in the central $\operatorname{arcsec}^{2}$ are extremely accurate. Then from this bulk of data, considering a field of resolved stars whose proper motions are accurately known, one can classify orbital motions and deduce, in principle, the rate of production of GWs according to the different types of orbits.

These issues motivate this review paper in which, by a classification of orbits in accordance with the conditions of motion, we want to calculate the GW luminosity for different types of stellar encounters and orbits (see also [32, 33]).

Following the method outlined in [34, 35], we investigate the GW emission by binary systems in the quadrupole approximation considering bounded (circular or elliptical) and unbounded (parabolic or hyperbolic) orbits. Obviously, the main parameter is the approaching energy of the stars in the system (see also [36] and references therein). We expect that gravitational waves are emitted with a "peculiar" signature related to the encounter-type: such a signature has to be a "burst" wave-form with a maximum in correspondence of the periastron distance. The problem of bremsstrahlung-like gravitational wave emission has been studied in detail by Kovacs and Thorne [37] by considering stars interacting on unbounded and bounded orbits. In this review paper, we face this problem discussing in detail the dynamics of such a phenomenon which could greatly improve the statistics of possible GW sources. For further details see also [26, 38-50].

The review is organized as follows. In Part I, as we said, we discuss the theory of orbits. In Sec.2, we start with the Newtonian theory of orbits and discuss the main features of stellar encounters by classifying trajectories. Sec.3 is devoted to orbits with relativistic corrections. A method for solving the equations of motion of binary systems at the first $\mathrm{PN}$-approximation is reviewed. The aim is to express the solution in a quasi-Newtonian form. In the Sec.4, we study higher order relativistic corrections to the orbital motion considering gravitomagnetic effects. We discuss in details how such corrections come out by taking into account "magnetic" components in the weak field limit of gravitational field. Finally, the orbital structure and the stability conditions are discussed giving numerical examples. Beside the standard periastron corrections of GR, a new nutation effect have to be considered thanks to $c^{-3}$ corrections. The transition to a chaotic behavior strictly depends on the initial conditions. The orbital phase space portrait is discussed.

Part II is devoted to the production and signature of gravitational waves. We start, in Sec. 5 , by deriving the wave equation in linearized gravity and discuss the gauge properties of GWs. Sec.6 is devoted to the problems of generation, emission and interaction of GWs with a detector. In Sect. 7, we discuss the problem of GW luminosity and emission from binary systems moving along Newtonian orbits. The quadrupole approximation is assumed. Sec. 8 is devoted to the same problem taking into account relativistic 
motion. In Sec. 9, also gravitomagnetic effects on the orbits and the emission are considered. In Sec. 10, as an outstanding application of the above considerations, we derive the expected rate of events from the Galactic Center. Due to the peculiar structure of this stellar system, it can be considered a privileged target from where GWs could be detected and classified. Discussion, concluding remarks and perspectives are given in Sec. 11 .

\section{PART I}

\section{THEORY OF ORBITS}

\section{Newtonian Orbits}

We want to describe, as accurately as possible, the dynamics of a system of two bodies, gravitationally interacting, each one having finite dimensions. Each body exerts a conservative, central force on the other and no other external forces are considered assuming the system as isolated from the rest of the universe. Then, we first take into account the non-relativistic theory of orbits since stellar systems, also if at high densities and constituted by compact objects, can be usually assumed in Newtonian regime. In most cases, the real situation is more complicated. Nevertheless, in all cases, it is an excellent starting approximation to treat the two bodies of interest as being isolated from outside interactions. We give here a selfcontained summary of the well-known orbital types [25, 26] which will be extremely useful for the further discussion.

\section{A. Equations of Motion and Conservation Laws}

Newton's equations of motion for two particles of masses $m_{1}$ and $m_{2}$, located at $r_{1}$ and $r_{2}$, respectively, and interacting by gravitational attraction are, in the absence of external forces,

$$
\begin{aligned}
& \frac{d p_{1}}{d t}=-G \frac{m_{1} m_{2}}{\left|r_{1}-r_{2}\right|^{3}}\left(r_{1}-r_{2}\right), \\
& \frac{d p_{2}}{d t}=+G \frac{m_{1} m_{2}}{\left|r_{1}-r_{2}\right|^{3}}\left(r_{1}-r_{2}\right),
\end{aligned}
$$

where $p_{i}=m_{i} \frac{d r_{i}}{d t}$ is the momentum of particle $i,(i=1,2)$, and $G$ is Newtonian gravitational constant.

$$
\frac{d}{d t}\left(p_{1}+p_{2}\right)=0
$$

or, with $P=p_{1}+p_{2}$ denoting the total momentum of the two body system,

$$
P=\text { const. }
$$

Thus we have found a first conservation law, namely the conservation of the total momentum of a two-body system in the absence of external forces. We can make use of this by carrying out a Galilei transformation to another inertial frame in which the total momentum is equal to zero. Indeed, let us apply the transformation

$$
r_{i} \rightarrow r_{i}^{\prime}=r_{i}-v t, \quad i=1,2
$$

hence $p_{i} \rightarrow p_{i}^{\prime}=p_{i}-m_{i} v$ and hence, with $M=m_{1}+m_{2}$,

$$
P \rightarrow P^{\prime}=P-M v
$$

and if we choose $v=\frac{P}{M}$, then the total momentum is equal to zero in the primed frame. We also note that the gravitational force is invariant under the Galilei transformation, since it depends only on the difference $r_{1}-r_{2}$. Thus let us from now on work in the primed frame, but drop the primes for convenience of notation. We can now replace the original equations of motion with the equivalent ones,

$$
P=0, \quad \frac{d p}{d t}=-G \frac{m_{1} m_{2}}{r^{3}} r
$$

where $r=r_{1}-r_{2}, \quad r=|r|$, and $p=p_{1}-p_{2}$. Next we introduce the position vector $R$ of the center of mass of the system:

$$
R=\frac{m_{1} r_{1}+m_{2} r_{2}}{m_{1}+m_{2}}
$$

hence

$$
P=M \frac{d R}{d t}
$$

and hence from $P=0$ we have

$$
R=\text { const } \text {. }
$$

and we can carry out a translation of the origin of our coordinate frame such that $R=0$. The coordinate frame we have arrived at is called center-of-mass frame (CMS). We can also see now that

$$
p=p_{1}=m_{1} \frac{d r_{1}}{d t}=\mu \frac{d r}{d t},
$$

where $\mu=\frac{m_{1} m_{2}}{m_{1}+m_{2}}$ is the reduced mass of the system, and hence the equation of motion can be cast in the form

$$
\mu \frac{d^{2} r}{d t^{2}}=-G \frac{\mu M}{r^{2}} \hat{r}
$$

where we have defined the radial unit vector $\hat{r}=\frac{r}{r}$. We can get two more conservation laws if we take the scalar product of Eq. (2) with $\frac{d r}{d t}$ and its vector product with $r$. The scalar product with $\frac{d r}{d t}$ gives on the left-hand side

$$
\frac{d r}{d t} \cdot \frac{d^{2} r}{d t^{2}}=\frac{1}{2} \frac{d}{d t}\left(\frac{d r}{d t}\right)^{2}
$$

and on the right-hand side we have 


$$
\frac{\hat{r}}{r^{2}} \cdot \frac{d r}{d t}=-\frac{d}{d t}\left(\frac{1}{r}\right)
$$

$$
\frac{p_{r}^{2}}{2 \mu}+\frac{L^{2}}{2 \mu r^{2}}-\frac{\gamma}{r}=E,
$$

hence

$$
\frac{d}{d t}\left(\frac{p^{2}}{2 \mu}-\frac{\gamma}{r}\right)=0
$$

where $\gamma=G \mu M$. This implies that the expression in brackets is conserved, i.e.

$$
\frac{p^{2}}{2 \mu}-\frac{\gamma}{r}=E=\text { const }
$$

Here the first term is the kinetic energy, the second term is the potential energy, and the sum of kinetic energy and potential energy is the total energy $E$, which is a constant of motion. Now take the cross product of Eq. (2) with $r$ : on the right-hand side, we get the cross product of collinear vectors, which is equal to zero, hence

$$
r \times \mu \frac{d}{d t}\left(r \times \frac{d r}{d t}\right)=\frac{d}{d t}(r \times p)=0,
$$

and hence, if we define the angular momentum $L$ by

$$
L=r \times p,
$$

we get the result

$$
\frac{d L}{d t}=0
$$

or

$$
L=\text { const },
$$

i.e. conservation of angular momentum. An immediate consequence of this conservation law is that the radius vector $r$ always stays in one plane, namely the plane perpendicular to $L$. This implies that we can without loss of generality choose this plane as the $(x y)$ coordinate plane. The vector $r$ is then a two-dimensional vector,

$$
r=(x, y)=r \hat{r} . \quad \hat{r}=(\cos \varphi, \sin \varphi),
$$

where we have defined the polar angle $\phi$. With this notation we can express the magnitude of angular momentum as

$$
L=r^{2} \frac{d \phi}{d t},
$$

The conservation of angular momentum can be used to simplify the equation of motion (2.3). To do this we note that

$$
L^{2}=(r \times p)^{2}=r^{2} p^{2}-(r \cdot p)^{2},
$$

hence

$$
p^{2}=\frac{L^{2}}{r^{2}}+p_{r}^{2},
$$

where $p_{r}=\hat{r} \cdot p$ is the radial component of momentum. Substituting into Eq. (2.3) then gives

or, with $p_{r}=\frac{\mu}{d r} d t$,

$$
\frac{1}{2} \mu\left(\frac{d r}{d t}\right)^{2}+\frac{\mathrm{L}^{2}}{2 \mu r^{2}}-\frac{\gamma}{r}=E .
$$

Looking back at our starting point, Eq. (2.1), we reduce the dimensionality of our problem: from the simultaneous differential equations of six functions of time, namely the six components of the position vectors $r_{1}$ and $r_{2}$, we reduce to a pair of simultaneous differential equations for the polar coordinates $r(t)$ and $\phi(t)$ these equations contain two constants of motion, the total energy $E$ and angular momentum $L$. Then a mass $m_{1}$ is moving in the gravitational potential $\Phi$ generated by a second mass $m_{2}$. The vector radius and the polar angle depend on the time as a consequence of the star motion, i.e. $r=r(t)$ and $\phi=\phi(t)$. With this choice, the velocity $v$ of the mass $m_{1}$ can be parameterized as

$$
v=v_{r} \hat{r}+v_{\phi} \hat{\phi},
$$

where the radial and tangent components of the velocity are, respectively,

$$
v_{r}=\frac{d r}{d t}, \quad v_{\varphi}=r \frac{d \varphi}{d t} .
$$

We can split the kinetic energy into two terms where, due to the conservation of angular momentum, the second one is a function of $r$ only. An effective potential energy $V_{\text {eff }}$,

$$
V_{e f f}=\frac{\mathrm{L}^{2}}{2 \mu r^{2}}-\frac{\gamma}{r}
$$

is immediately defined. The first term corresponds to a repulsive force, called the angular momentum barrier. The second term is the gravitational attraction. The interplay between attraction and repulsion is such that the effective potential energy has a minimum. Indeed, differentiating with respect to $r$ one finds that the minimum lies at $r_{0}=\frac{L^{2}}{\gamma \mu}$ and that

$$
V_{e f f}^{\min }=-\frac{\mu \gamma^{2}}{2 L^{2}} .
$$

Therefore, since the radial part of kinetic energy,

$$
K_{r}=\frac{1}{2} \mu\left(\frac{d r}{d t}\right)^{2},
$$

is non-negative, the total energy must be not less than $V_{\text {eff }}^{\min }$, i.e. 
Table1. Orbits in Newtonian Regime Classified by the Approaching Energy

\begin{tabular}{|c|c|c|}
\hline$C=0$ & $E=E_{\min }$ & Circular Orbits \\
\hline \hline $0<|C|<\frac{\gamma \mu}{L^{2}}$ & $E_{\min }<E<0$ & elliptic orbits \\
\hline$|C|=\frac{\gamma \mu}{L^{2}}$ & $E=0$ & parabolic orbits \\
\hline$|C|>\frac{\gamma \mu}{L^{2}}$ & $E>0$, & hyperbolic orbits \\
\hline
\end{tabular}

$$
E \geq E_{\min }=-\frac{\mu \gamma^{2}}{2 L^{2}}
$$

The equal sign corresponds to the radial motion. For $E_{\min }<E<0$, the trajectory lies between a smallest value $r_{\min }$ and greatest value $r_{\max }$ which can be found from the condition $E=V_{e f f}$, i.e.

$$
r_{\{\text {min, } \max \}}=-\frac{\gamma}{2 E} \pm \sqrt{\left(\frac{\gamma}{2 E}\right)^{2}+\frac{L^{2}}{2 \mu E}},
$$

where the upper (lower) sign corresponds to $r_{\max }\left(r_{\min }\right)$. Only for $E>0$, the upper sign gives an acceptable value; the second root is negative and must be rejected. Let us now proceed in solving the differential equations (2.4) and (2.5). We have

$$
\frac{d r}{d t}=\frac{d r}{d \phi} \frac{d \phi}{d t}=\frac{L}{\mu r^{2}} \frac{d r}{d \phi}=-\frac{L}{\mu} \frac{d}{d \phi}\left(\frac{1}{r}\right),
$$

and defining, as standard, the auxiliary variable $u=1 / r$, Eq. (2.5) takes the form

$$
u^{\prime 2}+u^{2}-\frac{2 \gamma \mu}{L^{2}} u=\frac{2 \mu E}{L^{2}},
$$

where $u^{\prime}=d u / d \phi$ and we have divided by $L^{2} / 2 \mu$. Differentiating with respect to $\phi$, we get

$$
u^{\prime}\left(u^{\prime \prime}+u-\frac{\gamma \mu}{L^{2}}\right)=0
$$

hence either $u^{\prime}=0$, corresponding to the circular motion, or

$$
u^{\prime \prime}+u=\frac{\gamma \mu}{L^{2}}
$$

which has the solution

$$
u=\frac{\gamma \mu}{L^{2}}+C \cos (\varphi+\alpha)
$$

or, reverting the variable,

$$
r=\left[\frac{\gamma \mu}{L^{2}}+C \cos (\varphi+\alpha)\right]^{-1}
$$

which is the canonical form of conic sections in polar coordinates [51]. The constant $C$ and $\alpha$ are two integration constants of the second order differential equation (2.8). The solution (2.9) must satisfy the first order differential equation (2.7). Substituting (2.9) into (2.7) we find, after a few algebra,

$$
C^{2}=\frac{2 \mu E}{L^{2}}+\left(\frac{\gamma \mu}{L^{2}}\right)^{2},
$$

and therefore, taking account of Eq. (IIA), we get $C^{2} \geq 0$. This implies the four kinds of orbits given in Table IIA and in Fig. (1).

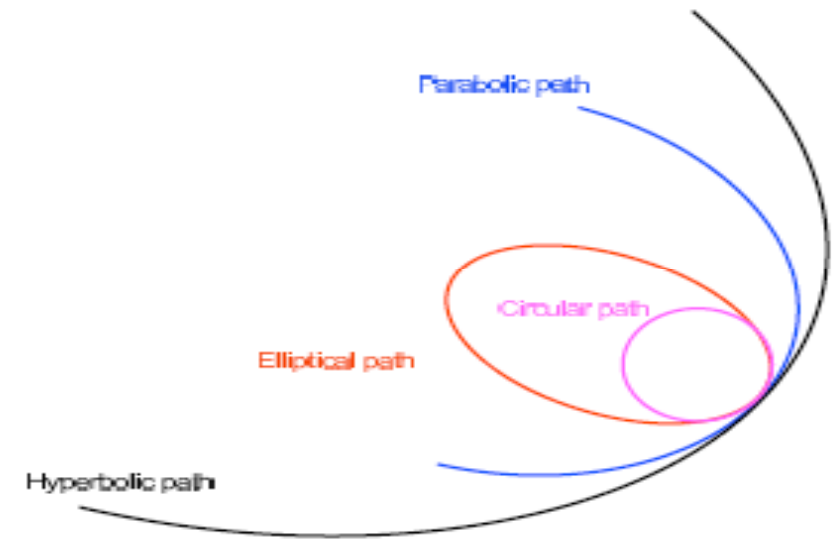

Fig. (1). Newtonian paths: in black line we have hyperbolic path, in blue line we have parabolic path, in red line the elliptical path and in ciano the circular path.

\section{B. Circular Orbits}

Circular motion corresponds to the condition $u^{\prime}=0$ from which one find $r_{0}=L^{2} / \mu \gamma$ where $V_{\text {eff }}$ has its minimum. We also note that the expression for $r_{0}$ together with Eq. (II A) gives

$$
r_{0}=-\frac{\gamma}{2 E_{\min }}
$$

Thus the two bodies move in concentric circles with radii, inversely proportional to their masses and are always in opposition.

\section{Elliptical Orbits}

For $0<|C|<\mu \gamma / L^{2}, r$ remains finite for all values of $\phi$. Since $r(\phi+2 \pi)=r(\phi)$, the trajectory is closed and it is an ellipse. If one chooses $\alpha=0$, the major axis of the ellipse corresponds to $\phi=0$. We get

$$
r_{\mid \varphi=0}=r_{\text {min }}=\left[\frac{\gamma \mu}{L^{2}}+C\right]^{-1},
$$

and

$$
r_{\mid \varphi=\pi}=r_{\max }=\left[\frac{\gamma \mu}{L^{2}}-C\right]^{-1},
$$


and since $r_{\max }+r_{\text {min }}=2 a$, where $a$ is the semi-major axis of the ellipse, one obtains

$$
a=r_{\mid \varphi=0}=r_{\min }=\frac{\gamma \mu}{L^{2}}\left[\left(\frac{\gamma \mu}{L^{2}}\right)^{2}+C^{2}\right]^{-1}
$$

$C$ can be eliminated from the latter equation and Eq. (2.10) and then

$$
a=-\frac{\gamma}{2 E}
$$

Furthermore, if we denote the distance $r_{\mid \varphi=\pi / 2}$ by $l$, the so-called semi-latus rectum or the parameter of the ellipse, we get

$$
l=\frac{L^{2}}{\gamma \mu}
$$

and hence the equation of the trajectory

$$
r=\frac{l}{1+\varepsilon \cos \varphi}
$$

where $\varepsilon=\sqrt{\frac{1-l}{a}}$ is the eccentricity of the ellipse. If we consider the major semiaxis of orbit $a$ Eq. (2.12) and the eccentric anomaly $\varepsilon$, the orbit can be written also as (see [52])

$$
r=a(1-\varepsilon \cos E)
$$

this equation, known as Kepler's equation, is transcendental in $\varepsilon$, and the solution for this quantity cannot expressed in a finite numbers of terms. Hence, there is the following relation between the eccentric anomaly and the angle $\phi$ :

$$
\cos \phi=\frac{\cos \varepsilon-\varepsilon}{1-\varepsilon \cos \varepsilon}
$$

\section{Parabolic and Hyperbolic Orbits}

These solutions can be dealt together. They correspond to $E \geq 0$ which is the condition to obtain unbounded orbits. Equivalently, one has $|C| \geq \gamma \mu / L^{2}$.

The trajectory is

$$
r=l(1+\varepsilon \cos \phi)^{-1}
$$

where $\varepsilon \geq 1$. The equal sign corresponds to $E=0$. Therefore, in order to ensure positivity of $r$, the polar angle $\phi$ has to be restricted to the range given by

$$
1+\varepsilon \cos \phi>0
$$

This means $\cos \varphi>-1$, i.e. $\phi \in(-\pi, \pi)$ and the trajectory is not closed any more. For $\varphi \rightarrow \pm \pi$, we have $r \rightarrow \infty$. The curve (2.15), with $\varepsilon=1$, is aparabola. For $\varepsilon>1$, the allowed interval of polar angles is smaller than $\phi \in(-\pi, \pi)$, and the trajectory is a hyperbola. Such trajectories correspond to non-returning objects. Let us consider a semiaxis $a$ and $F$ as variable, analogous to the elliptic eccentric anomaly $E$. The hyperbolic orbit is defined also by

$$
r=a(\varepsilon \cosh F-1),
$$

hence, there is the following relation between $F$ and the angle $\phi$ :

$$
\cos \phi=\frac{l-a(\varepsilon \cosh F-1)}{\varepsilon a(\varepsilon \cosh F-1)} .
$$

Finally the parabolic orbit can be defined by the another relation (see [52])

$$
r=\frac{P^{2}}{2}
$$

where $\mathrm{P}$ is a parameter. In this case

$$
\cos \phi=\frac{2 l-P^{2}}{P^{2}}
$$

As we will discuss below, this classification of orbital motions can reveal extremely useful for the waveform signature of gravitational radiation. Let us now take into account the relativistic theory of orbits.

\section{RELATIVISTIC ORBITS}

As we have seen in the above Section, the non-relativistic two bodies problem consists in two sub-problems:

1. Deriving the equations of orbital motion for two gravitationally interacting extended bodies,

\section{Solving these equations of motion.}

In the case of widely separated objects, one can simplify the sub-problem by neglecting the contribution of the quadrupole and higher multipole momenta of the bodies to their external gravitational field, thereby approximating the equations of orbital motion of two extended bodies by the equations of motion of two point masses located at the Newtonian center of mass of the extended objects. Then the sub-problem can be exactly solved as shown in the above Section. The two body problem in GR is more complicated: because of the non-linear hyperbolic structure of Einstein's field equations, one is not sure of the good formulation of boundary conditions at infinity, so that the problem is not even well posed [53]. Moreover, since in Einstein's theory the local equations of motion are contained in the gravitational field equations, it is a priori difficult to separate the problem in two sub-problems, as in the non-relativistic case, where one can compute the gravitational field as a linear functional of the matter distribution independently of its motion. Furthermore, even when one can achieve such separation and derive some equations of orbital motion for the two bodies, these equations will a priori not be ordinary differential equations but, because of the finite velocity of propagation of gravity, will consist in some kind of retarded integro-differential system [54]. However, all these difficulties can be somehow dealt with if one resorts to approximation procedures and breaks the general covariance by selecting special classes of coordinates systems [55]. 
Two physically different situations, amenable to perturbation treatments, have been considered in the literature:

1. The problem of two weakly self-gravitating, slowly moving, widely separated fluid bodies which has been treated by the so-called PN approximation schemes (for references see [38, 43, 56, 57]),

2. The problem of two strongly self-gravitating, widely separated bodies which has been treated by matching a strong field "internal" approximation scheme in and near the objects to a weak field "external" approximations scheme outside the objects.

The approach has been pursued both for slowly moving objects, either BHs [58] or in general strongly selfgravitating objects [59], and for strongly self-gravitating objects moving with arbitrary velocities $[60,61]$. In the latter case, equations of orbital motion were considered in the form of a retarded-integro-differential system which however could be transformed into ordinary differential equations and which, when attention was restricted to slowly moving bodies, were expanded in power series of $\frac{v}{c}[61,62]$. When keeping only the first relativistic corrections to Newton's law (first post-Newtonian approximation), it turns out that the equations of orbital motion of widely separated, slowly moving, strongly self-gravitating objects depend only on two parameters (the Schwarzschild masses) and are identical to the equations of motion of weakly self-gravitating objects (when using, in both cases, a coordinate system which is harmonic at lowest order). This is, in fact, a non-trivial consequence of the structure of Einstein's theory [54]. Then, in the next subsections, we consider the PN motion including secular and periodic effects at first order approximation and we shall show that the equations of motion can be written in a quasi-Newtonian form.

\section{A. Relativistic Motion and Conservation Laws}

The relativistic case can be seen as a correction to the Newtonian theory of orbits $[54,62]$. In GR, the time is incorporate as a mathematical dimension, so that the fourdimensional rectangular perifocal coordinates are $(x, y, z, t)$ and the four dimensional polar coordinates are $(r, \theta, \varphi, t)$. The first post Newtonian equations of orbital motion of a binary system constrain the evolution in coordinate time $t$ of the positions $r_{1}$ and $r_{2}$ of the two objects. These positions represent the center of mass in the case of weakly selfgravitating objects (see e.g. $[54,57]$ ) and the center of field in the case of strongly self gravitating objects (see [61]). They can be derived from Lagrangian which is function of the simultaneous position $r_{1}(t), r_{2}(t)$, and velocities $v_{1}(t)=\frac{d r}{d t}$ and $v_{2}(t)=\frac{d r_{2}}{d t}$ in a given harmonic coordinate system, and of two constant parameters, the Schwarzschild masses of the objects $m_{1}$ and $m_{2}$ :

$$
L_{P N}\left(r_{1}(t), r_{2}(t), v_{1}(t), v_{2}(t)\right)=L_{N}+\frac{1}{c^{2}} L_{2},
$$

with

$$
L_{N}=\frac{1}{2} m_{1} v_{1}^{2}+\frac{1}{2} m_{2} v_{2}^{2}+\frac{G m_{1} m_{2}}{R},
$$

and

$$
\begin{aligned}
& L_{2}=\frac{1}{8} m_{1} v_{1}^{4}+\frac{1}{8} m_{2} v_{2}^{4}+ \\
& +\frac{G m_{1} m_{2}}{2 R}\left[3\left(v_{1}^{2}+v_{2}^{2}\right)-7\left(v_{1} v_{2}\right)-N^{2} v_{1} v_{2}-G \frac{M}{R}\right]
\end{aligned}
$$

where we have introduced the instantaneous relative position vector $R=r_{1}-r_{2}$ and $R=|R|$ while $N=\frac{R}{R}$. In (3.1) and (3.2) we used the short notations: $v_{1} \cdot v_{1}=\left|v_{1}\right|=v_{1}^{2}$, $v_{1} \cdot v_{2}=v_{1} v_{2}$ for the ordinary Euclidean scalar products, and $c$ is the velocity of light. The invariance, at the PN approximation, and modulo an exact time derivative, of $L_{P N}$ under spatial translations and Lorentz boosts implies, via Noether's theorem, the conservation of the total linear momentum of the system:

$$
P_{P N}=\frac{\partial L_{P N}}{\partial v_{1}}+\frac{\partial L_{P N}}{\partial v_{2}}
$$

and of the relativistic center of mass integral

$$
\begin{aligned}
K_{P N} & =G_{P N}-t P_{P N}, \\
G_{P N} & =\sum\left(m_{1}+\frac{1}{2} \frac{m_{1} v^{2}}{c^{2}}-\frac{1}{2} \frac{G m_{1} m_{2}}{R c^{2}}\right) r,
\end{aligned}
$$

the sum is over the two objects [57, 62]. By a Poincaré transformation it is possible to get a PN center of mass frame where $P_{P N}=K_{P N}=0$. In this frame one has:

$$
\begin{aligned}
& r_{1}=\frac{\mu}{m_{1}} R+\frac{\mu\left(m_{1}-m_{2}\right)}{2 M^{2} c^{2}}\left(V^{2}-\frac{G M}{R}\right) R, \\
& r_{2}=-\frac{\mu}{m_{2}} R+\frac{\mu\left(m_{1}-m_{2}\right)}{2 M^{2} c^{2}}\left(V^{2}-\frac{G M}{R}\right) R,
\end{aligned}
$$

where $V=\frac{d R}{d t}=v_{1}-v_{2}$ is the istantaneous relative velocity.

The problem of solving the motion of the binary system is then reduced to the simpler problem of solving the relative motion in the PN center of mass frame. For the sake of completeness, let us write down these equations of motion derived from (3.1)-(3.2), and where, after variation, the positions and velocities are replaced by their center of mass expressions (3.4): 


$$
\begin{aligned}
& \frac{d V}{d t}=\frac{G M}{2 c^{2} R^{3}}\left[4 G M N(v+2)-R\left(2 N c^{2}+\right.\right. \\
& \left.\left.+4(N V) V(v-2)-3(N V)^{2} N v+2 N V^{2}(3 v+1)\right)\right],
\end{aligned}
$$

where we have introduced a mass parameter $v=\frac{\mu}{M}=\frac{m_{1} m_{2}}{\left(m_{1}+m_{2}\right)^{2}}$ with $\left(0 \leq v \leq \frac{1}{4}\right)$. At this point it is worth to notice that in spite of the fact that it is in general incorrect to use, before variation, in a Lagrangian a consequence, like Eq. (3.4), of the equations of motions, which are obtained only after variation, it turns out that the relative motion in PN center of mass frame, Eq. (3.5), can be correctly derived from a Lagrangian obtained by replacing in the total Lagrangian (divided by $\mu$ ) $\frac{1}{\mu} L_{P N}\left(r_{1}, r_{2}, v_{1}, v_{2}\right)$ the positions and velocities by their PN center of mass expressions obtained from (3.4) and that moreover it is even sufficient to use the non-relativistic center of mass expressions:

$$
\begin{aligned}
& r_{1 N}=\frac{\mu}{m_{1}} R, \\
& r_{2 N}=\frac{\mu}{m_{2}} R, \\
& v_{1 N}=\frac{\mu}{m_{1}} V, \\
& v_{2 N}=\frac{\mu}{m_{2}} V .
\end{aligned}
$$

The proof goes as follows [54]. Let us introduce the following linear change spatial variables in the PN Lagrangian $L_{P N}\left(r_{1}-r_{2}, \frac{r_{1}}{d t}, \frac{r_{2}}{d t}\right):\left(r_{1}, r_{2}\right) \rightarrow(R, X)$ with $R=r_{1}-r_{2}$ and $X=\frac{\left(m_{1} r_{1}+m_{2} r_{2}\right)}{M}$, that is:

$$
\begin{aligned}
& r_{1}=r_{1 N}+X, \\
& r_{2}=r_{2 N}+X,
\end{aligned}
$$

which implies (denoting $\frac{d X}{d t}=W$ ):

$$
\begin{aligned}
& v_{1}=v_{1 N}+W, \\
& v_{2}=v_{2 N}+W .
\end{aligned}
$$

Expressing

$$
L_{P N}=L_{N}\left(r_{1}-r_{2}, v_{1}, v_{2}\right)+\left(\frac{1}{c^{2}}\right) L_{2}\left(r_{1}-r_{2}, v_{1}, v_{2}\right),
$$

given by Eq. (3.1)-(3.2) in terms of the new variables one finds:

$$
\begin{aligned}
\mathrm{L}_{P N} & =\frac{1}{2} M W^{2}+\frac{1}{2} \mu V^{2}+\frac{G \mu M}{R}+ \\
& +\frac{1}{c^{2}} L_{2}\left(R \frac{\mu V}{m_{1}}+W,-\frac{V}{m_{2}}+W\right) .
\end{aligned}
$$

Hence one obtains as a consequence of the equations of the PN motion:

$$
\begin{aligned}
& \mathrm{O}=\frac{1}{\mu} \frac{\delta \mathrm{L}_{P N}}{\delta R}=\left(\frac{\partial}{\partial R}-\frac{d}{d t} \frac{\partial}{\partial R}\right)\left[\frac{1}{2} V^{2}+\frac{G M}{R}+\right. \\
& \left.+\frac{1}{\mu c^{2}} \mathrm{~L}_{2}\left(R, \frac{\mu}{m_{1}} V+W,-\frac{\mu}{m_{2}} V+W\right)\right],
\end{aligned}
$$

where in the last bracket we have discarded $\frac{1}{2} M W^{2}$ which gives no contribution. The first two terms in the ( $r h s)$ of Eq. (3.8) yield the Newtonian relative motion. We wish to evaluate the relativistic corrections to the relative motion: $\left(\frac{\delta}{\delta R}\right)\left(\frac{\mathrm{L}}{\mu c^{2}}\right)$ in the PN center of mass frame. Now $L_{2}$ is a polynomial in the velocities and therefore a polynomial in $W$, and from Eq. (3.4) one sees that in the PN center of mass frame $W=\mathrm{O}\left(\frac{1}{c^{2}}\right)$. Therefore as $\frac{\delta}{\delta R}$ does not act on $W$, we see that the contributions coming from $W$ to the $r h s$ of Eq. (3.8) are of the second PN order $\mathrm{O}\left(\frac{1}{c^{4}}\right)$ that we shall consistently neglect throughout this work. In other words one obtains as a consequence of the equations of the PN motion in the PN center of mass frame:

$$
\begin{aligned}
& \frac{\delta}{\delta R}\left[\frac{1}{2} V^{2}+\frac{G M}{R}+\frac{1}{\mu c^{2}} \mathrm{~L}_{2}\left(R, \frac{\mu}{m_{1}} V,-\frac{\mu}{m_{2}} V\right)\right] \\
& =O\left(\frac{1}{c^{4}}\right) .
\end{aligned}
$$

This shows that the equations of the relative motion in the PN center of mass frame derive from the following Lagrangian:

$$
\mathrm{L}_{P N}^{R}(R, V)=\frac{1}{2} V^{2}+\frac{G M}{R}+\frac{1}{\mu c^{2}} \mathrm{~L}_{2}\left(R, \frac{\mu}{m_{1}} V,-\frac{\mu}{m_{2}} V\right),
$$

which happens to be obtained by replacing in the full PN Lagrangian, see Eq. (3.7) above, $X$ and $W$ by zero, i. e. the original variables by Eq. (3.6) and by dividing by $\mu$ [54]. The explicit expression of $L_{P N}^{R}$ reads:

$$
\begin{aligned}
& L_{P N}^{R}(R, V)=\frac{1}{2} V^{2}+\frac{G M}{R}+\frac{1}{8}(1-3 v) \frac{V^{4}}{c^{2}}+ \\
& \frac{G M}{2 R c^{2}}\left[(3+v) V^{2}+v(N V)^{2}-\frac{G M}{R}\right] .
\end{aligned}
$$


The Lagrangian (3.9) was obtained in [63]. The integration of the equations (3.5) can be done in several different ways.

- A standard approach: Lagrange's method of variation the osculating elements.

- The Hamilton-Jacobi equation approach which, takes advantage of the existence of the PN Lagrangian is the route which has been taken by Landau and Lifshits [26], who worked out only the secular precession of the periastron.

- Another approach, based on the Maupertuis principle ${ }^{2}$, which reduces the $\mathrm{PN}$ problem to a simple auxiliary Newtonian problem.

To describe the motion, it is convenient to use the standard method to solve the non-relativistic two-bodies problem and which consists in exploiting the symmetries of the relative Lagrangian $L_{P N}^{R}$. The invariance $L_{P N}^{R}$ under time translations and space rotations implies the existence of four first integrals:

$$
\begin{aligned}
& E=V \cdot \frac{\partial L_{P N}^{R}}{\partial V}-L_{P N}^{R} \text { and } J=R \times \frac{\partial L_{P N}^{R}}{\partial V}: \\
& E=\frac{1}{2} V^{2}-\frac{G M}{R}+\frac{3}{8}(1-3 v) \frac{V^{4}}{c^{2}}+ \\
& \frac{G M}{2 R c^{2}}\left[(3+v) V^{2}+v(N V)^{2}-\frac{G M}{R}\right] \\
& J=R \times V\left[1+\frac{1}{2}(1-3 v) \frac{V^{2}}{c^{2}}+(3+v) \frac{G M}{2 R c^{2}}\right]
\end{aligned}
$$

It is checked that these quantities coincide respectively with $\mu^{-1}$ times the total Noether energy and the total Noether angular momentum of the binary system when computed in the PN center of mass frame [64]. Eq. (3.11) implies that the motion takes place in a coordinate plane, therefore one can introduce polar coordinates $R=r$ and $\phi$ in the plane (i.e. $\left.r_{x}=r \cos \phi, r_{y}=r \sin \phi, r_{z}=0\right)$ ). Then starting from the first integrals (3.10)-(3.11) and using the identities: $\quad V^{2}=\left(\frac{d r}{d t}\right)^{2}+r^{2}\left(\frac{d \phi}{d t}\right)^{2}, \quad R \times V \mid=r^{2} \frac{d \phi}{d t}$, $N V=\frac{d r}{d t}$, we obtain by iteration ${ }^{3}$

$$
\left(\frac{d r}{d t}\right)^{2}=A+\frac{2 B}{r}+\frac{C}{r^{2}}+\frac{D}{r^{3}}
$$

\footnotetext{
${ }^{2}$ In classical mechanics, Maupertuis' principle is an integral equation that determines the path followed by a physical system without specifying the time parameterization of that path. It is a special case of the more generally stated principle of least action. More precisely, it is a formulation of the equations of motion for a physical system not as differential equations, but as an integral equation, using the calculus of variations.

${ }^{3}$ In these and the following equations we neglect terms of the second PN order $\mathrm{O}\left(\frac{1}{c^{4}}\right)$.
}

$$
\frac{d \varphi}{d t}=\frac{H}{r^{2}}+\frac{I}{r^{3}},
$$

where the coefficients $A, B, C, D, H, I$ are the following polynomials in $E$ and $J=|J|$ :

$$
\begin{aligned}
& A=2 E\left(1+\frac{3}{2}(3 v-1) \frac{E}{c^{2}}\right), \\
& B=G M\left(1+(7 v-6) \frac{E}{c^{2}}\right), \\
& C=-J^{2}\left(1+2(3 v-1) \frac{E}{c^{2}}\right)+(5 v-10) \frac{G^{2} M^{2}}{c^{2}}, \\
& D=(-3 v+8) \frac{G M J^{2}}{c^{2}}, \\
& H=J\left(1+(3 v-1) \frac{E}{c^{2}}\right), \\
& I=(2 v-4) \frac{G M J}{c^{2}} .
\end{aligned}
$$

The relativistic "relative motion", i.e. the solution of Eq. (3.12) can be simply reduced to the integration of auxiliary non-relativistic radial motion. Indeed let us consider the following change of the radial variable:

$$
r=\bar{r}+\frac{D}{2 C_{0}}
$$

where $C_{0}$ is the limit of $C$ when $c^{-1} \rightarrow 0$ with $\left(C_{0}=-J^{2}\right)$. Geometrically, the transformation which is expressed in polar coordinates by the equation: $r^{\prime}=r+$ const,$\phi^{\prime}=\phi$, is called aconchoidal transformation [54]. Taking into account the fact that $D$ is $\mathrm{O}\left(\frac{1}{c^{2}}\right)$ and that we can neglect all terms of order $\mathrm{O}\left(\frac{1}{c^{4}}\right)$, we find that replacing Eq. (3.15) in Eqs. (3.1)-(3.3), leads to:

$$
\left(\frac{d \bar{r}}{d t}\right)^{2}=A+\frac{2 B}{\bar{r}}+\frac{\bar{C}}{\bar{r}^{2}}
$$

with

$$
\bar{C}=C-\frac{B D}{C_{0}} .
$$

Then, in the case of quasi-elliptical motion $(E<0 ; A<0)$, $\bar{r}$ is a linear function of $\cos E, E$ being an eccentric anomaly and the same is true of $r=\bar{r}+\frac{D}{2 C_{0}}$. We then obtain the PN radial motion in quasi-Newtonian parametric form ( $t_{0}$ being a constant of integration): 


$$
\begin{array}{r}
n\left(t-t_{0}\right)=E-\varepsilon_{t} \sin E, \\
r=a_{r}\left(1-\varepsilon_{r} \cos E\right),
\end{array}
$$

with

$$
\begin{aligned}
& n=\frac{(-A)^{3 / 2}}{B}, \\
& \varepsilon_{t}=\left[1 \frac{A}{B^{2}}\left(C-\frac{B D}{C_{0}}\right)\right]^{1 / 2}, \\
& a_{r}=-\frac{A}{B}+\frac{D}{2 C_{0}}, \\
& \varepsilon_{r}=\left(1+\frac{A D}{2 B C_{0}}\right) \varepsilon_{t} .
\end{aligned}
$$

The main difference between the relativistic radial motion and the non-relativistic one is the appearence of two eccentricities: the time eccentricity $\varepsilon_{t}$ appearing in the Kepler equation (3.17) and the relative radial eccentricity $\varepsilon_{r}$. Using (3.14) we can express $a_{r}, \varepsilon_{r}, \varepsilon_{t}$ and $n$ in terms of $E$ and $J$ :

$$
\begin{aligned}
& a_{r}=\frac{G M}{E}\left[1-\frac{1}{2}(v-7) \frac{E}{c^{2}}\right], \\
& \varepsilon_{r}=\left\{1+\frac{2 E}{G^{2} M^{2}}\left[1+\left(\frac{5}{2} v-\frac{15}{2}\right) \frac{E}{c^{2}}\right]\right. \\
& \left.\left[J^{2}+(v-6) \frac{G^{2} M^{2}}{c^{2}}\right]\right\}^{1 / 2}, \\
& \varepsilon_{t}=\left\{1+\frac{2 E}{G^{2} M^{2}}\left[1+\left(-\frac{7}{2} v-\frac{17}{2}\right)\right]\right. \\
& \left.\left[J^{2}+(-2 v+2) \frac{G^{2} M^{2}}{c^{2}}\right]\right\}, \\
& n=\frac{(-2 E)^{3 / 2}}{G M}\left[1+\frac{1}{4}(v-15) \frac{E}{c^{2}}\right] .
\end{aligned}
$$

It is remarkable that a well known result of the Newtonian motion is still valid at PN level: both the relative semi-major axis $a_{r}$ the mean motion $n$ depend only on the center of mass energy $E$. The same is true for the time of return to periastron period $P=\frac{2 \pi}{n}$. As a consequence we can also express $n$ in term of $a_{r}$ :

$$
n=\left(\frac{G M}{a_{r}^{3}}\right)^{1 / 2}\left[1+\frac{G M}{2 a_{r} c^{2}}(-9+v)\right]
$$

Let us note also that the relationships between $e_{r}$ and $e_{t}$ are:

$$
\begin{aligned}
& \frac{\varepsilon_{r}}{\varepsilon_{t}}=1+(3 v-8) \frac{E}{c^{2}}, \\
& \frac{\varepsilon_{r}}{\varepsilon_{t}}=1+\frac{G M}{2} a_{r} c^{2}\left(4-\frac{3}{2} v\right) .
\end{aligned}
$$

The relativistic angular motion, i.e. the solution of Eq. (3.13) can also be simply reduced to the integration of an auxiliary non relativistic angular motion. Let us first make, at $O\left(\frac{1}{c^{2}}\right)$ order, the following conchoidal transformation:

$$
r=\tilde{r}+\frac{I}{2 H},
$$

which transforms Eq. (3.13) into

$$
\frac{d \phi}{d t}=\frac{H}{\tilde{r}^{2}},
$$

where $\tilde{r}$ can be expressed as

$$
\tilde{r}=\tilde{a}(1-\tilde{\varepsilon} \cos E) .
$$

Let us note also the relationship between $e_{r}$ and $e_{t}$ :

$$
\begin{aligned}
& \tilde{a}=a_{r}-\frac{I}{2 H}, \\
& \tilde{\varepsilon}=\varepsilon_{r}\left(1-\frac{A I}{2 B H}\right) .
\end{aligned}
$$

The differential time is given, from Eq. (3.17) by:

$$
d t=n^{-1}\left(1-\varepsilon_{t} \cos E\right) d E .
$$

Hence we get

$$
d \phi=\frac{H}{n \tilde{a}^{2}} \frac{\left(1-\varepsilon_{t} \cos E\right)}{(1-\tilde{\varepsilon} \cos E)^{2}} d E .
$$

As can be seen from Eq. (3.17) and Eq. (3.22) $\varepsilon_{t}$ and $\tilde{\varepsilon}$ differ by only small terms of order $\frac{1}{c^{2}}$. Now if we introduce any new eccentricity say $\varepsilon_{\varphi}$ also very near $\varepsilon_{t}$ so that we can write: $\varepsilon_{t}=\frac{1}{\left(\varepsilon_{t}+\varepsilon_{\varphi}\right)} 2+\varepsilon, \quad \varepsilon_{\varphi}=\frac{\left(\varepsilon_{t}+\varepsilon_{\varphi}\right)}{2}-\varepsilon$, with $\varepsilon=0\left(\frac{1}{c^{2}}\right)$ then

$$
\left(1-\varepsilon_{t} \cos E\right)\left(1-\varepsilon_{\varphi} \cos E\right)=\left(1-\frac{\left(\varepsilon_{t}+\varepsilon_{\varphi}\right)}{2} \cos E\right)^{2}-\varepsilon^{2} \cos ^{2} E .
$$

Therefore if we choose $\varepsilon_{\varphi}$ such that the average of $\varepsilon_{t}$ and $\varepsilon_{\varphi}$ is equal to $\tilde{\varepsilon}$ i.e. $\varepsilon_{\varphi}=2 \tilde{\varepsilon}-\varepsilon_{t}$ we have

$$
\frac{\left(1-\varepsilon_{t} \cos E\right)^{2}}{(1-\tilde{\varepsilon} \cos E)}=\frac{1}{1-\varepsilon_{\varphi} \cos E}+O\left(\frac{1}{c^{4}}\right)
$$


which transforms Eq. (III A) into a Newtonian like angular motion equation

$$
d \phi=\frac{H}{n \tilde{a}^{2}} \frac{d E}{1-\varepsilon_{\phi} \cos E}
$$

which is easily integrated to give

$$
\phi-\phi_{0}=K A_{\varepsilon_{\phi}}(E)
$$

$\phi_{0}$ being a constant of integration and where for the sake of simplicity we have introduced the notations:

$$
A_{\varepsilon_{\varphi}}(E)=2 \arctan \left[\left(\frac{1+\varepsilon}{1-\varepsilon}\right)^{\frac{1}{2}} \tan \frac{E}{2}\right] \text {, }
$$

and

$$
K=\frac{H}{n \tilde{a}^{2}\left(1-\varepsilon_{\varphi}^{2}\right)^{\frac{1}{2}}} .
$$

From Eq. (3.22) and (3.19) and the definition of $\varepsilon_{\varphi}=2 \tilde{\varepsilon}-\varepsilon_{t}$ we have:

$$
\varepsilon_{\varphi}=\varepsilon_{t}\left(1+\frac{A D}{B C_{0}}-\frac{A I}{B H}\right)=\varepsilon_{r}\left(1+\frac{A D}{2 B C_{0}}-\frac{A I}{B H}\right)
$$

then, as shown by straightforward calculations:

$$
\begin{aligned}
& \varepsilon_{\varphi}=\varepsilon_{r}\left(1+\frac{G \mu}{2 a_{r} c^{2}}\right)= \\
& \left\{1+\frac{2 E}{G^{2} M^{2}}\left[1+\left(\frac{1}{2} v-\frac{15}{2}\right) \frac{E}{c^{2}}\right]\left[J^{2}-6 \frac{G^{2} M^{2}}{c^{2}}\right]\right\}^{\frac{1}{2}},
\end{aligned}
$$

and

$$
K=\frac{J}{\left(J^{2}-\frac{6 G^{2} M^{2}}{c^{2}}\right)^{\frac{1}{2}}} .
$$

As it is clear from Eqs. (3.20) and (3.21), the radial variable $r$ reaches its successive minima "periastron passages" for $E=0,2 \pi, 4 \pi, \ldots$ The periastron therefore precesses at each turn by the angle $\Delta \varphi=2 \pi(L-1)$, which if $J \gg \frac{G M}{c}$ reduces to the well-known result:

$$
\Delta \phi=6 \pi \frac{G^{2} M^{2}}{J^{2} c^{2}}+\mathrm{O}\left(\frac{1}{c^{4}}\right)=\frac{6 \pi G M}{a_{R}\left(1-\varepsilon_{r}\right) c^{2}}+\mathrm{O}\left(\frac{1}{c^{4}}\right) .
$$

Contrarily to the usual approach which derives first the orbit by eliminating the time between Eq. (3.12) and (3.13) before working out the motion on the orbit we find the orbit by eliminating $E$ between Eq. (3.18) and (3.23)-(3.25). With the aim to simplify the formulae we introduce the notation $f$ for the polar angle counted from a periastron and corrected for the periastron precession i.e.:

$$
f=\frac{\phi-\phi_{0}}{K}
$$

We must eliminate $E$ between:

$$
r=a_{r}\left(1-\varepsilon_{r} \cos E\right),
$$

and

$$
f=A_{\varepsilon_{\varphi}}(E) .
$$

In order to get, it is convenient to play a new conchoidal transformation on $r$ writing:

$$
r=\frac{\varepsilon_{r}}{\varepsilon_{\varphi}} a_{r}\left(1-\varepsilon_{\varphi} \cos E\right)+a_{r}\left(1-\frac{\varepsilon_{r}}{\varepsilon_{\varphi}}\right) .
$$

From the definition of $A_{\varepsilon_{\varphi}}(E)$ we have:

$$
1-\varepsilon_{\varphi} \cos E=\frac{1-\varepsilon_{\varphi}^{2}}{1+e_{\varphi} A_{\varepsilon_{\varphi}}(E)}=\frac{1-\varepsilon_{\varphi}^{2}}{1+\varepsilon_{\varphi} \cos f} .
$$

Moreover we find from Eq. (3.26) that the radial displacement appearing in Eq. (3.28) is simply

$$
a_{r}\left(1-\frac{\varepsilon_{r}}{\varepsilon_{\varphi}}\right)=\frac{G \mu}{2 c^{2}}
$$

so that we find the polar equation of the relative orbit as:

$$
r=\left(a_{r}-\frac{G \mu}{2 c^{2}}\right) \frac{1-\varepsilon_{\varphi}^{2}}{1+\varepsilon_{\varphi} \cos f}+\frac{G \mu}{2 c^{2}}
$$

This equations means that the relative orbit is the conchoid of a precessing ellipse, which means that it is obtained from an ellipse: $r^{\prime}=l\left(1+e \cos \phi^{\prime}\right)^{-1}$ by a radial displacemnet $r=r^{\prime}+$ const together with an angular homothetic transformation: $\phi=\operatorname{const} \cdot \phi^{\prime}$. Let us finally note that the relative orbit con also be written as:

$$
r=\frac{a_{r}\left(1-\varepsilon_{r}^{2}\right)}{1+\varepsilon_{r} \cos f^{\prime}},
$$

with

$$
f^{\prime}=f+2\left(\frac{\varepsilon_{2 f}}{\varepsilon_{r}}\right) \sin f .
$$

The conservation laws and the coordinate transformations which we have obtained here will reveal particularly useful to characterize the relativistic orbits, as we will see below.

\section{B. Relativistic Quasi-Elliptical Orbits}

The relativistic motions of each body are obtained by replacing the solutions for the relative motion, $t(E), r(E), \varphi(E)$, in the $\mathrm{PN}$ center of mass formulae Eq. 
(3.4) (see [54]). We see first that the polar angle of the first object (of mass $m_{1}$ ) is the same as the relative polar angle and that the polar angle of the second object (mass $m_{2}$ ) is simply $\varphi+\pi$. Therefore it is sufficient to work out the radial motions. From Eq. (3.4) we have by replacing $V^{2}$ in the relativistic corrections with $\frac{2 G M}{R}+2 E \simeq \frac{2 G M}{R}-\frac{G M}{a_{r}}$ :

$$
r=\frac{m_{2} R}{M}+\frac{G \mu\left(m_{1}-m_{2}\right)}{2 m c^{2}}\left(1-\frac{R}{a_{R}}\right)
$$

(and similar results for the second object by exchanging $m_{1}$ and $m_{2}$ ) which shows remarkably enough, that $r$ can also be written in a quasi-Newtonian form:

$$
r=a_{r^{\prime}}\left(1-\varepsilon_{r^{\prime}} \cos E\right),
$$

with

$$
\begin{aligned}
& a_{r^{\prime}}=\frac{m_{2}}{M} a_{r}, \\
& \varepsilon_{r^{\prime}}=e_{R}\left[1-\frac{G m_{1}\left(m_{1}-m_{2}\right.}{2 M a_{r} c^{2}}\right],
\end{aligned}
$$

and where as before:

$$
\begin{aligned}
& n\left(t-t_{0}\right)=E-\varepsilon_{t} \sin E, \\
& \phi-\phi_{0}=K A_{\varepsilon_{\phi}}(E) .
\end{aligned}
$$

The orbit in space of the first object can be written by using the same method as in the preceding Section for the relative orbit, that is:

$$
r=\frac{\varepsilon_{r^{\prime}}}{\varepsilon_{\varphi}} a_{r^{\prime}}\left(1-\varepsilon_{\varphi} \cos E\right)+a_{r^{\prime}}\left(1-\frac{\varepsilon_{r^{\prime}}}{\varepsilon_{\varphi}}\right) .
$$

One finds:

$$
a_{r^{\prime}}\left(1-\frac{\varepsilon_{r^{\prime}}}{\varepsilon-\phi}\right)=\frac{G m_{1}^{2} m_{2}}{2 M^{2} c^{2}},
$$

hence we find that the orbits is conchoid of a precessing ellipse with

$$
r^{\prime}=\left(a_{r^{\prime}}-\frac{G m_{1}^{2} m_{2}}{2 M^{2} c^{2}}\right) \frac{1-\varepsilon_{\phi}^{2}}{1+\varepsilon_{\phi} \cos \left(\frac{\phi-\phi_{0}}{L}\right)}+\frac{G m_{1}^{2} m_{2}}{2 M^{2} c^{2}}
$$

Summarizing then gathering our results for the ellipticlike $(E<0)$ PN motion in the PN center of mass frame, we have:

$$
\begin{gathered}
n\left(t-t_{0}\right)=E-\varepsilon_{t} \sin E \\
\phi-\phi_{0}=K 2 \arctan \left[\left(\frac{1+\varepsilon_{\phi}}{1-\varepsilon_{\phi}}\right)^{\frac{1}{2}} \tan \frac{E}{2}\right],
\end{gathered}
$$

$$
\begin{aligned}
& r=a_{r}\left(1-\varepsilon_{r} \cos E\right), \\
& r^{\prime}=a_{r^{\prime}}\left(1-e_{r^{\prime}} \cos E\right),
\end{aligned}
$$

with

$$
\begin{aligned}
& a_{r}=\frac{G M}{2 E}\left[1-\frac{1}{2}(v-7) \frac{E}{c^{2}}\right], \\
& n=\frac{(-2 E)^{\frac{3}{2}}}{G M}\left[1-\frac{1}{4}(v-15) \frac{E}{c^{2}}\right] .
\end{aligned}
$$

and $K, \varepsilon_{t}, e_{\varphi}, \varepsilon_{r}, e_{r}, a_{r}, e_{r^{\prime}}, a_{r^{\prime}}$ given in terms of the total angular momentum by unit reduced mass in the center of mass frame, $E$ and $J$, by Eq. (3.19), (3.27), (3.26), and interchange of $m_{1}$ and $m_{2}$ for $\varepsilon_{r^{\prime}}, a_{r^{\prime}}$. The above equations are very similar to the standard Newtonian solutions of the non-relativistic two-body problem.

\section{Relativistic Quasi-Hyperbolic Orbits}

The simplest method to obtain the Post-Newtonian motion in the hyperbolic-like case $(E>0)$ consists simply in making, in Eqs. (3.29)-(3.30), the analytic continuation from $E<0$ to $E>0$, passing below $E=0$ in the complex $E$ plane and replacing the parameter $E$ by $i F\left(i^{2}=-1\right)$. The proof that this yields to a correct parametric solution consists in noticing, on one hand, that $K$ and the various eccentricities are analytic in $E$, near $E=0$, and that if one replaces the parametric solution (3.29)-(3.30) and the corresponding expressions of $\varepsilon_{t}, e_{\varphi}, \varepsilon_{r}, e_{r}, a_{r}, e_{r^{\prime}}, a_{r^{\prime}}$ in terms of $E$ and $J$ in $\left(\frac{d r}{d t}\right)^{2}$ and in $\left(\frac{d \phi}{d t}\right)^{2}$, one finds that Eq. (3.12) and the square of Eq. (3.13) are satisfied identically, modulo $O\left(\frac{1}{c^{4}}\right)$, and that the resulting identities are analytic in $E$ and $E$ as purely imaginary ones. One finds:

$\bar{n}\left(t-t_{0}\right)=\varepsilon_{t} \sinh F-F$,

$$
\begin{aligned}
& \phi-\phi_{0}=K 2 \arctan \left[\left(\frac{\varepsilon_{\phi}+1}{\varepsilon_{\phi}-1}\right)^{\frac{1}{2}} \tanh \frac{F}{2}\right], \\
& r=\bar{a}_{r}\left(1-\varepsilon_{r} \cos F\right), \\
& r^{\prime}=\bar{a}_{r^{\prime}}\left(1-\varepsilon_{r^{\prime}} \cos F\right),
\end{aligned}
$$

where $K, \varepsilon_{t}, \varepsilon_{\phi}, \varepsilon_{r}, \varepsilon_{r^{\prime}}$ are functions of $E$ and $J$, as before, but where it has been conveninet to introduce the opposites of analytic continuations of the semi-major axes:

$$
\bar{a}_{r^{\prime}}=\frac{G M}{2 E}\left[1-\frac{1}{2}(v-7) \frac{E}{c^{2}}\right],
$$


and $\bar{a}_{r^{\prime}}=\frac{m_{1} \bar{a}_{r}}{M}$ and the modulus of the analytic continuation of the mean motion:

$$
\bar{n}=\frac{(2 M)^{\frac{3}{2}}}{G M}\left[1-\frac{1}{4}(v-15) \frac{E}{c^{2}}\right] .
$$

\section{Relativistic Quasi-Parabolic Orbits}

The quasi-parabolic post-Newtonian motion ( $E=0)$ can be obtained as a limit when $E \rightarrow 0$. For istance, let us start from the quasi-elliptic solution in Eq. (3.29) and pose

$$
E=\left(\frac{-2 E}{G^{2} M^{2}}\right)^{\frac{1}{2}} x
$$

Taxing now the limit $E \rightarrow 0^{-}$, holding $x$ fixed, yields the following parametric representation of the quasi parabolic motion:

$$
\begin{gathered}
t-t_{0}=\frac{1}{2 G^{2} M^{2}}\left[\left(J^{2}+(2-2 v) \frac{G^{2} M^{2}}{c^{2}} x+\frac{1}{3} x^{3}\right)\right], \\
\phi-\phi_{0}=\frac{J}{\left.\left(J^{2}-6\right) \frac{G^{2} M^{2}}{c^{2}}\right)^{\frac{1}{2}}} 2 \arctan \frac{x}{\left(J^{2}-\frac{6 G^{2} M^{2}}{c^{2}}\right)^{\frac{1}{2}}} \\
r=\frac{1}{2 G M}\left[\left(J^{2}+(v-6) \frac{G^{2} M^{2}}{c^{2}} x+x\right)\right] .
\end{gathered}
$$

Moreover let us point out that our solutions (for the three cases $E<0, E>0$ and $E=0$ ) have been written in a suitable form when $J^{2}>6\left(\frac{G M}{c}\right)^{2}$. However the validity of our solutions can be extended to the range $J^{2} \leq 6\left(\frac{G M}{c}\right)^{2}$ by first replacing in the solutions for the angular motion, the second equation of (3.29) and (3.31), and considering the Eqs. (3.32)-(3.34), the function arctan by cot (at the price of a simple modifiation of $\phi_{0}$ ) and then by making an analytic continuation in $J$. One ends up with an angular motion expressed by an argcoth which can also be approximatively replaced by its asymptotic behaviour for large arguments: $\operatorname{argcoth}(X) \sim \frac{1}{X}$. The case of purely radial motion $(J=0)$ is also obtained by taking the limit $J \rightarrow 0$ (at $E, F$ or respectively $x$ fixed). Finally a parametric representation of the general post-Newtonian motion in an arbitrary (post-Newtonian harmonic) coordinate system is obtained from our preceding center of mass solution by applying a general transformation of the Poincaré group $x^{\prime a}=L_{b}^{a} x^{b}+T^{a}[54]$.

\section{RELATIVISTIC ORBITS WITH GRAVITOMAGNETIC CORRECTIONS}

Using the orbital theory developed up to now for relativistic orbit, we have neglected terms of order $\frac{v^{3}}{c}$. However, we succeed in explaining, for instance, the perihelion precession of Mercury. In cases where $10^{-2} \leq \frac{v}{c} \leq 10^{-1}$, higher order terms like $\frac{v^{3}}{c}$ cannot be discarded in order to discuss consistently the problem of motion (see for example $[65,66])$. In this situations, we are dealing with gravitomagnetic corrections. Before discussing the theory of orbits under the gravitomagnetic effects, let us give some insight into gravitomagnetism and derive the corrected metric. Theoretical and experimental aspects of gravitomagnetism are discussed in [67, 68].

A remark is in order at this point: any theory combining, in a consistent way, Newtonian gravity together with Lorentz invariance has to include a gravitomagnetic field generated by the mass-energy currents. This is the case, of course, of GR: it was shown by Lense and Thirring [69-72], that a rotating mass generates a gravitomagnetic field, which, in turn, causes a precession of planetary orbits. In the framework of the linearized weak-field and slow-motion approximation of GR, the gravitomagnetic effects are induced by the off-diagonal components of the space-time metric tensor which are proportional to the components of the matter-energy current density of the source. It is possible to take into account two types of mass-energy currents. The former is induced by the matter source rotation around its center of mass: it generates the intrinsic gravitomagnetic field which is closely related to the angular momentum (spin) of the rotating body. The latter is due to the translational motion of the source: it is responsible of the extrinsic gravitomagnetic field [73]. Let us now discuss the gravitomagnetic effects in order to see how they affect the orbits.

\section{A. Gravitomagnetic Effects}

Starting from the Einstein field equations in the weak field approximation, one obtain the gravitoelectromagnetic equations and then the corrections on the metric ${ }^{4}$ [65]. The weak field approximation can be set as

$$
g_{\mu v}(x)=\eta_{\mu v}+h_{\mu \nu}(x), \quad\left|h_{\mu v}(x)\right| \ll<1,
$$

where $\eta_{\mu v}$ is the Minkowski metric tensor and $\left|h_{\mu v}(x)\right|<<1$ is a small deviation from it [39].

The stress-energy tensor for perfect - fluid matter is given by

$T^{\mu v}=\left(p+\rho c^{2}\right) u^{u} u^{v}-p g^{\mu v}$,

which, in the weak field approximation $p \ll \rho c^{2}$, is

${ }^{4}$ Notation: Latin indices run from 1 to 3 , while Greek indices run from 0 to 3 ; the flat space-time metric tensor is $\eta_{\mu v}=\operatorname{diag}(1,-1,-1,-1)$. 
$T^{00} \simeq \rho c^{2}, \quad T^{0 j} \simeq \rho c v^{j}, \quad T^{i j} \simeq \rho v^{i} v^{j}$.

From the Einstein field equations $G_{\mu v}=\left(8 \pi G / c^{4}\right) T_{\mu v}$, one finds

$$
\begin{aligned}
& \nabla^{2} h_{00}=\frac{8 \pi G}{c^{2}} \rho, \\
& \nabla^{2} h_{i j}=\frac{8 \pi G}{c^{2}} \delta_{i j} \rho, \\
& \nabla^{2} h_{0 j}=-\frac{16 \pi G}{c^{2}} \delta_{j l} \rho v^{l},
\end{aligned}
$$

where $\nabla^{2}$ is the standard Laplacian operator defined on the flat spacetime. To achieve Eqs. (4.2)-(4.4), the harmonic condition

$$
g^{\mu v} \Gamma_{\mu v}^{\alpha}=0
$$

has been used.

By integrating Eqs. (4.2)-(4.4), one obtains

$$
\begin{aligned}
& h_{00}=-\frac{2 \Phi}{c^{2}}, \\
& h_{i j}=-\frac{2 \Phi}{c^{2}} \delta_{i j}, \\
& h_{0 j}=\frac{4}{c^{3}} \delta_{j l} V^{l} .
\end{aligned}
$$

The metric is determined by the gravitational Newtonian potential

$$
\Phi(x)=-G \int \frac{\rho}{\left|\mathbf{x}-\mathbf{x}^{\prime}\right|} d^{3} x^{\prime},
$$

and by the vector potential $V^{l}$,

$$
V^{l}=-G \int \frac{\rho v^{l}}{\left|\mathbf{x}-\mathbf{x}^{\prime}\right|} d^{3} x^{\prime}
$$

given by the matter current density $\rho v^{l}$ of the moving bodies. This last potential gives rise to the gravitomagnetic corrections.

From Eqs. (4.1) and (4.5)-(4.9), the metric tensor in terms of Newton and gravitomagnetic potentials is

$$
\begin{aligned}
& d s^{2}=\left(1+\frac{2 \Phi}{c^{2}}\right) c^{2} d t^{2}-\frac{8 \delta_{l j} V^{l}}{c^{3}} c d t d x^{j}+ \\
& -\left(1-\frac{2 \Phi}{c^{2}}\right) \delta_{l j} d x^{i} d x^{j} .
\end{aligned}
$$

From Eq. (4.10) it is possible to construct a variational principle from which the geodesic equation follows. Then we can derive the orbital equations. As standard, we have

$$
\ddot{x}^{\alpha}+\Gamma_{\mu v}^{\alpha} \dot{x}^{\mu} \dot{x}^{v}=0
$$

where the dot indicates the differentiation with respect to the affine parameter. In order to put in evidence the gravitomagnetic contributions, let us explicitly calculate the Christoffel symbols at lower orders. By some straightforward calculations, one gets

$$
\begin{aligned}
& \Gamma_{00}^{0}=0 \\
& \Gamma_{0 j}^{0}=\frac{1}{c^{2}} \frac{\partial \Phi}{\partial x^{j}} \\
& \Gamma_{i j}^{0}=-\frac{2}{c^{3}}\left(\frac{\partial V^{i}}{\partial x^{j}}+\frac{\partial V^{j}}{\partial x^{i}}\right) \\
& \Gamma_{00}^{k}=\frac{1}{c^{2}} \frac{\partial \Phi}{\partial x^{k}} \\
& \Gamma_{0 j}^{k}=\frac{2}{c^{3}}\left(\frac{\partial V^{k}}{\partial x^{j}}-\frac{\partial V^{j}}{\partial x^{k}}\right) \\
& \Gamma_{i j}^{k}=-\frac{1}{c^{2}}\left(\frac{\partial \Phi}{\partial x^{j}} \delta_{i}^{k}+\frac{\partial \Phi}{\partial x^{i}} \delta_{j}^{k}-\frac{\partial \Phi}{\partial x^{k}} \delta_{i j}\right)
\end{aligned}
$$

In the approximation which we are going to consider, we are retaining terms up to the orders $\Phi / c^{2}$ and $V^{j} / c^{3}$. It is important to point out that we are discarding terms like $\left(\Phi / c^{4}\right) \partial \Phi / \partial x^{k}, \quad\left(V^{j} / c^{5}\right) \partial \Phi / \partial x^{k}, \quad\left(\Phi / c^{5}\right) \partial V^{k} / \partial x^{j}$, $\left(V^{k} / c^{6}\right) \partial V^{j} / \partial x^{i}$ and of higher orders. Our aim is to show that, in several cases like in tight binary stars, it is not correct to discard higher order terms in $v / c$ since physically interesting effects could come out. The geodesic equations up to $c^{-3}$ corrections are then

$$
\begin{aligned}
& c^{2} \frac{d^{2} t}{d \sigma^{2}}+\frac{2}{c^{2}} \frac{\partial \Phi}{\partial x^{j}} c \frac{d t}{d \sigma} \frac{d x^{j}}{d \sigma} \\
& -\frac{2}{c^{3}}\left(\delta_{i m} \frac{\partial V^{m}}{\partial x^{j}}+\delta_{j m} \frac{\partial V^{m}}{\partial x^{i}}\right) \frac{d x^{i}}{d \sigma} \frac{d x^{j}}{d \sigma}=0,
\end{aligned}
$$

for the time component, and

$$
\begin{aligned}
& \frac{d^{2} x^{k}}{d \sigma^{2}}+\frac{1}{c^{2}} \frac{\partial \Phi}{\partial x^{j}}\left(c \frac{d t}{d \sigma}\right)^{2}+\frac{1}{c^{2}} \frac{\partial \Phi}{\partial x^{k}} \delta_{i j} \frac{d x^{i}}{d \sigma} \frac{d x^{j}}{d \sigma} \\
& -\frac{2}{c^{2}} \frac{\partial \Phi}{\partial x^{l}} \frac{d x^{l}}{d \sigma} \frac{d x^{k}}{d \sigma}+\frac{4}{c^{3}}\left(\frac{\partial V^{k}}{\partial x^{j}}-\delta_{j m} \frac{\partial V^{m}}{\partial x^{k}}\right) c \frac{d t}{d \sigma} \frac{d x^{i}}{d \sigma}=0,
\end{aligned}
$$

for the spatial components.

In the case of a null-geodesic, it results $d s^{2}=d \sigma^{2}=0$. Eq. (4.10) gives, up to the order $c^{-3}$,

$$
c d t=\frac{4 V^{l}}{c^{3}} d x^{l}+\left(1-\frac{2 \Phi}{c^{2}}\right) d l_{\text {euclid }},
$$

where $d l_{\text {euclid }}^{2}=\delta_{i j} d x^{i} d x^{j}$ is the Euclidean length interval. Squaring Eq. (4.19) and keeping terms up to order $c^{-3}$, one finds 


$$
c^{2} d t^{2}=\left(1-\frac{4 \Phi}{c^{2}}\right) d l_{\text {euclid }}^{2}+\frac{8 V^{l}}{c^{3}} d x^{l} d l_{\text {euclid }} .
$$

Inserting Eq. (4.20) into Eq. (4.18), one gets, for the spatial components,

$$
\begin{aligned}
& \frac{d^{2} x^{k}}{d \sigma^{2}}+\frac{2}{c^{2}} \frac{\partial \Phi}{\partial x^{k}}\left(\frac{d l_{\text {euclid }}}{d \sigma}\right)^{2}-\frac{2}{c^{2}} \frac{\partial \Phi}{\partial x^{l}} \frac{d x^{l}}{d \sigma} \frac{d x^{k}}{d \sigma}+ \\
& \frac{4}{c^{3}}\left(\frac{\partial V^{k}}{\partial x^{j}}-\delta_{j m} \frac{\partial V^{m}}{\partial x^{k}}\right) \frac{d l_{\text {euclid }}}{d \sigma} \frac{d x^{j}}{d \sigma}=0 .
\end{aligned}
$$

Such an equation can be seen as a differential equation for $d x^{k} / d \sigma$ which is the tangent 3-vector to the trajectory. On the other hand, Eq. (4.21) can be expressed in terms of $l_{\text {euclid }}$ considered as a parameter. In fact, for null geodesics and taking into account the lowest order in $v / c, d \sigma$ is proportional to $d l_{\text {euclid }}$. From Eq. (4.17) multiplied for $\left(1+\frac{2}{c^{2}} \Phi\right)$, we have

$$
\frac{d}{d \sigma}\left(\frac{d t}{d \sigma}+\frac{2}{c^{2}} \Phi \frac{d t}{d \sigma}-\frac{4}{c^{4}} \delta_{i m} V^{m} \frac{d x^{i}}{d \sigma}\right)=0,
$$

and then

$$
\frac{d t}{d \sigma}\left(1+\frac{2}{c^{2}} \Phi\right)-\frac{4}{c^{4}} \delta_{i m} V^{m} \frac{d x^{i}}{d \sigma}=1
$$

where, as standard, we have defined the affine parameter so that the integration constant is equal to 1 [39]. Substituting Eq. (4.19) into Eq. (4.22), at lowest order in $v / c$, we find

$$
\frac{d l_{\text {euclid }}}{c d \sigma}=1 .
$$

In the weak field regime, the spatial 3-vector, tangent to a given trajectory, can be expressed as

$$
\frac{d x^{k}}{d \sigma}=\frac{c d x^{k}}{d l_{\text {euclid }}} .
$$

Through the definition

$$
e^{k}=\frac{d x^{k}}{d l_{\text {euclid }}}
$$

Eq. (4.21) becomes

$$
\begin{aligned}
& \frac{d e^{k}}{d l_{\text {euclid }}}+\frac{2}{c^{2}} \frac{\partial \Phi}{\partial x^{k}}-\frac{2}{c^{2}} \frac{\partial \Phi}{\partial x^{l}} e^{l} e^{k}+ \\
& +\frac{4}{c^{3}}\left(\frac{\partial V^{k}}{\partial x^{j}}-\delta_{j m} \frac{\partial V^{m}}{\partial x^{k}}\right) e^{j}=0,
\end{aligned}
$$

which can be expressed in a vector form as

$$
\frac{d \mathbf{e}}{d l_{\text {euclid }}}=-\frac{2}{c^{2}}[\nabla \Phi-\mathbf{e}(\mathbf{e} \cdot \nabla \Phi)]+\frac{4}{c^{3}}[\mathbf{e} \wedge(\nabla \wedge \mathbf{V})] .
$$

The gravitomagnetic term is the second one in Eq. (73) and it is usually discarded since considered not relevant. This is not true if $v / c$ is quite large as in the cases of tight binary systems or point masses approaching to black holes.

Our task is now to achieve explicitly the trajectories, in particular the orbits, corrected by such effects.

\section{B. Gravitomagnetically Corrected Orbits}

Orbits with gravitomagnetic effects can be obtained starting from the classical Newtonian theory and then correcting it by successive relativistic terms. Starting from the above considerations (see Sec. I, and III) we can see how gravitomagnetic corrections affect the problem of orbits. Essentially, they act as a further $v / c$ correction leading to take into account terms up to $c^{-3}$, as shown above. Let us start from the line element (4.10) which can be written in spherical coordinates. Here we assume the motion of pointlike bodies and then we can work in the simplified hypothesis $\Phi=-\frac{G M}{r}$ and $V^{l}=\Phi v^{l}$. It is

$$
\begin{aligned}
& d s^{2}=\left(1+\frac{2 \Phi}{c^{2}}\right) c d t^{2}-\left(1-\frac{2 \Phi}{c^{2}}\right) \\
& {\left[d r^{2}+r^{2} d \theta^{2}+r^{2} \sin ^{2} \theta d \varphi^{2}\right]} \\
& -\frac{8 \Phi}{c^{3}} c d t[\cos \theta+\sin \theta(\cos \varphi+\sin \varphi)] d r \\
& +\frac{8 \Phi}{c^{3}} c d t[\cos \theta(\cos \varphi+\sin \varphi)-\sin \theta] r d \theta \\
& +\frac{8 \Phi}{c^{3}} c d t[\sin \theta(\cos \varphi-\sin \varphi)] r d \varphi .
\end{aligned}
$$

As in the Newtonian and relativistic cases, from the line element (4.26), we can construct the Lagrangian

$$
\begin{aligned}
& \mathrm{L}=\left(1+\frac{2 \Phi}{c^{2}}\right) \dot{t}-\left(1-\frac{2 \Phi}{c^{2}}\right)\left[\dot{r}+r^{2} \dot{\theta}^{2}+r^{2} \sin ^{2} \theta \dot{\varphi}^{2}\right] \\
& -\frac{8 \Phi}{c^{3}} \dot{t}[\cos \theta+\sin \theta(\cos \varphi+\sin \varphi)] \dot{r} \\
& +\frac{8 \Phi}{c^{3}} \dot{t}[\cos \theta(\cos \varphi+\sin \varphi)-\sin \theta] r \dot{\theta} \\
& +\frac{8 \Phi}{c^{3}} \dot{t}[\sin \theta(\cos \varphi-\sin \varphi)] r \dot{\varphi} .
\end{aligned}
$$

Being, $L=1$, one can multiply both members for $\left(1+\frac{2 \Phi}{c^{2}}\right)$. In the planar motion condition $\theta=\pi / 2$, we obtain

$$
E^{2}-\left(1+\frac{2 \Phi}{c^{2}}\right)\left(1-\frac{2 \Phi}{c^{2}}\right)\left(\dot{r}^{2}+\frac{L^{2}}{r^{2}}\right)
$$




$$
\begin{aligned}
& -\frac{8 \Phi E}{c^{3}}[(\cos \phi+\sin \phi) \dot{r}-(\cos \phi-\sin \phi) \dot{\phi}] \\
& =\left(1+\frac{2 \Phi}{c^{2}}\right),
\end{aligned}
$$

and then, being $\frac{2 \Phi}{c^{2}}=-\frac{R_{s}}{r}$ (where $R_{s}$ is the Schwarzschild radius) and $u=\frac{1}{r}$ it is

$$
\begin{aligned}
& E^{2}-h^{2}\left(1-R_{s}^{2} u^{2}\right)\left(u^{\prime 2}+u^{2}\right)+ \\
& \frac{4 R_{S} u E}{c}\left[(\cos \phi+\sin \phi) u^{\prime}+(\cos \phi-\sin \phi) u^{2}\right] \\
& =\left(1-R_{S} u\right) .
\end{aligned}
$$

By deriving such an equation, it is easy to show that, if the relativistic and gravitomagnetic terms are discarded, the Newtonian theory is recovered, being

$$
u^{\prime \prime}+u=\frac{R_{s}}{2 L^{2}} .
$$

This result probes the self-consistency of the problem. However, it is nothing else but a particular case since we have assumed the planar motion. This planarity condition does not hold in general if gravitomagnetic corrections are taken into account. >From the above Lagrangian (4.26), it is straightforward to derive the equations of motion

$$
\begin{aligned}
& \ddot{r}=\frac{1}{c r\left(r c^{2}+2 G M\right)}\left[c\left(r c^{2}+G M\right)\left(\dot{\theta}^{2}+\sin ^{2} \theta \dot{\phi}^{2}\right) r^{2}+\right. \\
& \mid-4 G M \dot{t}((\cos \theta(\cos \phi+\sin \phi)-\sin \theta) \dot{\theta}+ \\
& \left.\quad \sin \theta(\cos \phi-\sin \phi) \dot{\phi}) r+c G M \dot{r}^{2}-c G M \dot{t}^{2}\right], \\
& \ddot{\phi}=-\frac{2}{r^{2}\left(r c^{3}+2 G M c\right)} c \cot \theta\left(r c^{2}+2 G M\right) \dot{\theta} \dot{\phi} r^{2} \\
& +\dot{r}\left(2 G M \csc \theta(\sin \phi-\cos \phi) \dot{t}+c r\left(r c^{2}+G M\right) \dot{\phi}\right), \\
& \ddot{\theta}=\frac{1}{r^{2}\left(r c^{3}+2 G M c\right)} c \cos \theta r^{2}\left(r c^{2}+2 G M\right) \sin \theta \dot{\phi}^{2} \\
& +\dot{r}(4 G M(\cos \theta(\cos \varphi+\sin \varphi)-\sin \theta) \dot{t}+ \\
& \left.-2 c r\left(r c^{2}+G M\right) \dot{\theta}\right)
\end{aligned}
$$

corresponding to the spatial components of the geodesic Eq. (4.21). The time component $\ddot{t}$ is not necessary for the discussion of orbital motion. Being the Lagrangian (4.26) $\mathrm{L}=1$ it is easy to achieve a first integral for $\dot{r}$ which is a natural constrain equation related to the energy.
Our aim is to show how gravitomagnetic effects modify the orbital motion and what the parameters determining the stability of the problem are. As we will see, the energy and the mass, essentially, determine the stability. Beside the standard periastron precession of GR, a nutation effect is genuinely induced by gravitomagnetism and stability greatly depends on it. A fundamental issue for this study is to achieve the orbital phase space portrait.

In Fig. (2), the results for a given value of nutation angular velocity with a time span of 10000 steps is shown. It is interesting to see that, by increasing the initial nutation angular velocity, being fixed all the other initial conditions, we get curves with decreasing frequencies for $\dot{r}(t)$ and $\ddot{r}(t)$. This fact is relevant to have an insight on the orbital motion stability (see Fig. 7). The effect of gravitomagnetic terms are taken into account, in Fig. (3-4), showing the basic orbits (left) and the orbit with the associated velocity field in false colors (right). From a rapid inspection of the right panel, it is clear the sudden changes of velocity direction induced by the gravitomagnetic effects.

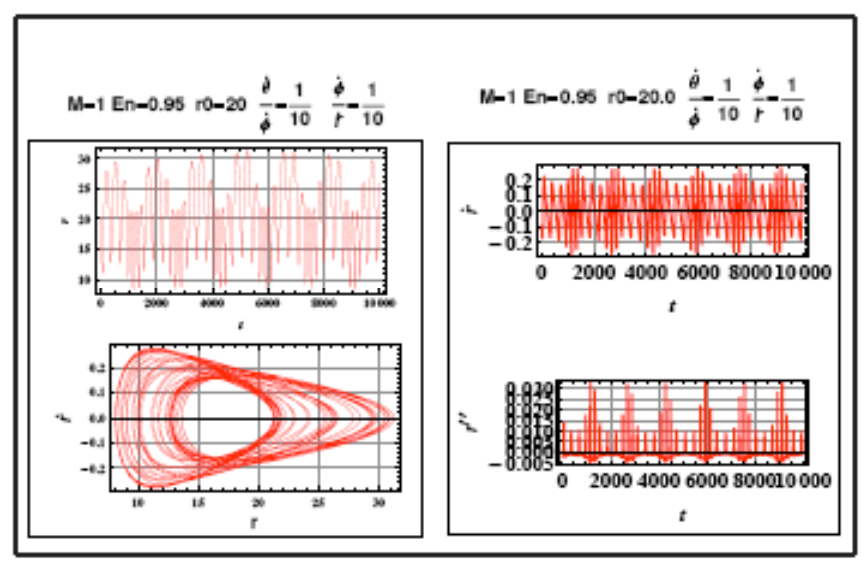

Fig (2). Plots along the panel lines of: $r(t)$ (upper left),phase portrait of $r(t)$ versus $\dot{r}(t)$ (bottom left), $\dot{r}(t)$ (upper right) and $\ddot{r}(t)$ (bottom right) for a star of $1 M_{\odot}$. The examples we are showing were obtained solving the system for the following parameters and initial conditions: $\mu \approx 1 M_{\odot}, E=0.95, \phi_{0}=0$, $\theta_{0}=\frac{\pi}{2}, \dot{\theta}_{0}=\frac{1}{10} \dot{\phi}_{0}, \dot{\phi}_{0}=-\frac{1}{10} \dot{r}_{0}$ and $\dot{r}_{0}=-\frac{1}{100}$ and $r_{0}=20 \mu$. The stiffness is evident from the trend of $\dot{r}(t)$ and $\ddot{r}(t)$.

To show the orbital velocity field, a rotation and a projection of the orbits along the axes of maximal energy can be performed. In other words, by a Singular Value Decomposition of the de-trended positions and velocities, the eigenvectors corresponding to the largest eigenvalues can be selected, and, of course, those representing the highest energy components (see Figs. 3-4).

The above differential equations for the parametric orbital motion are non-linear and with time-varying coefficients. In order to have a well-posed Cauchy problem, we have to define:

- the initial and final boundary condition problems;

- the stability and the dynamical equilibrium of solutions. 


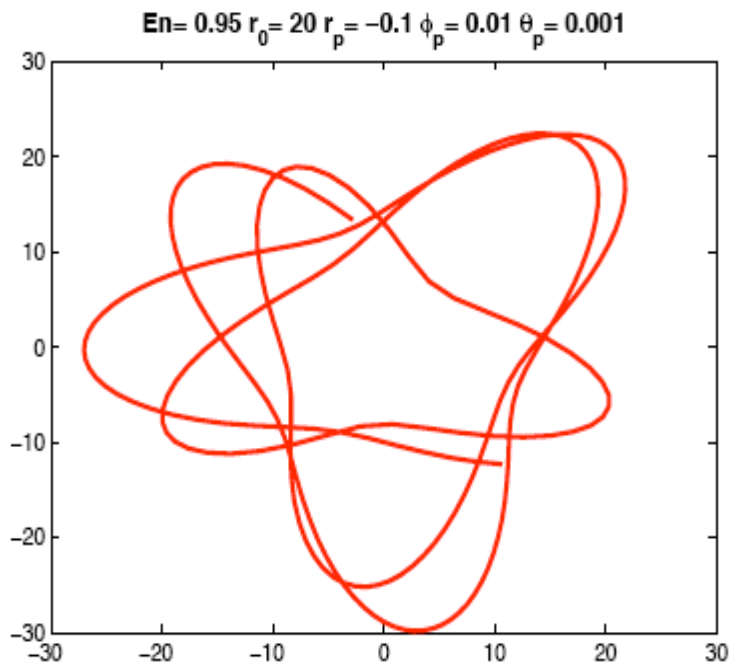

Fig. (3). Plots of basic orbits (left) The initial values are: ; $E_{n}=0.95$ in mass units; $r_{0}=20$ in Schwarzschild radii; $\dot{\phi}=-\frac{\dot{r}}{10} ;$ $\dot{\theta}=\frac{\dot{\phi}}{10}$.

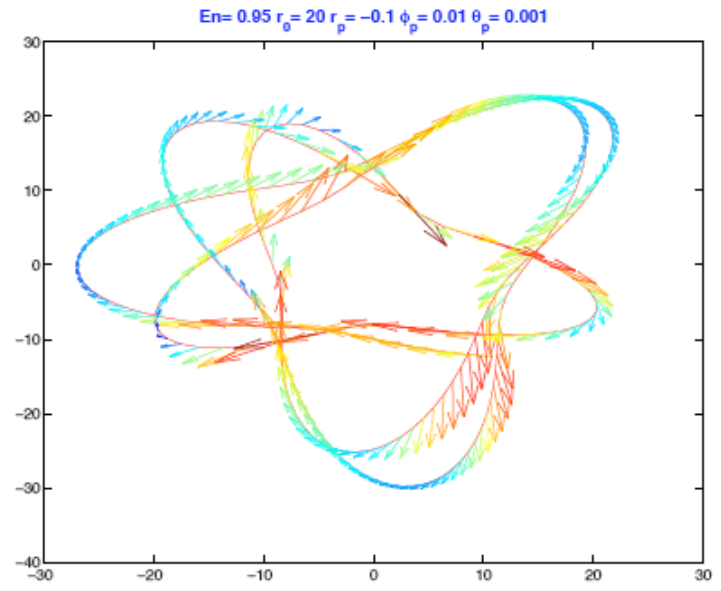

Fig. (4). Plots of basic orbits with the associated velocity field. The arrows indicate the instantaneous velocities. The initial values are: ; $E_{n}=0.95$ in mass units; $r_{0}=20$ in Schwarzschild radii; $\dot{\phi}=-\frac{\dot{r}}{10} ;$ $\dot{\theta}=\frac{\dot{\phi}}{10}$.

We can start by solving the Cauchy problem, as in the classical case, for the initial condition putting $\dot{r}=0, \dot{\phi}=0$, $\dot{\theta}=0$ and $\theta=\frac{\pi}{2}$ and the result we get is that the orbit is not planar being $\ddot{\theta} \neq 0$. In this case, we are compelled to solve numerically the system of second order differential equations and to treat carefully the initial conditions, taking into account the high non-linearity of the system. A similar discussion, but for different problems, can be found in [74, 75].

A series of numerical trials on the orbital parameters can be done in order to get an empirical insight on the orbit stability. The parameters involved in this analysis are the

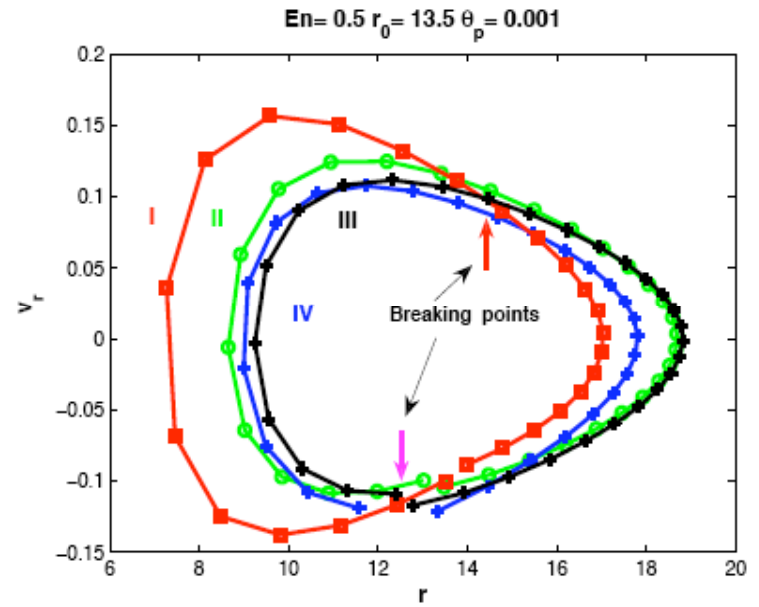

Fig. (5). Breaking points examples: on the left panel, the first four orbits in the phase plane are shown: the red one is labelled I, the green is II, the black is III and the fourth is IV. As it is possible to see, the orbits in the phase plane are not closed and they do not overlap at the orbital closure points; we have called this feature breaking points. In this dynamical situation, a small perturbation can lead the system to a transition to the chaos as prescribed by the Kolmogorov-Arnold-Moser (KAM) theorem [25]. On the right panel, it is shown the initial orbit with the initial (square) and final (circles) points marked in black.

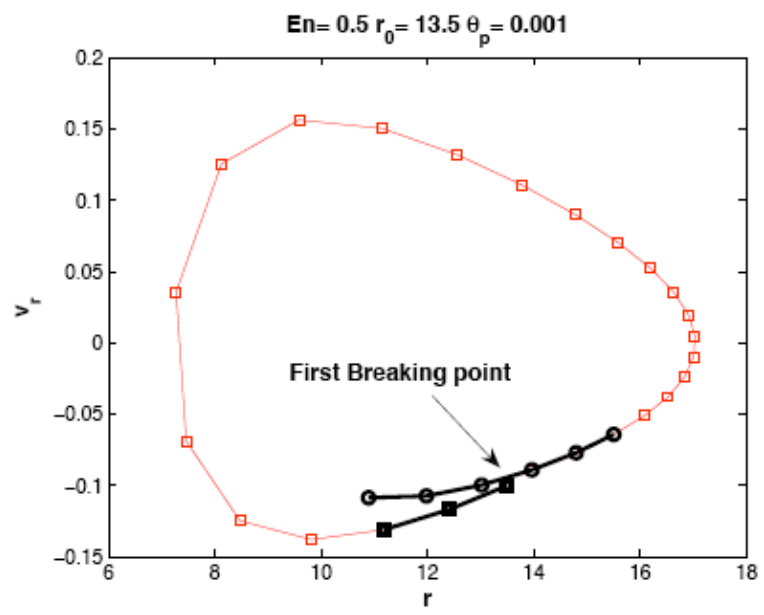

Fig. (6). In this figure it is shown the initial orbit with the initial (squares) and final(circles) points marked in black.

mass, the energy, the orbital radius, the initial values of $r, \phi, \theta$ and the angular precession and nutation velocities $\dot{\varphi}$ and $\dot{\theta}$ respectively. We have empirically assumed initial conditions on $\dot{r}, \phi$ and $\dot{\theta}$.

The trials can be organized in two series, i.e. constant mass and energy variation and constant energy and mass variation.

- In the first class of trials, we assume the mass equal to $M=$

$1 M$ and the energy $E_{n}$ (in mass units) varying step by

step. The initial orbital radius $r_{0}$ can be changed, according to the step in energy: this allows to find out numerically the dynamical equilibrium of the orbit. We have also chosen, as varying parameters, the ratios of the 


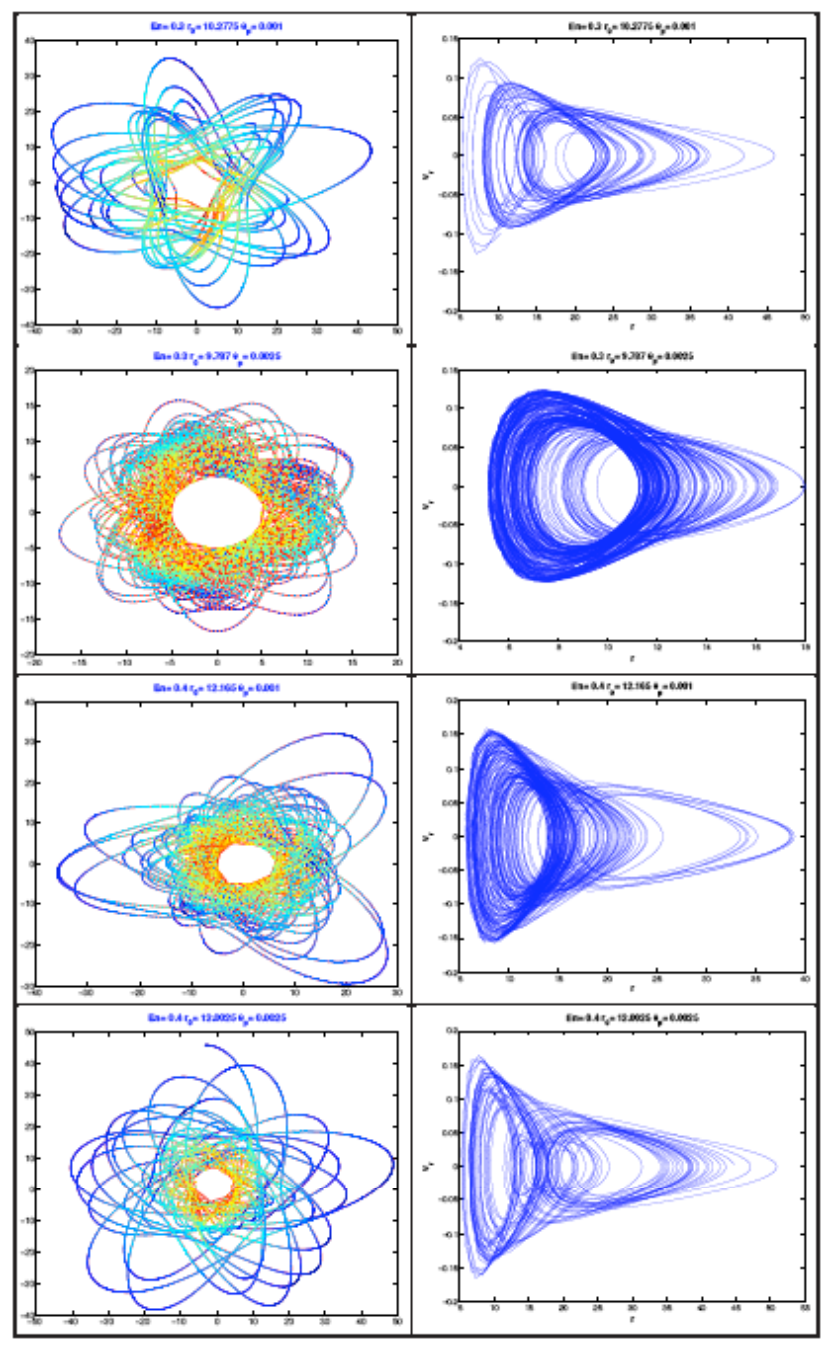

Fig (7). Plots of orbits with various energy values. For each value of energy, four plots are shown: the first on the left column is the orbit, with the orbital velocity field in false colors. The color scale goes from blue to red in increasing velocity. The second on the left column is the orbit with a different nutation angular velocity. On the right column, the phase portraits $\dot{r}=\dot{r}(r(t))$ are shown. Energy varies from 0.3 to 0.4 , in mass units. The stability of the system is highly sensitive either to very small variation of $r_{0}$ or to variation on the initial conditions on both precession and nutation angular velocities: it is sufficient a variation of few percent on $r_{0}$ to induce system instability.

precession angular velocity $\dot{\phi}$ to the radial angular velocity $\dot{r}$ and the ratio of the nutation angular velocity $\dot{\theta}$ and the precession angular velocity $\dot{\phi}$. The initial condition on $\varphi$ has been assumed to be $\phi_{0}=0$ and the initial condition on $\theta$ has been $\theta_{0}=\frac{\pi}{2}$. For $M=1$ (in Solar masses), $\frac{\dot{\theta}}{\dot{\phi}}=\frac{1}{2}$ and $\dot{\varphi}=-\frac{\dot{r}}{10}$, can be found out two different empirical linear equations, according to the different values of $\dot{\theta}, \dot{\phi}$. One obtains a rough guess of the initial distance $r_{0}=r_{0}\left(E_{n}\right)$ around which it is possible to find a guess on the equilibrium of the initial radius, followed by trials and errors procedure.

- In the second class of trials, we have assumed the variation of the initial orbital radius for different values of mass at a constant energy value equal to $E_{n}=0.95$ in mass units. With this conditions, we assume $\dot{\varphi}=\frac{\dot{r}}{10}$ and assume that $\dot{\theta}$ takes the two values $1 / 2$ and $1 / 10$. One can approach the problem also considering the mass parameterization, at a given fixed energy, to have an insight of the effect of mass variation on the initial conditions. The masses have been varied between 0.5 and 20 Solar masses and the distances have been found to vary according to the two 3rd-order polynomial functions, according to the different values of $\dot{\theta}$ with respect to the mass (for details see [65]).

In summary, the numerical calculations, if optimized, allow to put in evidence the specific contributions of gravitomagnetic corrections on orbital motion. In particular, specific contributions due to nutation and precession emerge when higher order terms in $v / c$ are considered.

The conclusion of this part of the review is that orbits are highly characterized by the velocity regime of the moving bodies. The order of the parameter $v / c$ determines the global shape of the trajectories. Our task is now to show how the motion of sources is related to the features of emitted GWs, in particular to their production and to the profile of waves.

\section{PART II}

\section{Production and Signature of Gravitational Waves}

The first derivation of gravitational radiation in GR is due to Einstein. His initial calculation [76] was "marred by an error in calculation" (Einstein's words), and was corrected in 1918 [77] (albeit with an overall factor of two error). Modulo a somewhat convoluted history (discussed in great detail by Kennefick [78]) owing (largely) to the difficulties of analyzing radiation in a nonlinear theory, Einstein's final result stands today as the leading-order "quadrupole formula" for gravitational wave emission. This formula plays a role in gravity theory analogous to the dipole formula for electromagnetic radiation, showing GWs arise from accelerated masses exactly as electromagnetic waves arise from accelerated charges. The quadrupole formula tells us that GWs are difficult to produce - very large masses moving at relativistic speeds are needed. This follows from the weakness of the gravitational interaction. A consequence of this is that it is extremely unlikely there will ever be an interesting laboratory source of GWs. The only objects massive and relativistic enough to generate detectable GWs are astrophysical. Indeed, experimental confirmation of the existence of GWs has come from the study of binary neutron star systems - the variation of the mass quadrupole in such systems is large enough that GW emission changes the system's characteristics on a timescale short enough to be observed. Intuitively, it is clear that measuring these waves must be difficult - the weakness of the gravitational interaction ensures that the response of any detector to 
gravitational waves is very small. Nonetheless, technology has brought us to the point where detectors are now beginning to set interesting upper limits on GWs from some sources [79-82]. The first direct detection could be,hopefully, not too far in the future.

\section{GRAVITATIONAL WAVES IN LINEARIZED GRAVITY}

The most natural starting point for any discussion of GWs is linearized gravity [38, 43, 83]. Linearized gravity is an adequate approximation to GR when the spacetime metric, may be treated as deviating only slightly from a flat metric, $\eta_{\mu v}$ :

$$
g_{\mu v}=\eta_{\mu v}+h_{\mu v}, \quad\left\|h_{\mu v}\right\| \ll 1 \text {. Here }\left\|h_{\mu v}\right\| \text { means " the }
$$
magnitude of a typical non-zero component of $h_{\mu v} "$. Note that the condition $\left\|h_{u v}\right\| \ll 1$ requires both the gravitational field to be weak ${ }^{5}$, and in addition constrains the coordinate system to be approximately Cartesian. We will refer to $h_{\mu v}$ as the metric perturbation; as we will see, it encapsulates GWs, but contains additional, non-radiative degrees of freedom as well. In linearized gravity, the smallness of the perturbation means that we only keep terms which are linear in $h_{\mu v}$ - higher order terms are discarded. As a consequence, indices are raised and lowered using the flat metric $\eta_{\mu v}$. The metric perturbation $h_{\mu v}$ transforms as a tensor under Lorentz transformations, but not under general coordinate transformations.

We now compute all the quantities which are needed to describe linearized gravity. The components of the affine connection (Christoffel coefficients) are given by

$$
\begin{aligned}
& \Gamma_{v \rho}^{\mu}=\frac{1}{2} \eta^{\mu \sigma}\left(\partial_{\rho} h_{\rho v}+\partial_{v} h_{\sigma \rho}-\partial_{\sigma} h_{v \rho}\right) \\
& =\frac{1}{2}\left(\partial_{\rho} h^{\mu}{ }_{v}+\partial_{v} h_{\rho}^{\mu}-\partial^{\mu} h_{v \rho}\right) .
\end{aligned}
$$

Here $\partial_{\mu}$ means the partial derivative $\partial / \partial x^{\mu}$. Since we use $\eta_{\mu v}$ to raise and lower indices, spatial indices can be written either in the "up" position or the "down" position without changing the value of a quantity: $f^{x}=f_{x}$. Raising or lowering a time index, by contrast, switches sign: $f^{t}=-f_{t}$. The Riemann tensor we construct in linearized theory is then given by

$$
\begin{aligned}
& R_{v \rho \sigma}^{\mu}=\partial_{\rho} \Gamma_{v \sigma}^{\mu}-\partial_{\sigma} \Gamma_{v \rho}^{\mu} \\
& =\frac{1}{2}\left(\partial_{\rho} \partial_{v} h_{\sigma}^{\mu}+\partial_{\sigma} \partial^{\mu} h_{v \rho}-\partial_{\rho} \partial^{\mu} h_{v \sigma}-\partial_{\sigma} \partial_{v} h_{\rho}^{\mu}\right) .
\end{aligned}
$$

From this, we construct the Ricci tensor

$$
R_{\mu v}=R_{\mu \rho v}^{\rho}=\frac{1}{2}\left(\partial_{\rho} \partial_{v} h_{\mu}^{\rho}+\partial^{\rho} \partial_{\mu} h_{v \rho}-\square h_{\mu v}-\partial_{\mu} \partial_{v} h\right),
$$

${ }^{5}$ We will work in geometrized coordinates putting $c=G=1$ where $h=h_{\mu}^{\mu}$ is the trace of the metric perturbation, and $\square=\partial_{\rho} \partial^{\rho}=\nabla^{2}-\partial_{t}^{2}$ is the wave operator. Contracting once more, we find the curvature scalar:

$$
R=R_{\mu}^{\mu}=\left(\partial_{\rho} \partial^{\mu} h_{\mu}^{\rho}-\square h\right)
$$

and finally build the Einstein tensor:

$$
\begin{aligned}
& G_{\mu v}=R_{\mu v}-\frac{1}{2} \eta_{\mu v} R \\
& =\frac{1}{2}\left(\partial_{\rho} \partial_{v} h_{\mu}^{\rho}+\partial^{\rho} \partial_{\mu} h_{v \rho}-\square h_{\mu v}-\partial_{\mu} \partial_{\nu} h\right. \\
& \left.-\eta_{\mu \nu} \partial_{\rho} \partial^{\sigma} h^{\rho}{ }_{\sigma}+\eta_{\mu v} \square h\right) .
\end{aligned}
$$

This expression is a bit unwieldy. Somewhat remarkably, it can be cleaned up significantly by changing notation: rather than working with the metric perturbation $h_{\mu v}$, we use the trace-reversed perturbation $\bar{h}_{\mu v}=h_{\mu v}-\frac{1}{2} \eta_{\mu v} h$. (Notice that $\bar{h}_{\mu}^{\mu}=-h$, hence the name "trace reversed".) Replacing $h_{\mu v}$ with $\bar{h}_{\mu v}+\frac{1}{2} \eta_{\mu v} h$ in Eq. (5.2) and expanding, we find that all terms with the trace $h$ are canceled. What remains is

$$
G_{\mu \nu}=\frac{1}{2}\left(\partial_{\sigma} \partial_{v} \bar{h}_{\mu}^{\rho}+\partial^{\rho} \partial_{\mu} \bar{h}_{\mu \nu}-\square \bar{h}_{\mu \nu}-\eta_{\mu \nu} \partial_{\rho} \partial^{\sigma} \bar{h}_{\sigma}^{\rho}\right) .
$$

This expression can be simplified further by choosing an appropriate coordinate system, or gauge. Gauge transformations in general relativity are just coordinate transformations. A general infinitesimal coordinate transformation can be written as $x^{a 1}=x^{\mu}+\xi^{\mu}$, where $\xi^{\mu}\left(x^{v}\right)$ is an arbitrary infinitesimal vector field. This transformation changes the metric via

$$
h_{\mu v}{ }^{\prime}=h_{\mu v}-2 \partial_{(\mu} \xi_{v)},
$$

so that the trace-reversed metric becomes

$$
\begin{aligned}
& \bar{h}_{\mu \nu}{ }^{\prime}=h_{\mu \nu}{ }^{\prime}-\frac{1}{2} \eta_{\mu \nu} h^{\prime} \\
& =\bar{h}_{\mu \nu}-2 \partial_{(v} \xi_{\mu)}+\eta_{\mu \nu} \partial^{\rho} \xi_{\rho} .
\end{aligned}
$$

A class of gauges that are commonly used in studies of radiation are those satisfying the Lorenz gauge condition

$$
\partial^{\mu} \bar{h}_{\mu v}=0 \text {. }
$$

(Note the close analogy to Lorentz gauge ${ }^{6}$

\footnotetext{
${ }^{6}$ Fairly recently, it has become widely recognized that this gauge was in fact invented by Ludwig Lorenz, rather than by Hendrik Lorentz. The inclusion of the "t" seems most likely due to confusion between the similar names; see Ref. [84] for detailed discussion. Following the practice of Griffiths ([85], p. 421 ), we bow to the weight of historical usage in order to avoid any possible confusion.
} 
in electromagnetic theory, $\partial^{\mu} A_{\mu}=0$, where $A_{\mu}$ is the potential vector.) Suppose that our metric perturbation is not in Lorentz gauge. What properties must $\xi_{\mu}$ satisfy in order to impose Lorentz gauge? Our goal is to find a new metric $h_{\mu v}^{\prime}$ such that $\partial^{\mu} \bar{h}_{\mu v}^{\prime}=0$ :

$$
\begin{aligned}
& \partial^{\mu} \bar{h}_{\mu \nu}{ }^{\prime}=\partial^{\mu} \bar{h}_{\mu \nu}-\partial^{\mu} \partial_{v} \xi_{\mu}-\square \xi_{v}+\partial_{v} \partial^{\rho} \xi_{\rho} \\
& =\partial^{\mu} \bar{h}_{\mu \nu}-\square \xi_{v} .
\end{aligned}
$$

Any metric perturbation $h_{\mu v}$ can therefore be put into a Lorentz gauge by making an infinitesimal coordinate transformation that satisfies

$$
\square \xi_{v}=\partial^{\mu} \bar{h}_{\mu v} .
$$

One can always find solutions to the wave equation (5.5), thus achieving Lorentz gauge. The amount of gauge freedom has now been reduced

The amount of gauge freedom has now been reduced from 4 freely specifiable functions of 4 variables to 4 functions of 4 variables that satisfy the homogeneous wave equation $\square \xi^{v}=0$, or, equivalently, to 8 freely specifiable functions of 3 variables on an initial data hypersurface.

Applying the Lorentz gauge condition (5.4) to the expression (5.2) for the Einstein tensor, we find that all but one term vanishes:

$$
G_{\mu v}=-\frac{1}{2} \square \bar{h}_{\mu \nu}
$$

Thus, in Lorentz gauges, the Einstein tensor simply reduces to the wave operator acting on the trace reversed metric perturbation (up to a factor $-1 / 2$ ). The linearized Einstein equation is therefore

$$
\square \bar{h}_{\mu v}=-16 \pi T_{\mu v} ;
$$

in vacuum, this reduces to

$$
\square \bar{h}_{\mu v}=0 .
$$

Just as in electromagnetism, the equation (5.6) admits a class of homogeneous solutions which are superpositions of plane waves:

$$
\bar{h}_{\mu \nu}(x, t)=\operatorname{Re} \int d^{3} k A_{\mu \nu}(k) e^{i(k \cdot x-\omega t)} .
$$

Here, $\omega=|k|$. The complex coefficients $A_{\mu v}(k)$ depend on the wavevector $k$ but are independent of $x$ and $t$. They are subject to the constraint $k^{\mu} A_{\mu v}=0$ (which follows from the Lorentz gauge condition), with $k^{\mu}=(\omega, k)$, but are otherwise arbitrary. These solutions are the gravitational waves.

\section{A. Transverse Traceless (TT) Gauge in Globally Vacuum Spacetimes}

We now specialize to globally vacuum spacetimes in which $T_{\mu \nu}=0$ everywhere, and which are asymptotically flat (for our purposes, $h_{\mu v} \rightarrow 0$ as $r \rightarrow \infty$ ). Equivalently, we specialize to the space of homogeneous, asymptotically flat solutions of the linearized Einstein Eq. (5.6). For such spacetimes one can, along with choosing Lorentz gauge, further specialize the gauge to make the metric perturbation be purely spatial

$$
h_{00}=h_{0 i}=0
$$

and traceless

$$
h=h_{i}^{i}=0 \text {. }
$$

The Lorentz gauge condition (5.4) then implies that the spatial metric perturbation is transverse:

$$
\partial_{i} h_{i j}=0 \text {. }
$$

This is called the transverse-traceless gauge, or TT gauge. A metric perturbation that has been put into TT gauge will be written $h_{\mu v}^{\mathrm{TT}}$. Since it is traceless, there is no distinction between $h_{\mu \nu}^{\mathrm{TT}}$ and $\bar{h}_{\mu \nu}^{\mathrm{TT}}$.

The conditions (5.8) and (5.9) comprise 5 constraints on the metric, while the residual gauge freedom in Lorentz gauge is parameterized by 4 functions that satisfy the wave equation. It is nevertheless possible to satisfy these conditions, essentially because the metric perturbation satisfies the linearized vacuum Einstein equation. When the TT gauge conditions are satisfied, the gauge is completely fixed.

One might wonder why we would choose the TT gauge. It is certainly not necessary; however, it is extremely convenient, since the TT gauge conditions completely fix all the local gauge freedom. The metric perturbation $h_{\mu v}^{\mathrm{TT}}$ therefore contains only physical, non-gauge information about the radiation. In the TT gauge there is a close relation between the metric perturbation and the linearized Riemann tensor $R_{\mu \nu \rho \sigma}$ [which is invariant under the local gauge transformations (5.3) by Eq. (5.1)], namely

$$
R_{i 0 j 0}=-\frac{1}{2} \ddot{h}_{i j}^{\mathrm{TT}}
$$

In a globally vacuum spacetime, all non-zero components of the Riemann tensor can be obtained from $R_{i 0 j 0}$ via Riemann's symmetries and the Bianchi identity. In a more general spacetime, there will be components that are not related to radiation.

Transverse traceless gauge also exhibits the fact that gravitational waves have two polarization components. For example, consider a GW which propagates in the $z$ direction: $h_{i j}^{\mathrm{TT}}=h_{i j}^{\mathrm{TT}}(t-z)$ is a valid solution to the wave equation $\square h_{i j}^{\mathrm{TT}}=0$. The Lorentz condition $\partial_{z} h_{z j}^{\mathrm{TT}}=0$ implies that $h_{z j}^{\mathrm{TT}}(t-z)=$ constant . This constant must be zero to satisfy the condition that $h_{a b} \rightarrow 0$ as $r \rightarrow \infty$. The only non- 
zero components of $h_{i j}^{\mathrm{TT}}$ are then $h_{x x}^{\mathrm{TT}}, h_{x y}^{\mathrm{TT}}, h_{y x}^{\mathrm{TT}}$, and $h_{y y}^{\mathrm{TT}}$. Symmetry and the tracefree condition (5.9) further mandate that only two of these are independent:

$$
\begin{aligned}
& h_{x x}^{\mathrm{T} T}=-h_{y y}^{\mathrm{TT}} \equiv h_{+}(t-z) ; \\
& h_{x y}^{\mathrm{T} T}=h_{y x}^{\mathrm{T} T} \equiv h_{\times}(t-z) .
\end{aligned}
$$

The quantities $h_{+}$and $h_{\times}$are the two independent waveforms of the GW (see Fig. 8, 9) [38, 86].

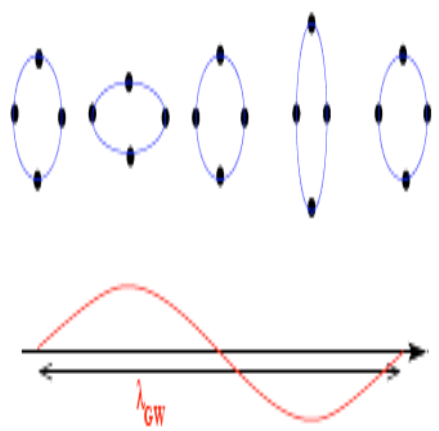

Fig. (8). We show how point particles along a ring move as a result of the interaction with a GW propagating in the direction perpendicular to the plane of the ring. This figure refers to a wave with + polarization.

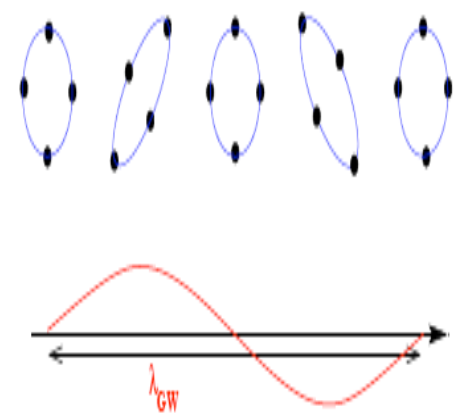

Fig. (9). We show how point particles along a ring move as a result of the interaction with a $\mathrm{GW}$ propagating in the direction perpendicular to the plane of the ring. This figure refers to a wave with $\times$ polarization

To illustrate the effect of GWs on free falling (FF) particles, we consider a ring of point particles initially at rest with respect to a FF frame attached to the center of the ring, as shown in Fig. $(\mathbf{8}, \mathbf{9})$.

\section{INTERACTION OF GRAVITATIONAL WAVES WITH A DETECTOR}

The usual notion of "gravitational force" disappears in GR, replaced instead by the idea that freely falling bodies follow geodesics in spacetime. Given a spacetime metric $g_{\mu v}$ and a set of spacetime coordinates $x^{\mu}$, geodesic trajectories are given by the equation

$$
\frac{d^{2} x^{\mu}}{d \tau^{2}}+\Gamma_{v \rho}^{\mu} \frac{d x^{v}}{d \tau} \frac{d x^{\rho}}{d \tau}=0,
$$

where $\tau$ is the proper time as measured by an observer travelling along the geodesic. By writing the derivatives in the above geodesic equation in terms of coordinate time $t$ rather than proper time $\tau$, and by combining the $\mu=t$ (i.e 0 coordinate) equation with the spatial, $\mu=j$ (i.e. spatial coordinates) equations, we obtain an equation for the coordinate acceleration:

$$
\begin{aligned}
& \frac{d^{2} x^{i}}{d t^{2}}=-\left(\Gamma_{00}^{i}+2 \Gamma_{0 j}^{i} v^{j}+\Gamma_{j k}^{i} v^{j} v^{k}\right)+ \\
& v^{i}\left(\Gamma_{00}^{0}+2 \Gamma_{0 j}^{0} v^{j}+\Gamma_{j k}^{0} v^{j} v^{k}\right),
\end{aligned}
$$

where $v^{i}=d x^{i} / d t$ is the coordinate.

Let us now specialize to linearized theory, with the nonflat part of our metric dominated by a GW in TT gauge. Further, let us specialize to non-relativistic motion for our test body. This implies that $v^{i} \ll 1$, and to a good approximation we can neglect the velocity dependent terms in Eq. (6.1):

$$
\frac{d^{2} x^{i}}{d t^{2}}+\Gamma_{00}^{i}=0 .
$$

In linearized theory and TT gauge,

$$
\Gamma_{00}^{i}=\Gamma_{i 0}^{0}=\frac{1}{2}\left(2 \partial_{t} h_{j 0}^{\mathrm{TT}}-\partial_{j} h_{00}^{\mathrm{TT}}\right)=0,
$$

since $h_{00}^{\mathrm{TT}}=0$. Hence, we find that $d^{2} x^{i} / d t^{2}=0$.

This result could mean that the GW has no effect.This is not true since it just tells us that, in TT gauge, the coordinate location of a slowly moving, freely falling (here in after FF) body is unaffected by the GWs. In essence, the coordinates move with the waves.

This result illustrates why, in GR, it is important to focus upon coordinate-invariant observables (a naive interpretation of the above result would be that freely falling bodies are not influenced by GWs). In fact the GWs cause the proper separation between two FF particles to oscillate, even if the coordinate separation is constant. Consider two spatial FF particles, located at $z=0$, and separated on the $x$ axis by a coordinate distance $L_{c}$. Consider a GW in TT gauge that propagates down the $z$ axis, $h_{u v}^{\mathrm{TT}}(t, z)$. The proper distance $L$ between the two particles in the presence of the GW is given by

$$
\begin{aligned}
& L=\int_{0}^{{ }^{c}} d x \sqrt{g_{x x}}=\int_{0}^{L^{c}} d x \sqrt{1+h_{x x}^{\mathrm{TT}}(t, z=0)} \\
& \simeq \int_{0}^{L^{c}} d x\left[1+\frac{1}{2} h_{x x}^{\mathrm{TT}}(t, z=0)\right]= \\
& =L_{c}\left[1+\frac{1}{2} h_{x x}^{\mathrm{TT}}(t, z=0)\right] .
\end{aligned}
$$

Notice that we use the fact that the coordinate location of each particle is fixed in TT gauge. In a gauge in which the 
particles move with respect to the coordinates, the limits of integration would have to vary. Eq. (6.2) tells us that the proper separation of the two particles oscillates with a fractional length change $\delta L / L$ given by

$$
\frac{\delta L}{L} \simeq \frac{1}{2} h_{x x}^{\mathrm{TT}}(t, z=0) .
$$

Although we used TT gauge to perform this calculation, the result is gauge independent; we will derive it in a different gauge momentarily. Notice that $h_{x x}^{\mathrm{TT}}$ acts as a strain, a fractional length change. The magnitude $h$ of a wave is often referred to as the "wave strain". The proper distance we have calculated here is a particularly important quantity since it directly relates to the accumulated phase which is measured by laser interferometric GW observatories . The "extra" phase $\delta \varphi$ accumulated by a photon that travels down and back the arm of a laser interferometer in the presence of a GW is $\delta \varphi=4 \pi \delta L / \lambda$, where $\lambda$ is the photon's wavelength and $\delta L$ is the distance the end mirror moves relative to the beam splitter ${ }^{7}$. We now give a different derivation of the fractional length change (6.3) based on the concept of geodesic deviation. Consider a geodesic in spacetime given by $x^{\mu}=z^{\mu}(\tau)$, where $\tau$ is the proper time, with four velocity $u^{\mu}(\tau)=d z^{\mu} / d \tau$. Suppose we have a nearby geodesic $x^{\mu}(\tau)=z^{\mu}(\tau)+L^{\mu}(\tau)$, where $L^{\mu}(\tau)$ is small. We can regard the coordinate displacement $L^{\mu}$ as a vector $\vec{L}=L^{\mu} \partial_{\mu}$ on the first geodesic; this is valid to first order in $\vec{L}$. Without loss of generality, we can make the connecting vector be purely spatial: $L^{\mu} u_{\mu}=0$. Spacetime curvature causes the separation vector to change with time, the geodesics will move further apart or closer together, with an acceleration given by the geodesic deviation equation

$$
u^{v} \nabla_{v}\left(u^{\rho} \nabla_{\rho} L^{\mu}\right)=-R_{v \rho \sigma}^{\mu}[\vec{z}(\tau)] u^{v} L^{\rho} u^{\sigma} ;
$$

see, e.g., Ref. [91]. This equation is valid to linear order in $L^{\mu}$; fractional corrections to this equation will scale as $L / L$, where $L$ is the lengthscale over which the curvature varies.

For application to GW detectors, the shortest lengthscale $L$ is the wavelength $\lambda$ of the GWs. Thus, the geodesic deviation equation will have fractional corrections of order $L / \lambda$. For ground-based detectors $L \lesssim$ a few $\mathrm{km}$, while $\lambda \gtrsim 3000 \mathrm{~km}$; thus the approximation will be valid. For

\footnotetext{
${ }^{7}$ This description of the phase shift only holds if $L \ll \lambda$, so that the metric perturbation does not change value very much during a light travel time. This condition will be violated in the high frequency regime for space-based GW detectors; a more careful analysis of the phase shift is needed in this case [87]. Furtheremore fractional corrections of order $L / \lambda$ can be important also for ground-based detectors. An important case regards relic GWs where the spectrum is continuous and in the high frequency portion of the band of ground-based detectors is involved. In this case, as it has been carefully explained in [88] fractional corrections of order L/lambda are important for ground-based detectors too. On the other hand, such fractional corrections of order $L / \lambda$ represent the so called "magnetic" component of the signal, as it has been carefully explained in [89].
}

detectors with $L \gtrsim \lambda$ (e.g. the space based detector LISA) the analysis here is not valid and other techniques must be used to analyze the detector.

A convenient coordinate system to analyze the geodesic deviation equation (6.4) is the local proper reference frame of the observer who travels along the first geodesic. This coordinate system is defined by the requirements

$$
z^{i}(\tau)=0, \quad g_{\mu v}(t, 0)=\eta_{\mu v}, \quad \Gamma_{v \rho}^{\mu}(t, 0)=0,
$$

which imply that the metric has the form

$$
d s^{2}=-d t^{2}+d x^{2}+O\left(\frac{x^{2}}{R^{2}}\right) .
$$

Here $R$ is the radius of curvature of spacetime, given by $R^{-2} \sim\left\|R_{\mu v \rho \sigma}\right\|$. It also follows from the gauge conditions (6.5) that proper time $\tau$ and coordinate time $t$ coincide along the first geodesic, that $\vec{u}=\partial_{t}$ and that $L^{\mu}=\left(0, L^{i}\right)$.

Consider now the proper distance between the two geodesics, which are located at $x^{i}=0$ and $x^{i}=L^{i}(t)$. From the metric (6.6) we see that this proper distance is just $|L|=\sqrt{L_{i} L_{i}}$, up to fractional corrections of order $L^{2} / R^{2}$. For a GW of amplitude $h$ and wavelength $\lambda$ we have $R^{-2} \sim h / \lambda^{2}$, so the fractional errors are $\sim h L^{2} / \lambda^{2}$. (Notice that $R \sim L / \sqrt{h}$ the wave's curvature scale $R$ is much larger than the lengthscale $L$ characterizing its variations.) Since we are restricting attention to detectors with $L \ll \lambda$, these fractional errors are much smaller than the fractional distance changes $\sim h$ caused by the GW. Therefore, we can simply identify $|L|$ as the proper separation.

We now evaluate the geodesic deviation equation (6.4) in the local proper reference frame coordinates. From the conditions (6.5) it follows that we can replace the covariant time derivative operator $u^{\mu} \nabla_{\mu}$ with $\partial /(\partial t)$. Using $\vec{u}=\partial_{t}$ and $L^{\mu}=\left(0, L^{i}\right)$, we get

$$
\frac{d^{2} L^{i}(t)}{d t^{2}}=-R_{i 0 j 0}(t, 0) L^{j}(t) .
$$

Note that the key quantity entering into the equation, $R_{i 0 j 0}$, is gauge invariant in linearized theory, so we can use any convenient coordinate system to evaluate it. Using the expression (V A) for the Riemann tensor in terms of the TT gauge metric perturbation $h_{i j}^{\mathrm{TT}}$ we find

$$
\frac{d^{2} L^{i}}{d t^{2}}=\frac{1}{2} \frac{d^{2} h_{i j}^{\mathrm{T} T}}{d t^{2}} L^{j} .
$$

Integrating this equation using $L^{i}(t)=L_{0}^{i}+\delta L^{i}(t)$ with $|\delta L| \ll\left|L_{0}\right|$ gives

$$
\delta L^{i}(t)=\frac{1}{2} h_{i j}^{\mathrm{TT}}(t) L_{0}^{j}
$$


This equation is ideal to analyze an interferometric $\mathrm{GW}$ detector. We choose Cartesian coordinates such that the interferometer's two arms lie along the $x$ and $y$ axes, with the beam splitter at the origin. For concreteness, let us imagine that the GW propagates down the $z$-axis. Then, as discussed in Sec. V A, the only non-zero components of the metric perturbation are $h_{x x}^{\mathrm{TT}}=-h_{y y}^{\mathrm{TT}}=h_{+}$and $h_{x y}^{\mathrm{TT}}=h_{y x}^{\mathrm{TT}}=h_{\times}$, where $h_{+}(t-z)$ and $h_{\times}(t-z)$ are the two polarization components. We take the ends of one of the interferometer's two arms as defining the two nearby geodesics; the first geodesic is defined by the beam splitter at $x=0$, the second by the end-mirror. From Eq. (6.8), we then find that the distances $L=|L|$ of the arms end from the beam splitter vary with time as

$$
\begin{gathered}
\frac{\delta L_{x}}{L}=\frac{1}{2} h_{+}, \\
\frac{\delta L_{y}}{L}=-\frac{1}{2} h_{+} .
\end{gathered}
$$

(Here the subscripts $x$ and $y$ denote the two different arms, not the components of a vector). These distance variations are then measured via laser interferometry. Notice that the GW (which is typically a sinusoidally varying function) acts tidally, squeezing along one axis and stretching along the other. In this configuration, the detector is sensitive only to the + polarization of the GW. The $\times$ polarization acts similarly, except that it squeezes and stretches along a set of axes that are rotated with respect to the $x$ and $y$ axes by $45^{\circ}$. The force lines corresponding to the two different polarizations are illustrated in Fig. (10).

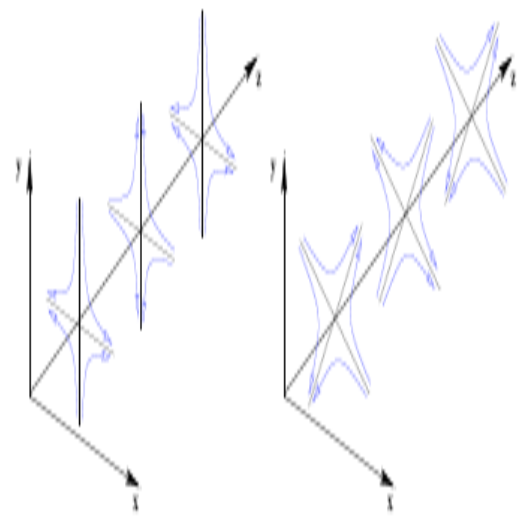

Fig (10). Lines of force associated to the + (left panel) and $\times$ (right panel) polarizations.

Of course, we don't expect nature to provide GWs that so perfectly align with our detectors. In general, we will need to account for the detector's antenna pattern, meaning that we will be sensitive to some weighted combination of the two polarizations, with the weights depending upon the location of a source on the sky, and the relative orientation of the source, the frequency and the detector [44, 88-90].

Finally, in our analysis so far of detection we have assumed that the only contribution to the metric perturbation is the GW contribution. However, in reality time-varying near zone gravitational fields produced by sources in the vicinity of the detector will also be present. From Eq. (6.7) we see that the quantity that is actually measured by interferometric detectors is the space-time-space-time or electric-type piece $R_{i 0 j 0}$ of the Riemann tensor (or more precisely the time-varying piece of this within the frequency band of the detector). From the general expression of Riemann tensor (see [38]), we see that $R_{i 0 j 0}$ contains contributions from both $h_{i j}^{\mathrm{TT}}$ describing GWs, and also additional terms describing the time-varying near zone gravitational fields. There is no way for the detector to separate these two contributions, and the time-varying near zone gravitational fields produced by motions of bedrock, air, human bodies, and tumbleweeds can all contribute to the output of the detector and act as sources of noise [92-94].

\section{A. The Generation of Gravitational Waves}

GWs are generated by the matter source term on the right hand side of the linearized Einstein equation

$$
\square \bar{h}_{\mu v}=-16 \pi T_{\mu v},
$$

cf. Eq. (5.6) (presented here in Lorentz gauge). In this section we will compute the leading order contribution to the spatial components of the metric perturbation for a source whose internal motions are slow compared to the speed of light ("slow-motion sources"). We will then compute the TT piece of the metric perturbation to obtain the standard quadrupole formula for the emitted radiation.

Eq. (6.9) can be solved by using a Green's function. A wave equation with source generically takes the form

$$
\square f(t, x)=s(t, x),
$$

where $f(t, x)$ is the radiative field, depending on time $t$ and position $x$, and $s(t, x)$ is a source function. Green's function $G\left(t, x ; t^{\prime}, x^{\prime}\right)$ is the field which arises due to a delta function source; it tells how much field is generated at the "field point" $(t, x)$ per unit source at the "source point" $\left(t^{\prime}, x^{\prime}\right)$ :

$$
\square G\left(t, x ; t^{\prime}, x^{\prime}\right)=\delta\left(t-t^{\prime}\right) \delta\left(x-x^{\prime}\right) .
$$

The field which arises from our actual source is then given by integrating Green's function against $s(t, x)$ :

$$
f(t, x)=\int d t^{\prime} d^{3} x^{\prime} G\left(t, x ; t^{\prime}, x^{\prime}\right) s\left(t^{\prime}, x^{\prime}\right) .
$$

The Green's function associated with the wave operator $\square$ is very well known (see, e.g., [95]):

$$
G\left(t, x ; t^{\prime}, x^{\prime}\right)=-\frac{\delta\left(t^{\prime}-\left[t-\left|x-x^{\prime}\right| / c\right]\right)}{4 \pi\left|x-x^{\prime}\right|} .
$$

The quantity $t-\left|x-x^{\prime}\right| / c$ is the retarded time; it takes into account the lag associated with the propagation of information from events at $x$ to position $x^{\prime}$. The speed of light $c$ has been restored here to emphasize the causal nature of this Green's function; we set it back to unity in what follows. 
Applying this result to Eq. (6.9), we find

$$
\bar{h}_{a b}(t, x)=4 \int d^{3} x^{\prime} \frac{T_{\mu \nu}\left(t-\left|x-x^{\prime}\right|, x^{\prime}\right)}{\left|x-x^{\prime}\right|} .
$$

Projected transverse and traceless, as already mentioned, the radiative degrees of freedom are contained entirely in the spatial part of the metric. First, consider the spatial part of the metric:

$$
\bar{h}_{i j}(t, x)=4 \int d^{3} x^{\prime} \frac{T^{i j}\left(t-\left|x-x^{\prime}\right|, x^{\prime}\right)}{\left|x-x^{\prime}\right|} .
$$

We have raised indices on the right-hand side, using the rule that the position of spatial indices in linearized theory is irrelevant.

We now evaluate this quantity at large distances from the source. This allows us to replace the factor $\left|x-x^{\prime}\right|$ in the denominator with $r=|x|$. The corresponding fractional errors scale as $\sim L / r$, where $L$ is the size of the source; these errors can be neglected. We also make the same replacement in the time argument of $T_{i j}$ :

$$
T_{i j}\left(t-\left|x-x^{\prime}\right|, x^{\prime}\right) \approx T_{i j}\left(t-r, x^{\prime}\right)
$$

Using the formula $\left|x-x^{\prime}\right|=r-n^{i} x^{\prime i}+O(1 / r)$ where $n^{i}=x^{i} / r$, we see that the fractional errors generated by the replacement (VI A) scale as $L / \tau$, where $\tau$ is the timescale over which the system is changing. This quantity is just the velocity of internal motions of the source (in units with $c=1$ ), and is therefore small compared to one by our assumption. These replacements give

$$
\bar{h}_{i j}(t, x)=\frac{4}{r} \int d^{3} x^{\prime} T^{i j}\left(t-r, x^{\prime}\right),
$$

which is the first term in a multipolar expansion of the radiation field.

Eq. (6.10) almost gives us the quadrupole formula that describes GW emission (at leading order). To get the remaining part there, we need to manipulate this equation a bit. The stress-energy tensor must be conserved, which means $\partial_{\mu} T^{\mu v}=0$ in linearized theory. Breaking this up into time and space components, we have the conditions

$$
\begin{aligned}
& \partial_{t} T^{00}+\partial_{i} T^{0 i}=0, \\
& \partial_{t} T^{0 i}+\partial_{j} T^{i j}=0 .
\end{aligned}
$$

From this, it follows that

$$
\partial_{t}^{2} T^{00}=\partial_{k} \partial_{l} T^{k l} .
$$

Multiplying both sides of this equation by $x^{i} x^{j}$, we first manipulate the left-hand side:

$$
\partial_{t}^{2} T^{00} x^{i} x^{j}=\partial_{t}^{2}\left(T^{00} x^{i} x^{j}\right) .
$$

Next, manipulate the right-hand side of Eq. (6.11); multiplying by $x^{i} x^{j}$, we obtain:

$$
\partial_{k} \partial_{l} T^{k l} x^{i} x^{j}=\partial_{k} \partial_{l}\left(T^{k l} x^{i} x^{j}\right)-2 \partial_{k}\left(T^{i k} x^{j}+T^{k j} x^{i}\right)+2 T^{i j} .
$$

This identity is easily verified by expanding the derivatives and applying the identity $\partial_{i} x^{j}=\delta_{i}{ }^{j}$. We thus have

$$
\partial_{t}^{2}\left(T^{00} x^{i} x^{j}\right)=\partial_{k} \partial_{l}\left(T^{k l} x^{i} x^{j}\right)-2 \partial_{k}\left(T^{i k} x^{j}+T^{k j} x^{i}\right)+2 T^{i j} .
$$

This yields

$$
\begin{aligned}
& \frac{4}{r} \int d^{3} x^{\prime} T_{i j}=\frac{4}{r} \int d^{3} x^{\prime}\left[\frac{1}{2} \partial_{t}^{2}\left(T^{00} x^{i} x^{j}\right)+\right. \\
& \left.+\partial_{k}\left(T^{i k} x^{j}+T^{k j} x^{i}\right)-\frac{1}{2} \partial_{k} \partial_{l}\left(T^{k l} x^{i i} x^{j}\right)\right] \\
& =\frac{2}{r} \int d^{3} x^{\prime} \partial_{t}^{2}\left(T^{00} x^{i} x^{j}\right) \\
& =\frac{2}{r} \frac{\partial^{2}}{\partial t^{2}} \int d^{3} x^{\prime} T^{00} x^{i i} x^{j} \\
& =\frac{2}{r} \frac{\partial^{2}}{\partial t^{2}} \int d^{3} x^{\prime} \rho x^{i} x^{j} .
\end{aligned}
$$

In going from the first to the second line, we used the fact that the second and third terms under the integral are divergences. Using Gauss's theorem, they can thus be recast as surface integrals; taking the surface outside the source, their contribution is zero. In going from the second to the third line, we used the fact that the integration domain is not time dependent, so we can take the derivatives out of the integral. Finally, we used the fact that $T^{00}$ is the mass density $\rho$. Defining the second moment $Q_{i j}$ of the mass distribution via

$$
Q_{i j}(t)=\int d^{3} x^{\prime} \rho\left(t, x^{\prime}\right) x^{i} x^{j},
$$

and combining Eqs. (6.10) and (6.12) we get

$$
\bar{h}_{i j}(t, x)=\frac{2}{r} \frac{d^{2} Q_{i j}(t-r)}{d t^{2}} .
$$

When we subtract the trace from $Q_{i j}$, we obtain the quadrupole momentum tensor:

$$
Q_{i j}=Q_{i j}-\frac{1}{3} \delta_{i j} Q, \quad Q=Q_{i i} .
$$

To complete the derivation, we must project out the nonTT pieces of the right-hand side of Eq. (6.13). Since we are working to leading order in $1 / r$, at each field point $x$, this operation reduces to algebraically projecting the tensor perpendicularly to the local direction of propagation $n=x / r$, and subtracting off the trace. It is useful to introduce the projection tensor,

$$
P_{i j}=\delta_{i j}-n_{i} n_{j} .
$$

This tensor eliminates vector components parallel to $n$, leaving only transverse components. Thus, 


$$
\bar{h}_{i j}^{T}=\bar{h}_{k l} P_{i k} P_{j l}
$$

is a transverse tensor. Finally, we remove the trace; what remains is

$$
h_{i j}^{\mathrm{TT}}=\bar{h}_{k l} P_{i k} P_{j l}-\frac{1}{2} P_{i j} P_{k l} \bar{h}_{k l} .
$$

Substituting Eq. (6.13) into (6.14), we obtain our final quadrupole formula:

$$
h_{i j}^{\mathrm{TT}}(t, x)=\frac{2}{r} \frac{d^{2} Q_{k l}(t-r)}{d t^{2}}\left[P_{i k}(n) P_{j l}(n)-\frac{1}{2} P_{k l}(n) P_{i j}(n)\right],
$$

or

$$
h_{i j}^{\mathrm{TT}}(t, x)=\frac{2 G}{r c^{4}} Q_{k l}(t-r) P_{i j k l} .
$$

One can now search for wave solutions of (6.9) from a system of masses undergoing arbitrary motions, and then obtain the power radiated. The result, assuming the source dimensions very small with respect to the wavelengths, (quadrupole approximation [26]), is that the power $\frac{d E}{d \Omega}$ radiated in a solid angle $\Omega$ is

$$
\frac{d E}{d \Omega}=\frac{G}{8 \pi c^{5}}\left(\frac{d^{3} Q_{i j}}{d t^{3}}\right)^{2}
$$

If one sums (6.16) over the two allowed polarizations, one obtains

$$
\begin{aligned}
& \sum_{p o l} \frac{d E}{d \Omega}=\frac{G}{8 \pi c^{5}}\left[\frac{d^{3} Q_{i j}}{d t^{3}} \frac{d^{3} Q_{i j}}{d t^{3}}-2 n_{i} \frac{d^{3} Q_{i j}}{d t^{3}} n_{k} \frac{d^{3} Q_{k j}}{d t^{3}}+\right. \\
& \left.-\frac{1}{2}\left(\frac{d^{3} Q_{i i}}{d t^{3}}\right)^{2}+\frac{1}{2}\left(n_{i} n_{j} \frac{d^{3} Q_{i j}}{d t^{3}}\right)^{2}+\frac{d^{3} Q_{i i}}{d t^{3}} n_{j} n_{k} \frac{d^{3} Q_{j k}}{d t^{3}}\right]
\end{aligned}
$$

where $\hat{n}$ is the unit vector in the radiation direction. The total radiation rate is obtained by integrating (6.17) over all directions of emission; the result is

$$
F^{G W}=\frac{d E}{d t}=-\frac{G\left\langle Q_{i j}^{(3)} Q^{(3) i j}\right\rangle}{45 c^{5}}
$$

where the index (3) represents the number of differentiations with respect to time, the symbol $<$ indicates that the quantity is averaged over several wavelengths.

\section{B. Extension to Sources with Non-Negligible Self Gravity}

Concerning our derivation of the quadrupole formula (6.15) we assumed the validity of the linearized Einstein equations. In particular, the derivation is not applicable to systems with weak (Newtonian) gravity whose dynamics are dominated by self-gravity, such as binary star systems ${ }^{8}$. This

${ }^{8}$ Stress energy conservation in linearized gravity, $\partial^{\mu} T_{\mu v}=0$, forces all bodies to move on geodesics of the Minkowski metric. shortcoming of the above linearized-gravity derivation of the quadrupole formula was first pointed out by Eddington. However, it is very straightforward to extend the derivation to encompass systems with non-negligible self gravity.

In full GR, we define the quantity $\bar{h}^{\mu v}$ via

$$
\sqrt{-g} g^{\mu v}=\eta^{\mu v}-\bar{h}^{\mu v}
$$

where $\eta^{\mu \nu} \equiv \operatorname{diag}(-1,1,1,1)$. When gravity is weak, this definition coincides with our previous definition of $\bar{h}^{\mu v}$ as a trace-reversed metric perturbation. We impose the harmonic gauge condition

$$
\partial_{\mu}\left(\sqrt{-g} g^{\mu v}\right)=\partial_{\mu} \bar{h}^{\mu v}=0 .
$$

In this gauge, the Einstein equation can be written as

$$
\square_{\mathrm{fl} l a t} \bar{h}^{\mu v}=-16 \pi\left(T^{\mu v}+t^{\mu v}\right)
$$

where $\square_{\text {flat }} \equiv \eta^{\mu v} \partial_{\mu} \partial_{v}$ is the flat-spacetime wave operator, and $t^{\mu \nu}$ is a pseudotensor that is constructed from $\bar{h}^{\mu v}$. Taking a coordinate divergence of this equation and using the gauge condition (6.19), stress-energy conservation can be written

$$
\partial_{\mu}\left(T^{\mu v}+t^{\mu v}\right)=0
$$

Eqs. (6.19)- (6.20) and (6.21) are precisely the same equations as are used in the linearized-gravity derivation of the quadrupole formula, except for the fact that the stress energy tensor $T^{\mu v}$ is replaced by $T^{\mu v}+t^{\mu v}$. Therefore the derivation of the last subsection carries over, with the modification that the formula (6.12) for $Q_{i j}$ is replaced by

$$
Q_{i j}(t)=\int d^{3} x^{\prime}\left[T^{00}\left(t, x^{\prime}\right)+t^{00}\left(t, x^{\prime}\right)\right] x^{i} x^{j} .
$$

In this equation the term $t^{00}$ describes gravitational binding energy, roughly speaking. For systems with weak gravity, this term is negligible in comparison with the term $T^{00}$ describing the rest-masses of the bodies. Therefore the quadrupole formula (6.15) and the original definition (6.12) of $Q_{i j}$ continue to apply to the more general situation considered here.

\section{Dimensional Analysis}

The rough form of the leading GW field that we just derived, Eq. (6.15), can be deduced using simple physical arguments. First, we define some moments of the mass distribution. The zeroth moment is just the mass itself:

$$
M_{0} \equiv \int \rho d^{3} x=M
$$

More accurately, this is the total mass-energy of the source. Next, we define the dipole moment:

$$
M_{1} \equiv \int \rho x_{i} d^{3} x=M L_{i} .
$$

$L_{i}$ is a vector with the dimension of length; it describes the displacement of the center of mass from the origin we chose. As such, $M_{1}$ is clearly not a very meaningful quantity - we can change its value simply by choosing a different origin. 
If our mass distribution exhibits internal motion, then moment of the mass current, $j_{i}=\rho v_{i}$, are also important. The first momentum is the spin angular momentum:

$$
S_{1} \equiv \int \rho v_{j} x_{k} \varepsilon_{i j k} d^{3} x=S_{i} .
$$

Finally, we look at the second momentum of the mass distribution:

$$
M_{2} \equiv \int \rho x_{i} x_{j} d^{3} x=M L_{i j}
$$

where $L_{i j}$ is a tensor with the dimension length squared.

Using dimensional analysis and simple physical arguments, it is simple to see that the first moment that can contribute to $\mathrm{GW}$ emission is $M_{2}$. Consider first $M_{0}$. We want to combine $M_{0}$ with the distance to our source, $r$, in such a way as to produce a dimensionless wavestrain $h$. The only way to do this (bearing in mind that the strain should fall off as $1 / r$, and restoring factors of $G$ and $c$ ) is to put

$$
h \sim \frac{G}{c^{2}} \frac{M_{0}}{r} .
$$

Conservation of mass-energy tells us that $M_{0}$ for an isolated source cannot vary dynamically. This $h$ cannot be radiative; it corresponds to a Newtonian potential, rather than a GW.

Let us consider now the momentum $M_{1}$. In order to get the right dimensions, we must take one time derivative:

$$
h \sim \frac{G}{c^{3}} \frac{d}{d t} \frac{M_{1}}{r} .
$$

The extra factor of $c$ converts the dimension of the time derivative to space, so that the whole expression is dimensionless. Think carefully about the derivative of $M_{1}$ :

$$
\frac{d M_{1}}{d t}=\frac{d}{d t} \int \rho x_{i} d^{3} x=\int \rho v_{i} d^{3} x=P_{i} .
$$

This is the total momentum of our source. Our guess for the form of a wave corresponding to $M_{1}$ becomes

$$
h \sim \frac{G}{c^{3}} \frac{P}{r} .
$$

Also this formula cannot describe a GW. The momentum of an isolated source must be conserved. By boosting into a different Lorentz frame, we can always set $P=0$. Terms like this can only be gauge artifacts; they do not correspond to radiation. Indeed, terms like (6.22) appear in the metric of a moving $\mathrm{BH}$, and correspond to the relative velocity of the $\mathrm{BH}$ and the observer [96].

Dimensional analysis tells us that radiation from $S_{1}$ must take the form

$$
h \sim \frac{G}{c^{4}} \frac{d}{d t} \frac{S_{1}}{r} .
$$

Conservation of angular momentum tells us that the total spin of an isolated system cannot change, so we must reject also this term. Finally, we examine $M_{2}$ :

$$
h \sim \frac{G}{c^{4}} \frac{d^{2}}{d t^{2}} \frac{M_{2}}{r} .
$$

There is no conservation principle that allows us to reject this term. This is the quadrupole formula we derived earlier, up to numerical factors.

In "normal" units, the prefactor of this formula turns out to be $G / c^{4}-$ a small number divided by a very big number. In order to generate interesting amounts of GWs, the variation quadrupole momentum must be enormous. The only interesting sources of GWs will be those which have very large masses undergoing extremely rapid variation; even in this case, the strain we expect from typical sources is tiny. The smallness of GWs reflects the fact that gravity is the weakest of the fundamental interactions.

\section{Numerical Estimates}

Consider a binary star system, with stars of mass $m_{1}$ and $m_{2}$ in a circular orbit with separation $R$. The quadrupole moment is given by

$$
Q_{i j}=\mu\left(x_{i} x_{j}-\frac{1}{3} R^{2} \delta_{i j}\right),
$$

where $x$ is the relative displacement, with $|x|=R$. We use the center-of-mass reference frame, and choose the coordinate axes so that the binary lies in the $x y$ plane, so $x=x_{1}=R \cos \Omega t, \quad y=x_{2}=R \sin \Omega t, \quad z=x_{3}=0$. Let us further choose to evaluate the field on the $z$ axis, so that $n$ points in the $z$-direction. The projection operators in Eq. (6.15) then simply serve to remove the $z j$ components of the tensor. Bearing this in mind, the quadrupole formula (6.15) yields

$$
h_{i j}^{\mathrm{TT}}=\frac{2 Q_{i j}}{r} .
$$

The quadrupole moment tensor is

$$
Q_{i j}=\mu R^{2}\left[\begin{array}{ccc}
\cos ^{2} \Omega t-\frac{1}{3} & \cos \Omega t \sin \Omega t & 0 \\
\cos \Omega t \sin \Omega t & \cos ^{2} \Omega t-\frac{1}{3} & 0 \\
0 & 0 & -\frac{1}{3}
\end{array}\right] \text {; }
$$

its second derivative is

$$
Q_{i j}=-2 \Omega^{2} \mu R^{2}\left[\begin{array}{ccc}
\cos 2 \Omega t & \sin 2 \Omega t & 0 \\
-\sin 2 \Omega t & -\cos 2 \Omega t & 0 \\
0 & 0 & 0
\end{array}\right] \text {. }
$$

The magnitude $h$ of a typical non-zero component of $h_{i j}^{\mathrm{TT}}$ is 


$$
h=\frac{4 \mu \Omega^{2} R^{2}}{r}=\frac{4 \mu M^{2 / 3} \Omega^{2 / 3}}{r} .
$$

We used Kepler's 3rd law ${ }^{9}$ to replace $R$ with powers of the orbital frequency $\Omega$ and the total mass $M=m_{1}+m_{2}$. The combination of masses here, $\mu M^{2 / 3}$, appears quite often in studies of $\mathrm{GW}$ emission from binaries; it motivates the definition of the chirp mass:

$$
M=\mu^{3 / 5} M^{2 / 5} .
$$

For the purpose of numerical estimate, we will take the members of the binary to have equal masses, so that $\mu=M / 4$ :

$$
h=\frac{M^{5 / 3} \Omega^{2 / 3}}{r} .
$$

Finally, we insert numbers corresponding to plausible sources:

$$
\begin{aligned}
& h \simeq 10^{-21}\left(\frac{M}{2 M_{\bullet}}\right)^{5 / 3}\left(\frac{1 \text { hour }}{P}\right)^{2 / 3}\left(\frac{1 \text { kiloparsec }}{r}\right) \\
& \simeq 10^{-22}\left(\frac{M}{2.8 M_{\star}}\right)^{5 / 3}\left(\frac{0.01 \mathrm{sec}}{P}\right)^{2 / 3}\left(\frac{100 \text { Megaparsecs }}{r}\right) .
\end{aligned}
$$

The first line corresponds roughly to the mass, distance and orbital period ( $P=2 \pi / \Omega$ ) expected for the many close binary white dwarf systems in our G alaxy. Such binaries are so common that they are likely to be a confusion limited source of GWs for space-based detectors, acting in some cases as an effective source of noise. The second line contains typical parameter values for binary neutron stars that are on the verge of spiralling together and merging. Such waves are targets for the ground-based detectors that have recently begun operations. The tiny magnitude of these waves illustrates why detecting GWs is so difficult. The emission of GWs costs energy and to compensate for the loss of energy, the radial separation $R$ between the two bodies must decrease. We shall now derive how the orbital frequency and GW frequency change in time, using Newtonian dynamics and the balance equation

$$
\frac{d E_{\text {orbit }}}{d t}=-P
$$

At Newtonian order, $E_{\text {orbit }}=-m_{1} m_{2} /(2 R)$. Thus, $\dot{R}=-2 / 3(R \Omega)\left(\dot{\Omega} / \Omega^{2}\right)$. As long as $\dot{\Omega} / \Omega^{2} \ll 1$, the radial velocity is smaller than the tangential velocity and the binary's motion is well approximated by an adiabatic sequence of quasi-circular orbits. Eq. (6.25) implies that the orbital frequency varies as

$$
\frac{\dot{\Omega}}{\Omega^{2}}=\frac{96}{5} v\left(\frac{G M \Omega}{c^{3}}\right)^{5 / 3},
$$

and the GW frequency $f_{\mathrm{GW}}=2 \omega$,

${ }^{9}$ In units with $G=1$, and for circular orbits of radius $R, R^{3} \Omega^{2}=M$

$$
\dot{f}_{\mathrm{GW}}=\frac{96}{5} \pi^{8 / 3}\left(\frac{M}{c^{3}}\right)^{5 / 3} f_{\mathrm{GW}}^{11 / 3} .
$$

Introducing the time to coalescence $\tau=t_{\text {coal }}-t$, and integrating Eq. (6.28), we get

$$
f_{\mathrm{GW}} \simeq 130\left(\frac{1.21 M_{\bullet}}{M}\right)^{5 / 8}\left(\frac{1 \mathrm{sec}}{\tau}\right)^{3 / 8} \mathrm{H} z,
$$

where $1.21 M_{\odot}$ is the chirp mass of a NS-NS binary. Eq. (6.28) predicts, e.g. coalescence times of $\sim 17 \mathrm{~min}, 2 \mathrm{sec}, 1 \mathrm{msec}$, for $f_{\mathrm{G} W} \sim 10,100,10^{3} \mathrm{~Hz}$. Using the above equations, it is straightforward to compute the relation between the radial separation and the GW frequency. We find

$$
R \simeq 300\left(\frac{M}{2.8 M_{\odot}}\right)^{1 / 3}\left(\frac{100 \mathrm{H} z}{f_{\mathrm{G} W}}\right)^{2 / 3} \mathrm{k} m .
$$

Finally, a useful quantity is the number of GW cycles, defined by

$$
N_{\mathrm{G} W}=\frac{1}{\pi} \int_{t_{\mathrm{i} i}}^{t_{\mathrm{t} i n}} \Omega(t) d t=\frac{1}{\pi} \int_{\Omega_{\mathrm{i} i}}^{\Omega_{\mathrm{fin}}} \frac{\Omega}{\dot{\Omega}} d \Omega .
$$

Assuming $\Omega_{\text {fin }} \ll \Omega_{\text {in }}$, we get

$$
N_{\mathrm{G} W} \simeq 10^{4}\left(\frac{M}{1.21 M_{\odot}}\right)^{-5 / 3}\left(\frac{f_{\mathrm{in}}}{10 \mathrm{H} z}\right)^{-5 / 3} .
$$

\section{GRAVITATIONAL WAVES FROM SOURCES IN NEWTONIAN MOTION}

We have now all the ingredient to characterize gravitational radiation with respect to the motion of the sources, i.e. with respect to different types of stellar encounters. Let us start with the Newtonian cases. With the above formalism, it is possible to estimate the amount of energy emitted in the form of GWs from a system of massive objects interacting among them $[34,35]$. Considering the quadrupole components for two bodies interacting in a Newtonian gravitational field, we have:

$$
\begin{aligned}
& Q_{x x}=\mu r^{2}\left(3 \cos ^{2} \varphi \sin ^{2} \theta-1\right), \\
& Q_{y y}=\mu r^{2}\left(3 \sin ^{2} \varphi \sin ^{2} \theta-1\right), \\
& Q_{z z}=\frac{1}{2} r^{2} \mu(3 \cos 2 \theta+1), \\
& Q_{x z}=Q_{z x}=r^{2} \mu\left(\frac{3}{2} \cos \varphi \sin 2 \theta\right), \\
& Q_{y z}=Q_{z y}=r^{2} \mu\left(\frac{3}{2} \sin 2 \theta \sin \varphi\right), \\
& Q_{x y}=Q_{y x}=r^{2} \mu\left(\frac{3}{2} \sin ^{2} \theta \sin 2 \varphi\right),
\end{aligned}
$$


where the masses $m_{1}$ and $m_{2}$ have polar coordinates $\left\{r_{i} \cos \theta \cos \varphi, r_{i} \cos \theta \sin \varphi, r_{i} \sin \theta\right\}$ with $i=1,2$. We will work in the equatorial plane $(\theta=\pi / 2)$. The origin of the motions is taken at the center of mass. Such components can be differentiated with respect to time, as in Eq. (6.18), in order to derive the amount of gravitational radiation in the various Newtonian orbital motions.

\section{A. Gravitational Wave Luminosity from Circular and Elliptical Orbits}

The first case we are going to consider is that of closed circular and elliptical orbits. Using Eq. (2.14), let us derive the angular velocity equation

$$
\phi=\frac{\sqrt{G l\left(m_{1}+m_{2}\right)}(\varepsilon \cos \phi+1)^{2}}{l^{2}}
$$

and then, from Eqs. (7.1), the third derivatives of quadrupolar components for the elliptical orbits are:

$$
\begin{aligned}
\frac{d^{3} Q_{x x}}{d t^{3}} & =\beta(24 \cos \phi+\varepsilon(9 \cos 2 \phi)+11)) \sin \phi \\
\frac{d^{3} Q_{y y}}{d t^{3}} & =-\beta(24 \cos \phi+\varepsilon(13+9 \cos 2 \phi)) \sin \phi) \\
\frac{d^{3} Q_{z z}}{d t^{3}} & =-2 \beta \varepsilon \sin \phi \\
\frac{d^{3} Q_{x y}}{d t^{3}} & =\beta(24 \cos \phi+\varepsilon(11+9 \cos 2 \phi)) \sin \phi)
\end{aligned}
$$

where

$$
\beta=\frac{\left.G l\left(m_{1}+m_{2}\right)\right)^{3 / 2} \mu(\varepsilon \cos \phi+1)^{2}}{l^{4}} .
$$

Being

$$
\begin{aligned}
& Q_{i j}^{(3)} Q^{(3) i j}=\frac{G^{3}}{l^{5}}\left[\left(m_{1}+m_{2}\right)^{3} \mu^{2}(1+\varepsilon \cos \phi)^{4}\right. \\
& \left(415 \varepsilon^{2}+3(8 \cos \phi+3 \varepsilon \cos 2 \phi)\right. \\
& \left.(72 \cos \phi+\varepsilon(70+27 \cos 2 \phi))) \sin \phi^{2}\right]
\end{aligned}
$$

the total power radiated is given by

$$
\frac{d E}{d t}=\frac{G^{3}}{45 c^{5} l^{5}} f(\phi),
$$

where

$$
\begin{aligned}
& f(\phi)=\left(m_{1}+m_{2}\right)^{3} \mu^{2}(1+\varepsilon \cos \phi)^{4} \times \\
& \left(415 \varepsilon^{2}+3(8 \cos \phi+3 \varepsilon \cos 2 \phi) \times\right. \\
& (72 \cos \phi+\varepsilon(70+27 \cos 2 \phi))) \sin \phi^{2} .
\end{aligned}
$$

The total energy emitted in the form of gravitational radiation, during the interaction, is :

$$
\Delta E=\int_{0}^{\infty}\left|\frac{d E}{d t}\right| d t .
$$

From Eq. (2.4), we can adopt the angle $\phi$ as a suitable integration variable. In this case, the energy emitted for $\phi_{1}<\phi<\phi_{2}$ is

$$
\Delta E\left(\phi_{1}, \phi_{2}\right)=\frac{G^{3}}{45 c^{5} l^{5}} \int_{\varphi_{1}}^{\varphi_{2}} f(\phi) d \phi,
$$

and the total energy can be determined from the previous relation in the limits $\varphi_{1} \rightarrow 0$ and $\varphi_{2} \rightarrow \pi$. Thus, one has

$$
\Delta E=\frac{G^{4} \pi\left(m_{1}+m_{2}\right)^{3} \mu^{2}}{l^{5} c^{5}} F(\varepsilon)
$$

where $F(\varepsilon)$ depends on the initial conditions only and it is given by

$$
F(\varepsilon)=\frac{\left(13824+102448 \varepsilon^{2}+59412 \varepsilon^{4}+2549 \varepsilon^{6}\right)}{2880} .
$$

In other words, the gravitational wave luminosity strictly depends on the configuration and kinematics of the binary system.

\section{B. Gravitational Wave Luminosity from Parabolic and Hyperbolic Orbits}

In this case, we use Eq. (2.15) and Eq. (7.1) to calculate the quadrupolar formula for parabolic and hyperbolic orbits. The angular velocity is

$$
\phi=l^{2} L(\varepsilon \cos \varphi+1)^{2},
$$

and the quadrupolar derivatives are

$$
\frac{d^{3} Q_{x x}}{d t^{3}}=\rho(24 \cos \phi+\varepsilon(9 \cos 2 \phi+11)) \sin \phi,
$$

$$
\begin{aligned}
\frac{d^{3} Q_{y y}}{d t^{3}} & =-\rho(24 \cos \phi+\varepsilon(13+9 \cos 2 \phi)) \sin \phi), \\
\frac{d^{3} Q_{z z}}{d t^{3}} & =-2 \rho \varepsilon \sin \phi, \\
\frac{d^{3} Q_{x y}}{d t^{3}} & =-\frac{3}{2} \rho(\varepsilon \cos \phi+1)^{2}(5 \varepsilon \cos \phi+8 \cos 2 \phi+3 \varepsilon \cos 3 \phi),
\end{aligned}
$$

where

$\rho=l^{4} L^{3} \mu(\varepsilon \cos \phi+1)^{2}$.

The radiated power is given by

$\frac{d E}{d t}=-\frac{G \rho^{2}}{120 c^{5}} \times$

$\left[314 \varepsilon^{2}+(1152 \cos (\phi+187 \varepsilon \cos 2 \phi\right.$

$-3(80 \cos 3 \phi+30 \varepsilon \cos 4 \phi+48 \cos 5 \phi+9 \varepsilon \cos 6 \phi)) \varepsilon$

$-192 \cos 4 \phi+576]$, 
then

$$
\frac{d E}{d t}=-\frac{G l^{8} L^{6} \mu^{2}}{120 c^{5}} f(\phi),
$$

where $f(\varphi)$, in this case, is

$$
\begin{aligned}
& f(\phi)=\left(314 \varepsilon^{2}+(1152 \cos (\phi+187 \varepsilon \cos 2 \phi-3(80 \cos 3 \phi\right. \\
& +30 \varepsilon \cos 4 \phi+48 \cos 5 \phi+9 \varepsilon \cos 6 \phi)) \varepsilon+\phi \\
& -192 \cos 4 \phi+576) .
\end{aligned}
$$

Then using Eq. (6.18), the total energy emitted in the form of gravitational radiation, during the interaction as a function of $\phi$, is given by

$$
\begin{aligned}
& \Delta E\left(\phi_{1}, \phi_{2}\right)=-\frac{1}{480 c^{5}} d \phi \times \\
& \left(G l^{8} L^{6} \pi\left(1271 \varepsilon^{6}+24276 \varepsilon^{4}+34768 \varepsilon^{2}+4608\right) \mu^{2}\right)
\end{aligned}
$$

and the total energy can be determined from the previous relation in the limits $\varphi_{1} \rightarrow-\pi$ and $\varphi_{2} \rightarrow \pi$ in the parabolic case. Thus, one has

$$
\Delta E=-\frac{\left(G l^{8} L^{6} \pi \mu^{2}\right.}{480 c^{5}} F(\varepsilon),
$$

where $F(\varepsilon)$ depends on the initial conditions only and it is given by

$$
F(\varepsilon)=\left(1271 \varepsilon^{6}+24276 \varepsilon^{4}+34768 \varepsilon^{2}+4608\right) .
$$

In the hyperbolic case, we have that the total energy is determined in the limits $\phi_{1} \rightarrow \frac{-3 \pi}{4}$ and $\phi_{2} \rightarrow \frac{-3 \pi}{4}$, i.e.

$$
\Delta E=-\frac{G l^{8} L^{6} \mu^{2}}{201600 c^{5}} F(\varepsilon),
$$

where $F(\varepsilon)$ depends on the initial conditions only and is given by

$$
\begin{aligned}
& F(\varepsilon)=\left[315 \pi\left(1271 \varepsilon^{6}+24276 \varepsilon^{4}+34768 \varepsilon^{2}+4608\right)+\right. \\
& +16 \varepsilon\left[\varepsilon \left[\varepsilon \left(926704 \sqrt{2}-7 \varepsilon\left(3319 \varepsilon^{2}-32632 \sqrt{2} \varepsilon\right.\right.\right.\right. \\
& +55200))-383460]+352128 \sqrt{2}]]
\end{aligned}
$$

As above, the gravitational wave luminosity strictly depends on the configuration and kinematics of the binary system.

\section{Gravitational Wave Amplitude from Elliptical Orbits}

Beside luminosity, we can characterize also the GW amplitude starting from the motion of sources. In the case of a binary system and a single amplitude component, it is straightforward to show that $h^{11}=-\frac{2 G}{R c^{4}} \frac{G\left(m_{1}+m_{2}\right) \mu(13 \varepsilon \cos \phi+12 \cos 2 \phi+\varepsilon(4 \varepsilon+3 \cos 3 \phi))}{2 l}$,

$h^{22}=\frac{2 G}{R c^{4}} \frac{G\left(m_{1}+m_{2}\right) \mu(17 \varepsilon \cos \phi+12 \cos 2 \phi+\varepsilon(8 \varepsilon+3 \cos 3 \phi))}{2 l}$,

$h^{12}=h^{21}=-\frac{2 G}{R c^{4}} \frac{G\left(m_{1}+m_{2}\right) \mu(13 \varepsilon \cos \phi+12 \cos 2 \phi+\varepsilon(4 \varepsilon+3 \cos 3 \phi))}{2 l}$, so that the expected strain amplitude $h \diamond\left(h_{11}^{2}+h_{22}^{2}+2 h_{12}^{2}\right)^{1 / 2}$ turns out to be

$$
\begin{aligned}
& h=\frac{G^{3}\left(m_{1}+m_{2}\right) \mu^{2}}{c^{4} R l^{2}} \times \\
& \left(3(13 \varepsilon \cos \phi+12 \cos 2 \phi+\varepsilon(4 \varepsilon+3 \cos 3 \phi))^{2}\right. \\
& \left.+(17 \varepsilon \cos \phi+12 \cos 2 \phi+\varepsilon(8 \varepsilon+3 \cos 3 \phi))^{2}\right)^{\frac{1}{2}},
\end{aligned}
$$

which, as before, strictly depends on the initial conditions of the stellar encounter. A remark is in order at this point. A monochromatic gravitational wave has, at most, two independent degrees of freedom. In fact, in the TT gauge, we have $h_{+}=h_{11}+h_{22}$ and $h_{x}=h_{12}+h_{21}$ (see e.g. [97]). As an example, the amplitude of gravitational wave is sketched in Fig. (11) for a stellar encounter, in Newtonian motion, close to the Galactic Center. The adopted initial parameters are typical of a close impact and are assumed to be $b=1$ AU for the impact factor and $v_{0}=200 \mathrm{Km} \mathrm{s}^{-1}$ for the initial velocity, respectively. Here, we have fixed $m_{1}=m_{2}=1.4 M_{\odot}$. The impact parameter is defined as $L=b v$ where $L$ is the angular momentum and $v$ the incoming velocity. We have chosen a typical velocity of a star in the galaxy and we are considering, essentially,

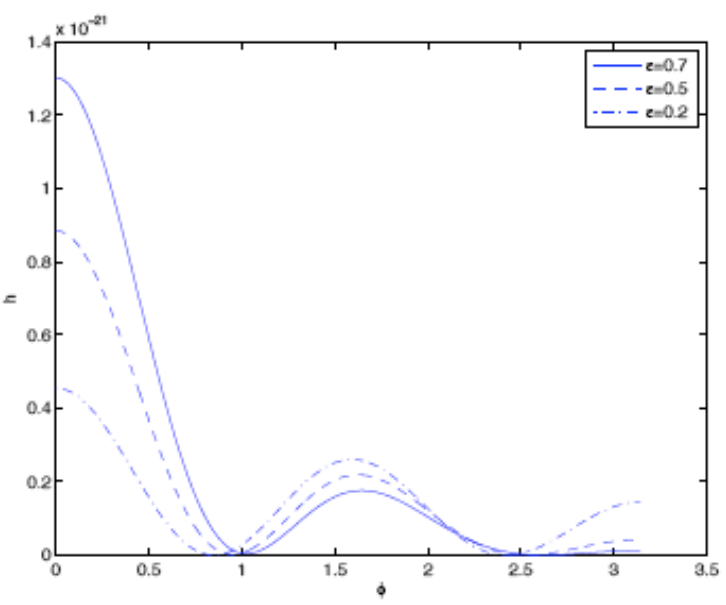

Fig. (11). The gravitational wave-forms from elliptical orbits shown as function of the polar angle $\phi$. We have fixed $m_{1}=m_{2}=1.4 M_{\odot} \cdot m_{2}$ is considered at rest while $m_{1}$ is moving with initial velocity $v_{0}=200 \mathrm{Km} \mathrm{s}^{-1}$ and an impact parameter $b=1$ AU. The distance of the GW source is assumed to be $R=8$ kpc and the eccentricity is $\varepsilon=0.2,0.5,0.7$. 
compact objects with masses comparable to the Chandrasekhar limit $\left(\sim 1.4 M_{\odot}\right)$. This choice is motivated by the fact that ground-based experiments like VIRGO or LIGO expect to detect typical GW emissions from the dynamics of these objects or from binary systems composed by them (see e.g. [43]).

\section{Gravitational Wave Amplitude from Parabolic and Hyperbolic Orbits}

The single components of amplitude for a parabolic and hyperbolic orbits are

$$
\begin{aligned}
& h^{11}=-\frac{G l^{2} L^{2} \mu}{R c^{4}}(13 \varepsilon \cos \phi+12 \cos 2 \phi+\varepsilon(4 \varepsilon+3 \cos 3 \phi)), \\
& h^{22}=\frac{G l^{2} L^{2} \mu}{R c^{4}}(17 \varepsilon \cos \phi+12 \cos 2 \phi+\varepsilon(8 \varepsilon+3 \cos 3 \phi)), \\
& h^{12}=h^{21}=-\frac{3 G l^{2} L^{2} \mu}{R c^{4}}(4 \cos \phi+\varepsilon(\cos 2 \phi+3)) \sin \phi,
\end{aligned}
$$

and then the expected strain amplitude is

$$
\begin{aligned}
& h=\frac{2 l^{4} L^{4} \mu^{2}}{c^{4} R} \times\left(10 \varepsilon^{4}+9 \varepsilon^{3} \cos 3 \phi+59 \varepsilon^{2} \cos 2 \phi\right. \\
& \left.+59 \varepsilon^{2}+\left(47 \varepsilon^{2}+108\right) \varepsilon \cos \phi+36\right)^{\frac{1}{2}},
\end{aligned}
$$

which, as before, strictly depends on the initial conditions of the stellar encounter. We note that the gravitational wave amplitude has the same analytical expression for both cases and differs only for the value of $\varepsilon$ which is $\varepsilon=1$ if the motion is parabolic and the polar angle range is $\phi \in(-\pi, \pi)$, while it is $\varepsilon>1$ and $\phi \in(-\pi, \pi)$ for hyperbolic orbits. In these cases, we have non-returning objects.

The amplitude of the gravitational wave is sketched in Figs. (12 and 13) for stellar encounters close to the Galactic Center. As above, we consider a close impact and assume $b=1 \mathrm{AU} \mathrm{cm}, v_{0}=200 \mathrm{Km} \mathrm{s}^{-1}$ and $m_{1}=m_{2}=1.4 M_{\odot}$. In summary, we can say that also in the case of Newtonian motion of sources, the orbital features characterize GW luminosities and amplitudes.

\section{GRAVITATIONAL WAVES FROM SOURCES IN RELATIVISTIC MOTION}

It is straighforward to extend the above considerations to orbital motions containing Post - Newtonian corrections. It is clear that GW luminosity and amplitude are strictly dependent on the parameter $\left(\frac{v}{c}\right)$ considered at various order of approximation and, as discussed above, the global feature of orbits fully characterize the gravitational emission. Now we study how the waveforms depend on the dynamics of binary and colliding systems and how relativistic corrections modulate the features of gravitational radiation.

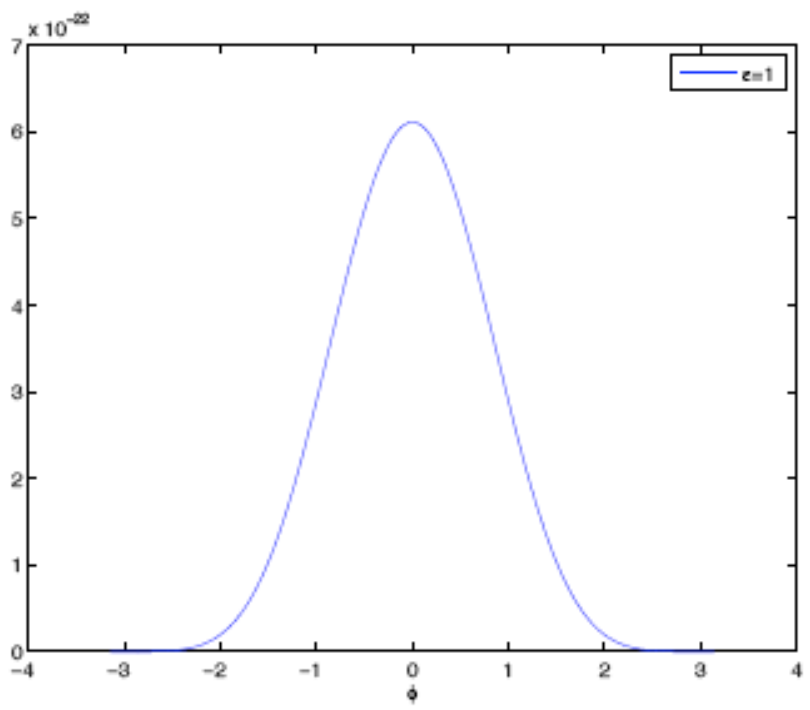

Fig. (12). The gravitational wave-forms for a parabolic encounter as a function of the polar angle $\phi$. As above, $m_{1}=m_{2}=1.4 M_{\odot}$ and $m_{2}$ is considered at rest. $m_{1}$ is moving with initial velocity $v_{0}=200 \mathrm{Km} \mathrm{s}^{-1}$ with an impact parameter $b=1 \mathrm{AU}$. The distance of the GW source is assumed at $R=8 \mathrm{kpc}$. The eccentricity is $\varepsilon=1$.

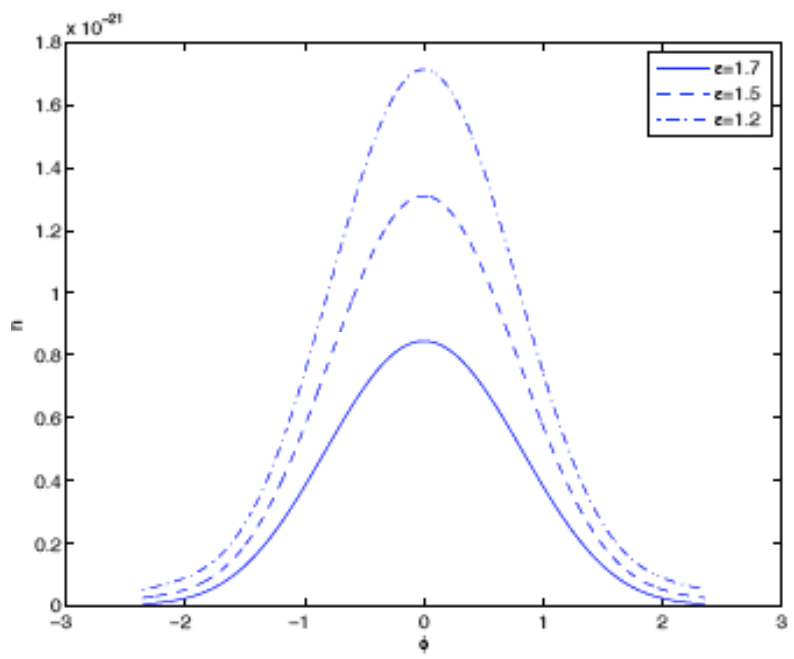

Fig. (13). The gravitational wave-forms for hyperbolic encounters as function of the polar angle $\phi$. As above, we have fixed $m_{1}=m_{2}=1.4 M_{\odot} \cdot m_{2}$ is considered at rest while $m_{1}$ is moving with initial velocity $v_{0}=200 \mathrm{Km} \mathrm{s}^{-1}$ and an impact parameter $b=1 \mathrm{AU}$. The distance of the source is assumed at $R=8 \mathrm{kpc}$. The eccentricity is assumed with the values $\varepsilon=1.2,1.5,1.7$.

\section{A. Inspiralling Waveform Including Post-Newtonian Corrections}

As we have shown in the above section the PN method involves an expansion around the Newtonian limit keeping terms of higher order in the small parameter $[8,98,99]$

$$
\varepsilon \sim \frac{v^{2}}{c^{2}} \sim\left|h_{\mu v}\right| \sim\left|\frac{\partial_{0} h}{\partial_{i} h}\right|^{2} \sim\left|\frac{T^{0 i}}{T^{00}}\right| \sim\left|\frac{T^{i j}}{T^{00}}\right| .
$$


In order to be able to determine the dynamics of binary systems with a precision acceptable for detection, it has been necessary to compute the force determining the motion of the two bodies and the amplitude of the gravitational radiation with a precision going beyond the quadrupole formula. For nonspinning BHs, the two-body equations of motion and the GW flux are currently known through 3.5PN order [100]. Specifically if we restrict the discussion to circular orbits, as Eq. (6.26) shows, there exists a natural adiabatic parameter $\dot{\Omega} / \Omega^{2} \cong O\left[(v / c)^{5}\right]$. Higher-order PN corrections to Eq. (6.26) have been computed [100, 101], yielding the general equation:

$$
\frac{\dot{\Omega}}{\Omega^{2}}=\frac{96}{5} v v_{\Omega}^{5 / 3} \sum_{k=0}^{7} \Omega_{(k / 2) \mathrm{PN}} v_{\Omega}^{k / 3}
$$

where $G=1=c$ and where we define $v_{\omega} \equiv(M \omega)^{1 / 3}$. The PN-order is given by $\Omega_{(k / 2) P N}$ which is, up to $k=7$,

$$
\begin{gathered}
\Omega_{0 \mathrm{PN}}=1, \\
\Omega_{0.5 \mathrm{PN}}=0, \\
\Omega_{1 \mathrm{PN}}=-\frac{743}{336}-\frac{11}{4} v, \\
\Omega_{1.5 \mathrm{PN}}=4 \pi+\left[-\frac{47}{3} \frac{S_{L}}{M^{2}}-\frac{25}{4} \frac{\delta m}{M} \frac{\Sigma_{L}}{M^{2}}\right], \\
\Omega_{2 \mathrm{PN}}=\frac{34103}{18144}+\frac{13661}{2016} v+\frac{59}{18} v^{2}- \\
\frac{1}{48} v \chi_{1} \chi_{2}\left[247\left(\hat{S}_{1} \cdot \hat{S}_{2}\right)-721\left(\hat{\mathbf{L}} \cdot \hat{S}_{1}\right)\left(\hat{\mathbf{L}} \cdot \hat{S}_{2}\right)\right], \\
\Omega_{2.5 \mathrm{PN}}=-\frac{1}{672}(4159+15876 v) \pi+\left[\left(-\frac{31811}{1008}+\right.\right. \\
\left.\left.\frac{5039}{84} v\right) \frac{S_{L}}{M^{2}}+\left(-\frac{473}{84}+\frac{1231}{56} v\right) \frac{\delta m}{M} \frac{\Sigma_{L}}{M^{2}}\right],
\end{gathered}
$$

$$
\begin{aligned}
& \Omega_{3 \mathrm{PN}}=\left(\frac{16447322263}{139708800}-\frac{1712}{105} \gamma_{E}+\frac{16}{3} \pi^{2}\right)+ \\
& \left(-\frac{56198689}{217728}+\frac{451}{48} \pi^{2}\right) v+ \\
& \frac{541}{896} v^{2}-\frac{5605}{2592} v^{3}-\frac{856}{105} \log \left[16 v^{2}\right], \\
& \Omega_{3.5 \mathrm{PN}}=\left(-\frac{4415}{4032}+\frac{358675}{6048} v+\frac{91495}{1512} v^{2}\right) \pi .
\end{aligned}
$$

We denote $\mathbf{L}=\mu X \times V$ the Newtonian angular momentum (with $X$ and $V$, as above, the two-body centerof-mass radial separation and relative velocity), and $\hat{\mathbf{L}}=\mathbf{L} /|\mathbf{L}| ; S_{1}=\chi_{1} m_{1}^{2} \hat{S}_{1}$ and $S_{2}=\chi_{2} m_{2}^{2} \hat{S}_{2}$ are the spins of the two bodies (with $\hat{S}_{1,2}$ unit vectors, and $0<\chi_{1,2}<1$ for BHs) and

$$
\mathbf{S} \equiv \mathbf{S}_{1}+\mathbf{S}_{2}, \quad \sum \equiv M\left[\frac{\mathbf{S}_{2}}{m_{2}}-\frac{\mathbf{S}_{1}}{m_{1}}\right] .
$$

Finally, $\delta m=m_{1}-m_{2}$ and $\gamma_{E}=0.577 \ldots$ is Euler's constant.

It is instructive to compute the relative contribution of the PN terms to the total number of GW cycles accumulating in the frequency band of LIGO/VIRGO. In Table II, we list the figures obtained by plugging Eq. (8.2) into Eq. (6.30) [8]. As final frequency, we use the innermost stable circular orbit (ISCO) of a point particle in Schwarzschild $\mathrm{BH}$ $\left[f_{\mathrm{G} W}^{\mathrm{ISCO}} \simeq 4400 /\left(M / M_{\odot}\right) \mathrm{Hz}\right]$.

\section{B. The Full Waveform: Inspiral, Merger and Ring-Down}

After the two BHs merge, the system settles down to a Kerr BH and emits quasi-normal modes (QNMs), [102, 103]. This phase is commonly known as the ring-down (RD) phase. Since the QNMs have complex frequencies totally determined by the BH's mass and spin, the RD waveform is a

Table 2. Post-Newtonian Contributions to the Number of GW Cycles Accumulated from $\Omega_{\text {in }}=\pi \times 10 \mathrm{~Hz}$ to $\Omega_{\text {fin }}=\Omega^{\mathrm{ISCO}}=1 /\left(6^{3 / 2} M\right)$ for Binaries Detectable by LIGO and VIRGO. We Denote $\kappa_{i}=\hat{S}_{i} \cdot \hat{\mathbf{L}}$ and $\xi=\hat{\mathbf{S}}_{1} \cdot \hat{\mathbf{S}}_{2}$

\begin{tabular}{|c|c|c|}
\hline & $(10+10) M \odot$ & $(1.4+1.4) M \odot$ \\
\hline Newtonian & 601 & 16034 \\
\hline $1 \mathrm{PN}$ & +59.3 & +441 \\
\hline $1.5 \mathrm{PN}$ & $-51.4+16.0 \kappa 1 \chi 1+16.0 \kappa 2 \chi 2$ & $-211+5.7 \kappa 1 \chi 1+65.7 \kappa 2 \chi 2$ \\
\hline $2 \mathrm{PN}$ & $+4.1-3.3 \kappa 1 \kappa 2 \chi 1 \chi 2+1.1 \xi \chi 1 \chi 2$ & $+9.9-8.0 \kappa 1 \kappa 2 \chi 1 \chi 2+2.8 \xi \chi 1 \chi 2$ \\
\hline $2.5 \mathrm{PN}$ & $-7.1+5.5 \kappa 1 \kappa 1+5.5 \kappa 2 \chi 2$ & $-11.7+9.0 \kappa 1 \chi_{1} 1+9.0 \kappa 2 \chi 2$ \\
\hline $3 \mathrm{PN}$ & +2.2 & +2.6 \\
\hline $3.5 \mathrm{PN}$ & -0.8 & -0.9 \\
\hline
\end{tabular}




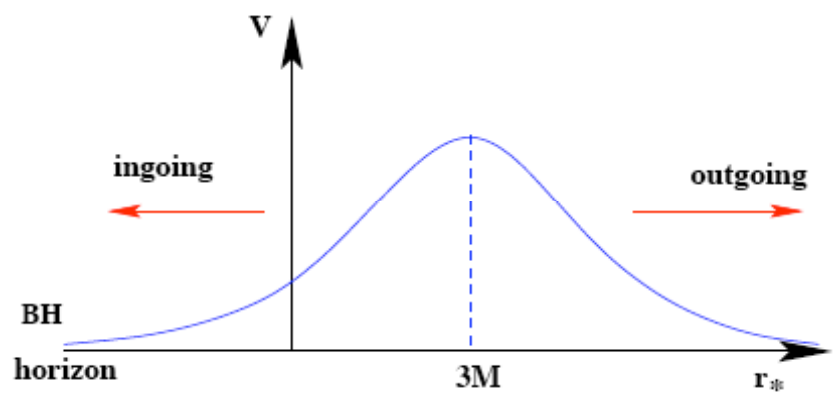

Fig. (14). We sketch the curvature potential as function of the tortoise coordinate $r^{*}$ associated to metric perturbations of a Schwarzschild BH.

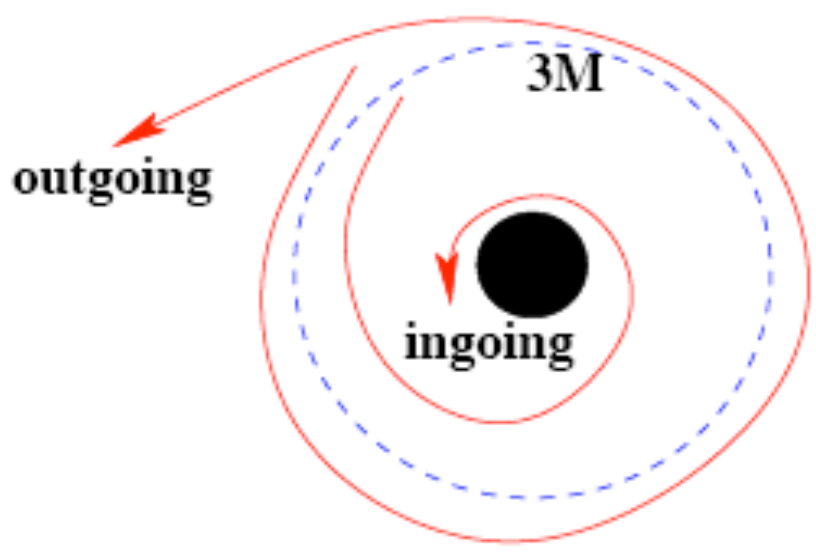

Fig (15). The potential peaks at the last unstable orbit for a massless particle (the light ring). Ingoing modes propagate toward the $\mathrm{BH}$ horizon, whereas outgoing modes propagate away from the source.

superposition of damped sinusoidals. The inspiral and RD waveforms can be computed analytically. What about the merger? Since the nonlinearities dominate, the merger would be described at best and utterly through numerical simulations of Einstein equations. However, before numerical relativity (NR) results became available, some analytic approaches were proposed. In the test-mass limit, $v \ll 1$, Refs. [103, 104] realized a long time ago that the basic physical reason underlying the presence of a universal merger signal was that when a test particle falls below $3 M$ (the unstable light storage ring of Schwarzschild), the GW that it generates is strongly filtered by the curvature potential barrier centered around it (see Fig. 14). For the equal-mass case $v=1 / 4$, Price and Pullin [105] proposed the so-called close-limit approximation, which consists in switching from the two-body description to the one-body description (perturbed-BH) close to the light-ring location. Based on these observations, the effective-one-body (EOB) resummation scheme [19] provided a first example of full waveform by (i) resumming the PN Hamiltonian, (ii) modeling the merger as a very short (instantaneous) phase and (iii) matching the end of the plunge (around the lightring) with the RD phase (see Ref. [106] where similar ideas were developed also in NR). The matching was initially done using only the least damped QNM whose mass and spin were determined by the binary BH energy and angular momentum at the end of the plunge. An example of full waveform is given in Fig. $(\mathbf{1 4}, \mathbf{1 7})$.

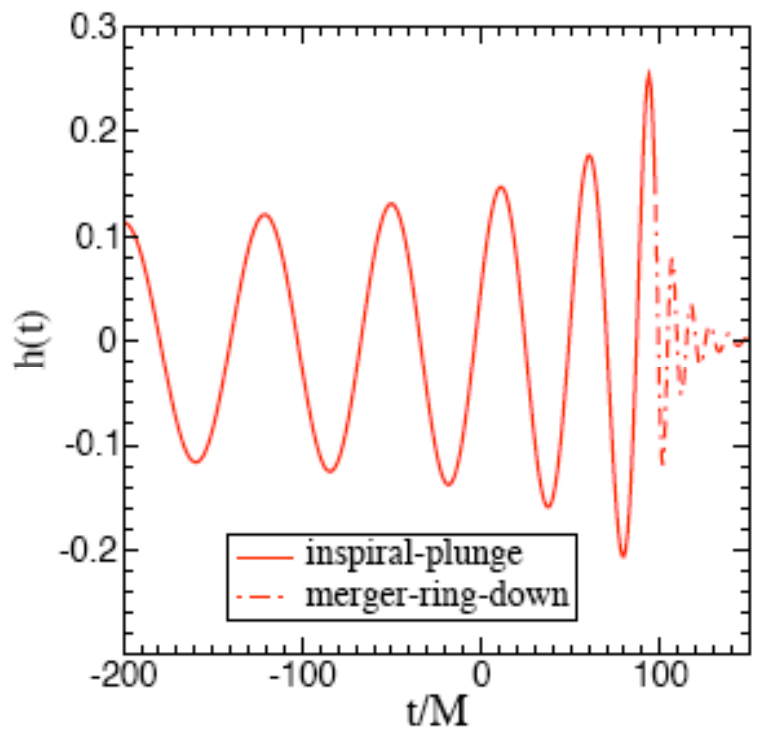

Fig (16). GW signal from an equal-mass nonspinning BH binary as predicted at 2.5PN order by Buonanno and Damour (2000) in Ref. [19]. The merger is assumed almost instantaneous and one QNM is included.

Today, with the results in NR, we are in the position of assessing the closeness of analytic to numerical waveforms for inspiral, merger and RD phases. In Fig. (16), we show some first-order comparisons between the EOB-analytic and NR waveforms [107] (see also Ref. [108]). Similar results for the inspiral phase but using PN theory [100, 101] (without resummation) at 3.5PN order are given in Refs. [107, 108]. So far, the agreement is qualitatively good, but more accurate simulations, starting with the $\mathrm{BHs}$ farther apart, are needed to draw robust conclusions.

Those comparisons are suggesting that it should be possible to design purely analytic templates with the full numerics used to guide the patching together of the inspiral and RD waveforms. This is an important avenue to template construction as eventually hundreds of thousands of waveform templates may be needed to extract the signal from the noise, an impossible demand for NR alone.

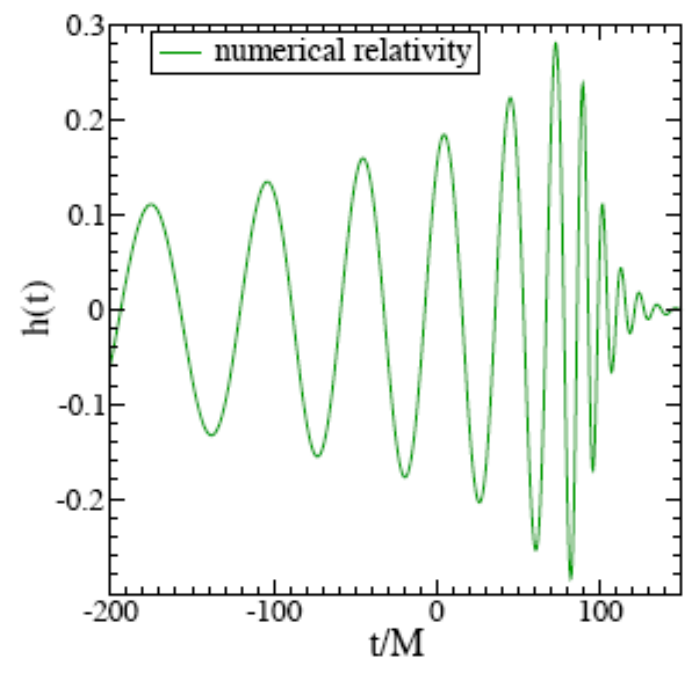

Fig (17). GW signal from an equal-mass BH binary with a small spin $\chi_{1}=\chi_{2}=0.06$ obtained in full GR by Pretorius [107]. 


\section{GRAVITATIONAL WAVES GRAVITOMAGNETIC CORRECTIONS}

WITH

In this section, we are going to study the evolution of compact binary systems, formed through the capture of a moving (stellar) mass $m$ by the gravitational field, whose source is a massive MBH of mass $M$ where $m \ll M$. One expects that small compact objects $\left(1 \div 20 M_{\odot}\right)$ from the surrounding stellar population will be captured by these black holes following many-body scattering interactions at a relatively high rate [109]. It is well known that the capture of stellar-mass compact objects by massive MBHs could constitute, potentially, a very important target for LISA [110, 111]. However, dynamics has to be carefully discussed in order to consider and select all effects coming from standard stellar mass objects inspiralling over MBHs.

In the first part of this review, we have shown that, in the relativistic weak field approximation, when considering higher order corrections to the equations of motion, gravitomagnetic effects in the theory of orbits, can be particularly significant, leading also to chaotic behaviors in the transient regime dividing stable from unstable trajectories. Generally, such contributions are discarded since they are considered too small. However, in a more accurate analysis, this is not true and gravitomagnetic corrections could give peculiar characterization of dynamics [65, 88-90].

According to these effects, orbits remain rather eccentric until the final plunge, and display both extreme relativistic perihelion precession and Lense-Thirring [69, 112, 113] precession of the orbital plane due to the spin of $\mathrm{MBH}$, as well as orbital decay. In [114], it is illustrated how the measured GW-waveforms can effectively map out the spacetime geometry close to the MBH. In [32, 33], the classical orbital motion (without relativistic corrections in the motion of the binary system) has been studied in the extreme mass ratio limit $m \ll M$, assuming the stellar system density and richness as fundamental parameters. The conclusions have been that

- the GW-waveforms have been characterized by the orbital motion (in particular, closed or open orbits give rise to very different $\mathrm{GW}$-production and waveform shapes);

- in rich and dense stellar clusters, a large production of GWs can be expected, so that these systems could be very interesting for the above mentioned ground-based and space detectors;

- the amplitudes of the strongest GW signals are expected to be roughly an order of magnitude smaller than LISA's instrumental noise.

We investigate the GW emission by binary systems, in the extreme mass ratio limit, by the quadrupole approximation, considering orbits affected by both nutation and precession effects, taking into account also gravitomagnetic terms in the weak field approximation of the metric. We will see that gravitational waves are emitted with a "peculiar" signature related to the orbital features: such a signature may be a "burst" wave-form with a maximum in correspondence to the periastron distance or a modulated waveform, according to the orbit stability. Here we face this problem discussing in detail the dynamics of such a phenomenon which could greatly improve the statistics of possible GW sources.

Besides, we give estimates of the distributions of these sources and their parameters. It is worth noticing that the captures occur when objects, in the dense stellar cusp surrounding a galactic $\mathrm{MBH}$, undergo a close encounter, so that the trajectory becomes tight enough that orbital decay through emission of GWs dominates the subsequent evolution. According to Refs. [74, 75]), for a typical capture, the initial orbital eccentricity is extremely large (typically $\left.1-e \sim 10^{-6}-10^{-3}\right)$ and the initial pericenter distance very small ( $r_{\mathrm{p}} \sim 8-100 M$, where $M$ is the MBH mass [115]. The subsequent orbital evolution may (very roughly) be divided into three stages. In the first and longest stage the orbit is extremely eccentric, and GWs are emitted in short "pulses" during pericenter passages. These GW pulses slowly remove energy and angular momentum from the system, and the orbit gradually shrinks and circularizes. After $\sim 10^{3}-10^{8}$ years (depending on the two masses and the initial eccentricity) the evolution enters its second stage, where the orbit is sufficiently circular: the emission can be viewed as continuous. Finally, as the object reaches the last stable orbit, the adiabatic inspiral transits to a direct plunge, and the GW signal cuts off. Radiation reaction quickly circularizes the orbit over the inspiral phase; however, initial eccentricities are large enough that a substantial fraction of captures will maintain high eccentricity until the final plunge. It has been estimated [75] that about half of the captures will plunge with eccentricity $e>0.2$. While individually-resolvable captures will mostly be detectable during the last $\sim 1-100$ yrs of the second stage (depending on the stellar mass $m$ and the MBH mass), radiation emitted during the first stage will contribute significantly to the confusion background. As we shall see, the above scenario is heavily modified since the gravitomagnetic effects play a crucial role in modifying the orbital shapes that are far from being simply circular or elliptic and no longer closed.

\section{A. Gravitational Waves Amplitude Considering Orbits with Gravitomagnetic Corrections}

Direct signatures of gravitational radiation are given by $\mathrm{GW}$-amplitudes and waveforms. In other words, the identification of a GW signal is strictly related to the accurate selection of the waveform shape by interferometers or any possible detection tool. Such an achievement could give information on the nature of the GW source, on the propagating medium, and, in principle, on the gravitational theory producing such a radiation [116].

Considering the formulas of previous Section, the GWamplitude can be evaluated by

$$
h^{j k}(t, R)=\frac{2 G}{R c^{4}} \ddot{Q}^{j k}
$$

$R$ being the distance between the source and the observer and, due to the above polarizations, $\{j, k\}=1,2$. 


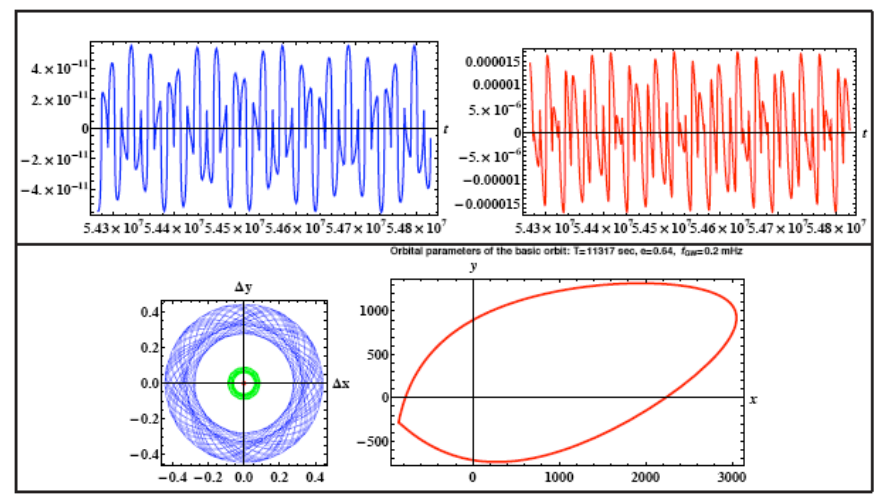

Fig. (18). Plots of $z_{N O}(t)$ (left upper panel) and $z_{\text {Grav }}(t)$ (right upper panel). It is interesting to see the differences of about five orders of magnitude between the two plots. At the beginning, the effect is very small but, orbit by orbit, it grows and, for a suitable interval of coordinated time, the effect cannot be neglected (see the left bottom panel in which the differences in $x$ and $y$, starting from the initial orbits up to the last ones, by steps of about 1500 orbits, are reported). The internal red circle represents the beginning, the middle one is the intermediate situation (green) and the blue one is the final result of the correlation between $\Delta x$ versus $\Delta y$, being $\Delta x=x_{\text {Grav }}-x_{N O}$ and $\Delta y=y_{\text {Grav }}-y_{N O}$. On the bottom right, it is shown the basic orbit.

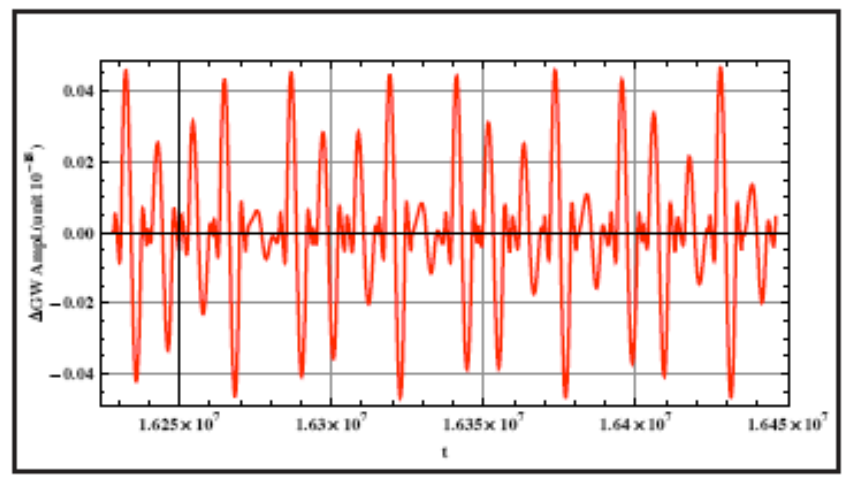

Fig. (19). Plot of the differences of total gravitational waveform $h$, with and without the gravitomagnetic orbital correction for a neutron star of $1.4 M_{\odot}$ orbiting around a MBH . The waveform has been computed at the Earth-distance from SgrA * (the central Galactic Black Hole). The example we are showing has been obtained solving the systems for the following parameters and initial conditions: $\mu \approx 1.4 M_{\odot}, r_{0}$, $E=0.95, \phi_{0}=0, \theta_{0}=\frac{\pi}{2}, \dot{\theta}_{0}=0, \phi_{0}=-\frac{1}{10} \dot{r}_{0}$ and $\dot{r}_{0}=-\frac{1}{100}$. It is worth noticing that frequency modulation gives cumulative effects after suitable long times.

From Eq. (9.1), it is straightforward to show that, for a binary system where $m \ll M$ and orbits have gravitomagnetic corrections, the Cartesian components of GW-amplitude are

$$
\begin{aligned}
& h^{x x}=2 \mu\left[\left(3 \cos ^{2} \phi_{\sin ^{2}}{ }^{2}-1\right) \dot{r}^{2}+6 r\left(\dot{\theta}_{\cos }{ }^{2} \phi \sin 2 \theta\right.\right. \\
& \left.-\phi_{\sin ^{2}}{ }^{2} \sin 2 \phi\right) \dot{r}+r\left(\left(3 \cos ^{2} \phi_{\sin ^{2}}{ }^{2} \theta-1\right) \ddot{r}\right. \\
& +3 r\left(\dot{\theta}^{2} \cos 2 \theta_{\cos }^{2} \phi-\phi \dot{\theta} \sin 2 \theta \sin 2 \phi\right. \\
& -\sin \theta\left(\sin \theta\left(\phi^{2} \cos 2 \phi+\phi \cos \phi \sin \phi\right)-\ddot{\theta} \cos \theta_{\left.\left.\left.\left.\cos ^{2} \phi\right)\right)\right)\right],}\right.
\end{aligned}
$$

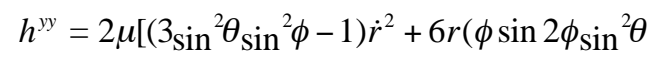

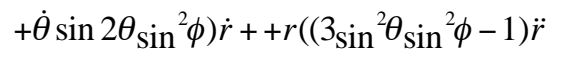

$$
\begin{aligned}
& +3 r\left(\dot{\theta}^{2} \cos 2 \theta_{\sin ^{2} \phi+\phi \dot{\theta} \sin 2 \theta \sin 2 \phi}\right.
\end{aligned}
$$

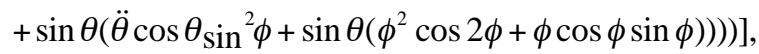

$$
\begin{aligned}
& h^{x y}=h^{y x}=3 \mu\left[\cos 2 \phi \sin \theta(4 \dot{\theta} \phi \cos \theta+\phi \sin \theta) r^{2}\right. \\
& +2 \dot{r}\left(2 \phi \cos 2 \phi_{\sin ^{2}}{ }^{2} \theta+\dot{\theta} \sin 2 \theta \sin 2 \phi\right) r \\
& +\frac{1}{2} \sin 2 \varphi\left(2 \ddot{r}_{\sin ^{2}} \theta+r\left(2 \dot{\theta}^{2} \cos 2 \theta-4 \dot{\varphi}^{2} \sin ^{2} \theta\right.\right. \\
& +\ddot{\theta} \sin 2 \theta)) r+\dot{r}^{2} \sin (\theta \sin 2 \phi],
\end{aligned}
$$

where we are assuming geometrized units. The above formulas have been obtained from Eqs. (4.27), (4.28), (4.29). The gravitomagnetic corrections give rise to signatures on the GW-amplitudes that, in the standard Newtonian orbital motion, are not present (see for example [32, 33]). On the other hand, as discussed in the Introduction, such corrections cannot be discarded in peculiar situations as dense stellar clusters or in the vicinity of galaxy central regions. We are going to evaluate these quantities and results are shown in Figs. (18, 19, 20, 21). 


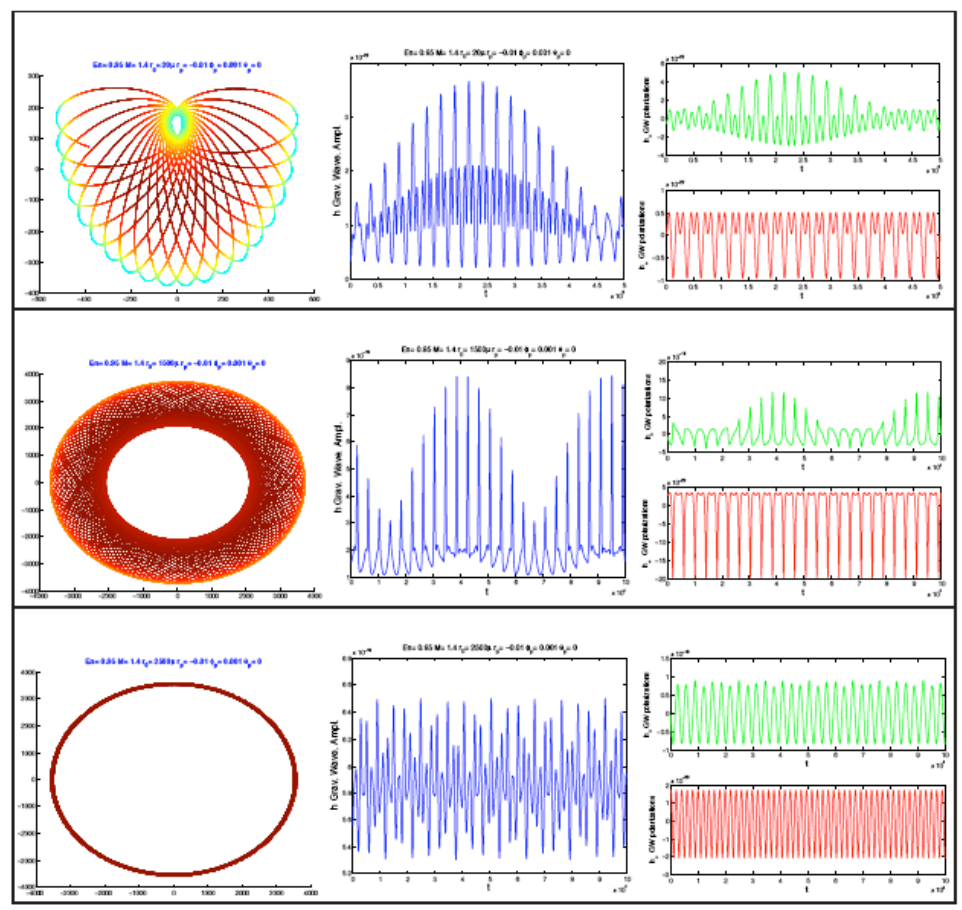

Fig. (20). Plots along the panel lines from left to right of field velocities along the axes of maximum covariances, total gravitational emission waveform $h$ and gravitational waveform polarizations $h_{+}$and $h_{x}$ for a neutron star of $1.4 M_{*}$. The waveform has been computed for the Earth-distance from Sagittarius A (the central Galactic Black Hole SgrA ${ }^{*}$ ). The plots we are showing have been obtained solving the system for the following parameters and initial conditions: $\mu \approx 1.4 M_{\odot}, E=0.95, \varphi_{0}=0, \theta_{0}=\frac{\pi}{2}, \dot{\theta}_{0}=0, \dot{\varphi}_{0}=-\frac{1}{10} \dot{r}_{0}$ and $\dot{r}_{0}=-\frac{1}{100}$. From top to bottom of the panels, the orbital radius is $r_{0}=20 \mu, 1500 \mu, 2500 \mu$. See also Table $\mathbf{I}$.

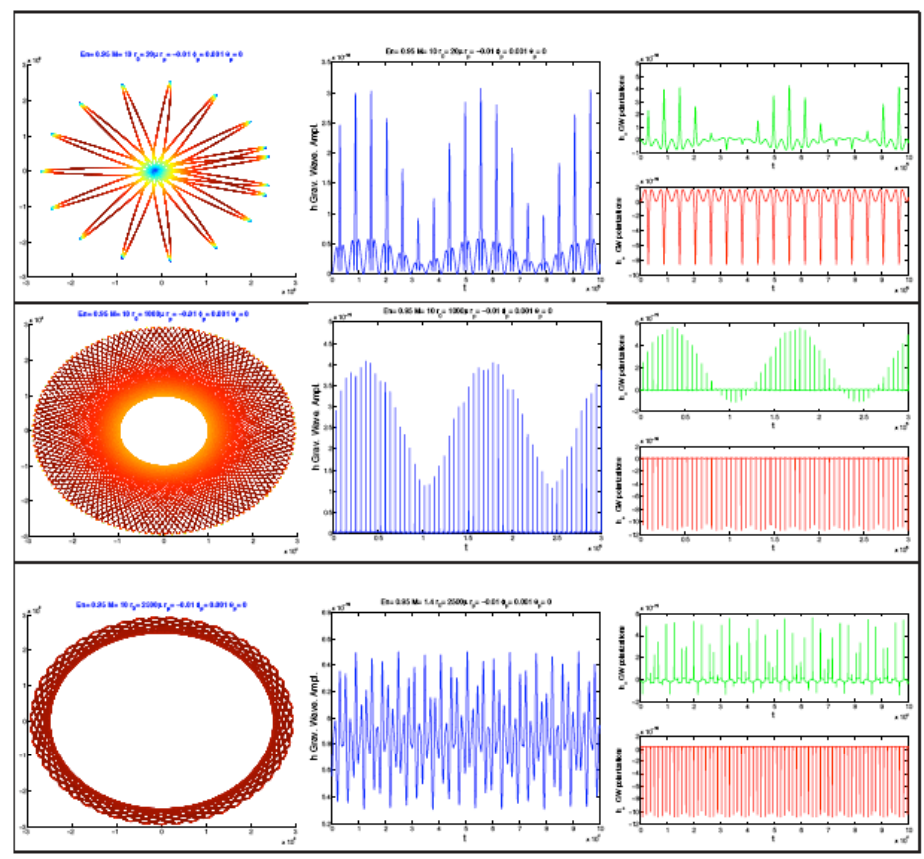

Fig. (21). Plots along the panel lines from left to right of field velocities along the axes of maximum covariances, total gravitational emission waveform $h$ and gravitational waveform polarizations $h_{+}$and $h_{x}$ for a Black Hole (BH) of $10 M_{\odot}$. The waveform has been computed for the Earth-distance to SgrA ${ }^{*}$. The plots we are showing have been obtained solving the system for the following parameters and initial conditions: $\mu \approx 10 M_{\odot}, E=0.95, \dot{\theta}_{0}=0 \phi_{0}=0, \theta_{0}=\frac{\pi}{2}, \dot{\theta}_{0}=0, \phi_{0}=-\frac{1}{10} \dot{r}_{0}$ and $\dot{r}_{0}=-\frac{1}{100}$. From top to bottom of the panels, the orbital radius is $r_{0}=20 \mu, 1000 \mu, 2500 \mu$. See also Table $\mathbf{I}$. 
Table 3. GW-amplitudes and Frequencies as Function of Eccentricity $e$, Reduced Mass $\mu$, Orbital Radius $r_{0}$ for the two Cases of Fiducial Stellar Objects $m \simeq 1.4 M_{\odot}$ and $m \simeq 10 M_{\odot}$ Orbiting Around a MBH of Mass $M \simeq 3 \times 10^{6} M_{\odot}$

\begin{tabular}{||c|c|c|c|c|c|c|c|c|c|c||}
\hline \multicolumn{7}{|c|}{$1.4 M_{\odot}$} & \multicolumn{3}{|c|}{$10 M_{\odot}$} \\
\hline$\frac{r_{0}}{\mu}$ & $e$ & $f(m H z)$ & $h$ & $h_{+}$ & $h_{\times}$ & $e$ & $f(m H z)$ & $h$ & $h_{+}$ & $h_{\times}$ \\
\hline \hline 20 & 0.91 & $7.7 \cdot 10^{-2}$ & $2.0 \cdot 10^{-22}$ & $5.1 \cdot 10^{-23}$ & $5.1 \cdot 10^{-22}$ & 0.98 & $3.2 \cdot 10^{-2}$ & $1.5 \cdot 10^{-18}$ & $1.6 \cdot 10^{-19}$ & $4.3 \cdot 10^{-18}$ \\
200 & 0.79 & $1.1 \cdot 10^{-1}$ & $1.2 \cdot 10^{-20}$ & $2.2 \cdot 10^{-21}$ & $3.1 \cdot 10^{-20}$ & 0.87 & $9.2 \cdot 10^{-2}$ & $1.5 \cdot 10^{-16}$ & $2.5 \cdot 10^{-18}$ & $4.1 \cdot 10^{-16}$ \\
500 & 0.64 & $1.4 \cdot 10^{-1}$ & $6.9 \cdot 10^{-20}$ & $8.7 \cdot 10^{-21}$ & $1.7 \cdot 10^{-19}$ & 0.71 & $1.4 \cdot 10^{-1}$ & $8.5 \cdot 10^{-16}$ & $7.0 \cdot 10^{-18}$ & $2.4 \cdot 10^{-15}$ \\
1000 & 0.44 & $1.9 \cdot 10^{-1}$ & $2.6 \cdot 10^{-19}$ & $6.4 \cdot 10^{-20}$ & $6.4 \cdot 10^{-19}$ & 0.49 & $1.9 \cdot 10^{-1}$ & $2.0 \cdot 10^{-15}$ & $1.6 \cdot 10^{-17}$ & $5.6 \cdot 10^{-15}$ \\
1500 & 0.28 & $2.3 \cdot 10^{-1}$ & $4.8 \cdot 10^{-19}$ & $3.6 \cdot 10^{-20}$ & $1.2 \cdot 10^{-18}$ & 0.32 & $2.3 \cdot 10^{-1}$ & $2.7 \cdot 10^{-15}$ & $2.5 \cdot 10^{-17}$ & $7.4 \cdot 10^{-15}$ \\
2000 & 0.14 & $2.7 \cdot 10^{-1}$ & $5.9 \cdot 10^{-19}$ & $4.9 \cdot 10^{-20}$ & $1.3 \cdot 10^{-18}$ & 0.19 & $2.6 \cdot 10^{-1}$ & $2.8 \cdot 10^{-15}$ & $3.3 \cdot 10^{-17}$ & $7.6 \cdot 10^{-15}$ \\
2500 & 0.01 & $3.1 \cdot 10^{-1}$ & $5.9 \cdot 10^{-19}$ & $1.7 \cdot 10^{-20}$ & $9.2 \cdot 10^{-19}$ & 0.08 & $2.9 \cdot 10^{-1}$ & $2.1 \cdot 10^{-15}$ & $4.0 \cdot 10^{-17}$ & $5.6 \cdot 10^{-15}$ \\
\hline
\end{tabular}

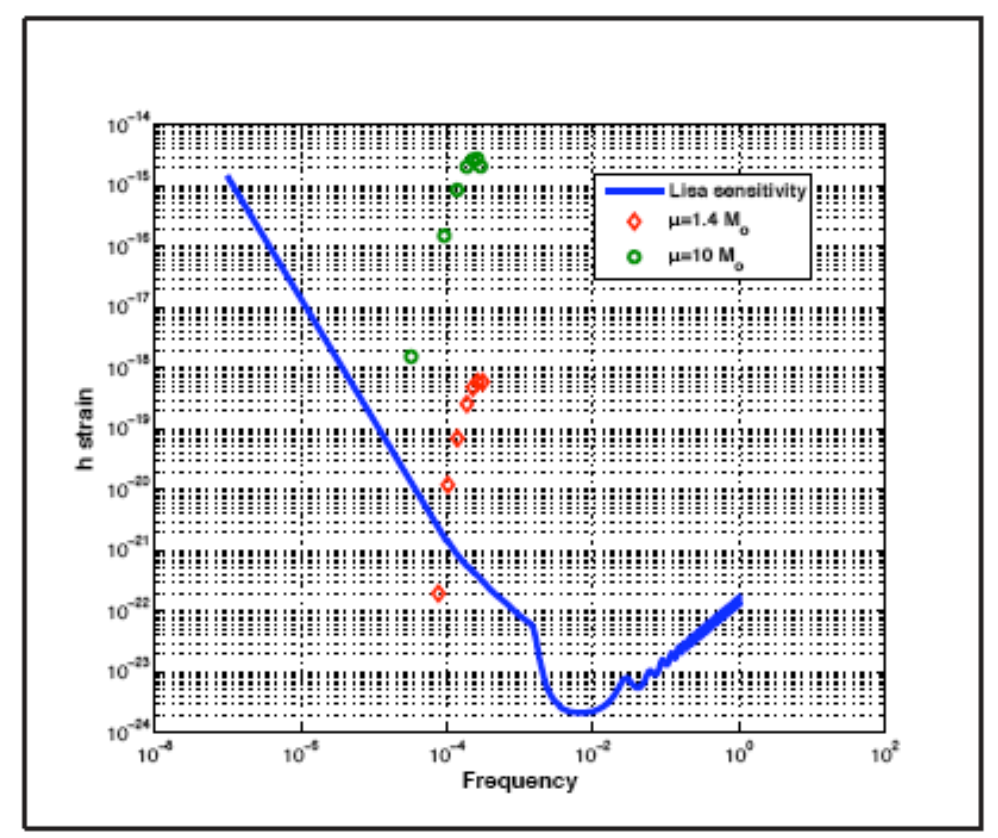

Fig. (22). Plot of estimated mean values of GW-emission in terms of strain $h$ for two binary sources at the Galactic Center SgrA* with reduced mass $\mu \approx 1.4 M_{\odot}$ (red diamonds) and $\mu \approx 10 M_{\odot}$ (green circles). The blue line is the foreseen LISA sensitivity curve. The waveforms have been computed for the Earth-distance to $\operatorname{SgrA}^{*}$. The examples we are showing have been obtained solving the systems for the parameters and initial conditions reported in Figs. 20, 21 and in Table $\mathbf{I}$.

\section{B. Numerical Results}

Now we have all the ingredients to estimate the effects of gravitomagnetic corrections on the GW-radiation. Calculations have been performed in geometrized units in order to evaluate better the relative corrections in absence of gravitomagnetism. For the numerical simulations, we have assumed the fiducial systems constituted by a $m=1.4 M_{\odot}$ neutron star or $m=10 M_{\odot}$ massive stellar object orbiting around a $\mathrm{MBH} \quad M \simeq 3 \times 10^{6} M_{\odot}$ as $\mathrm{SgrA}^{*}$. In the extreme mass-ratio limit, this means that we can consider $\mu=\frac{m M}{m+M}$ of about $\mu \approx 1.4 M_{\odot}$ and $\mu \approx 10 M_{\odot}$. The computations have been performed starting with orbital radii measured in the mass unit and scaling the distance according to the values shown in Table $\mathbf{I}$. As it is possible to see in Table I, starting from $r_{0}=20 \mu$ up to $2500 \mu$, the orbital eccentricity $\mathbf{e}=\frac{r_{\max }-r_{\min }}{r_{\max }+r_{\min }}$ evolves towards a circular orbit. In Table I, the GW-frequencies, in $m H z$, as well as the $h$ amplitude strains and the two polarizations $h_{+}$and $h_{\times}$are shown. The values are the mean values of the $\mathrm{GW} h$ amplitude strains $\left(h=\frac{h_{\max }+h_{\min }}{2}\right)$ and the maxima of the polarization waves (see Figs. 20 and 21). In Fig. (22), the fiducial LISA sensitivity curve is shown [13] considering the confusion noise produced by White Dwarf binaries (blue 
curve). We show also the $h$ amplitudes (red diamond and green circles for $\mu \approx 1.4 M_{\odot}$ and $\approx 10 M_{\odot}$ respectively). It is worth noticing that, due to very high Signal to Noise Ratio, the binary systems which we are considering result extremely interesting, in terms of probability detection, for the LISA interferometer (see Fig. 22).

\section{RATE AND EVENT NUMBER ESTIMATIONS IN DENSE STELLAR SYSTEMS}

At this point, it is important to give some estimates of the number of events where gravitomagnetic effects could be a signature for orbital motion and gravitational radiation. From the GW emission point of view, close orbital encounters, collisions and tidal interactions have to be dealt on average if we want to investigate the gravitational radiation in a dense stellar system. On the other hand, dense stellar regions are the favored target for LISA interferometer [111] so it is extremely useful to provide suitable numbers before its launching.

To this end, it is worth giving the stellar encounter rate producing GWs in astrophysical systems like dense globular clusters or the Galactic Center. In general, stars are approximated as point masses. However, in dense regions of stellar systems, a star can pass so close to another that they raise tidal forces which dissipate their relative orbital kinetic energy and the Newtonian mechanics or the weak field limit of GR cannot be adopted as good approximations. In some cases, the loss of energy can be so large that stars form binary (the situation which we have considered here) or multiple systems; in other cases, the stars collide and coalesce into a single star; finally stars can exchange gravitational interaction in non-returning encounters.

To investigate and parameterize all these effects, one has to compute the collision time $t_{\text {coll }}$, where $1 / t_{\text {coll }}$ is the collision rate, that is, the average number of physical collisions that a given star suffers per unit time. As a rough approximation, one can restrict to stellar clusters in which all stars have the same mass $m$.

Let us consider an encounter with initial relative velocity $\mathbf{v}_{0}$ and impact parameter $b$. The angular momentum per unit mass of the reduced particle is $L=b v_{0}$. At the distance of closest approach, which we denote by $r_{\text {coll }}$, the radial velocity must be zero, and hence the angular momentum is $L=r_{\text {coll }} v_{\text {max }}$, where $v_{\max }$ is the relative speed at $r_{\text {coll }}$. It is easy to show that [25]

$$
b^{2}=r_{\text {coll }}^{2}+\frac{4 G m r_{\text {coll }}}{v_{0}^{2}} .
$$

If we set $r_{\text {coll }}$ equal to the sum of the radii of the two stars, a collision will occur if the impact parameter is less than the value of $b$, as determined by Eq. (10.1).

The function $f\left(\mathbf{v}_{a}\right) d^{3} \mathbf{v}_{a}$ gives the number of stars per unit volume with velocities in the range $\mathbf{v}_{a}+d^{3} \mathbf{v}_{a}$. The number of encounters per unit time with impact parameter less than $b$, which are suffered by a given star, is $f\left(\mathbf{v}_{a}\right) d^{3} \mathbf{v}_{a}$ times the volume of the annulus with radius $b$ and length $v_{0}$, that is,

$$
\int f\left(\mathbf{v}_{a}\right) \pi b^{2} v_{0} d^{3} \mathbf{v}_{a},
$$

where $v_{0}=\left|\mathbf{v}-\mathbf{v}_{a}\right|$ and $\mathbf{v}$ is the velocity of the considered star. The quantity in Eq. (10.2) is equal to $1 / t_{\text {coll }}$ for a star with velocity $\mathbf{v}$ : to obtain the mean value of $1 / t_{\text {coll }}$, we average over $\mathbf{v}$ by multiplying (10.2) by $f(\mathbf{v}) / v$, where $v=\int f(\mathbf{v}) d^{3} \mathbf{v}$ is the number density of stars and the integration is over $d^{3} \mathbf{v}$. Thus

$$
\begin{aligned}
& \frac{1}{t_{\text {coll }}}=\frac{v}{8 \pi^{2} \sigma^{6}} \int e^{-\left(v^{2}+v_{a}^{2}\right) / 2 \sigma^{2}} \times \\
& \left(r_{\text {coll }}\left|\mathbf{v}-\mathbf{v}_{a}\right|+\frac{4 G m r_{\text {coll }}}{\left|\mathbf{v}-\mathbf{v}_{a}\right|}\right) d^{3} \mathbf{v} d^{3} \mathbf{v}_{a} .
\end{aligned}
$$

Replacing the variable $\mathbf{v}_{a}$ by $\mathbf{V}=\mathbf{v}-\mathbf{v}_{a}$, the argument of the exponential is then $-\left[\left(\mathbf{v}-\frac{1}{2} \mathbf{V}\right)^{2}+\frac{1}{4} V^{2}\right] / \sigma^{2}$, and if we replace the variable $\mathbf{v}$ by $\mathbf{v}_{c m}=\mathbf{v}-\frac{1}{2} \mathbf{V}$ (the center of mass velocity), then one has

$$
\frac{1}{t_{\text {coll }}}=\frac{v}{8 \pi^{2} \sigma^{6}} \int e^{-\left(v_{c m}^{2}+V^{2}\right) / 2 \sigma^{2}}\left(r_{\text {coll }} V+\frac{4 G m r_{\text {coll }}}{V}\right) d V .
$$

The integral over $\mathbf{v}_{c m}$ is given by

$$
\int e^{-v_{c m}^{2} / \sigma^{2}} d^{3} \mathbf{v}_{c m}=\pi^{3 / 2} \sigma^{3} .
$$

Thus

$$
\frac{1}{t_{\text {coll }}}=\frac{\pi^{1 / 2} v}{2 \sigma^{3}} \int_{\infty}^{0} e^{-V^{2} / 4 \sigma^{2}}\left(r_{\text {coll }}^{2} V^{3}+4 G m V r_{\text {coll }}\right) d V
$$

The integrals can be easily calculated and then we find

$$
\frac{1}{t_{\text {coll }}}=4 \sqrt{\pi} v \sigma r_{\text {coll }}^{2}+\frac{4 \sqrt{\pi} v G m r_{\text {coll }}}{\sigma} .
$$

The first term of this result can be derived from the kinetic theory. The rate of interaction is $v \Sigma\langle V\rangle$, where $\Sigma$ is the cross-section and $\langle V\rangle$ is the mean relative speed. Substituting $\Sigma=\pi r_{\text {coll }}^{2}$ and $\langle V\rangle=4 \sigma / \sqrt{\pi} \quad$ (which is appropriate for a Maxwellian distribution with dispersion $\sigma$ ) we recover the first term of (10.7). The second term represents the enhancement in the collision rate by 
gravitational focusing, that is, the deflection of trajectories by the gravitational attraction of the two stars.

If $r_{*}$ is the stellar radius, we may set $r_{\text {coll }}=2 r_{*}$. It is convenient to introduce the escape speed from stellar surface, $v_{*}=\sqrt{\frac{2 G m}{r_{*}}}$, and to rewrite Eq. (10.7) as

$$
\Gamma=\frac{1}{t_{\text {coll }}}=16 \sqrt{\pi} v \sigma r_{*}^{2}\left(1+\frac{v_{*}^{2}}{4 \sigma^{2}}\right)=16 \sqrt{\pi} v \sigma r_{*}^{2}(1+\Theta)
$$

where

$$
\Theta=\frac{v_{*}^{2}}{4 \sigma^{2}}=\frac{G m}{2 \sigma^{2} r_{*}}
$$

is the Safronov number [25]. In evaluating the rate, we are considering only those encounters producing gravitational waves, for example, in the LISA range, i.e. between $10^{-4}$ and $10^{-1} \mathrm{~Hz}$ (see e.g. [117]). Numerically, we have

$$
\begin{aligned}
& \Gamma \simeq 5.5 \times 10^{-10}\left(\frac{v}{10 \mathrm{kms}^{-1}}\right)\left(\frac{\sigma}{U A^{2}}\right)\left(\frac{10 p c}{R}\right)^{3}{\mathrm{y} r s^{-1}}^{-1} \\
& \Gamma<<1 \\
& \Gamma \simeq 5.5 \times 10^{-10}\left(\frac{M}{10^{5} \mathrm{M}}\right)^{2}\left(\frac{v}{10 \mathrm{kms}^{-1}}\right)\left(\frac{\sigma}{U A^{2}}\right) \times \\
& \left(\frac{10 p c}{R}\right)^{3} \mathrm{yrs}^{-1} \quad \Theta>>1
\end{aligned}
$$

If $\Theta>>1$, the energy dissipated exceeds the relative kinetic energy of the colliding stars, and the stars coalesce into a single star. This new star may, in turn, collide and merge with other stars, thereby becoming very massive. As its mass increases, the collision time is shorten and then there may be runaway coalescence leading to the formation of a few supermassive objects per clusters. If $\Theta<<1$, much of the mass in the colliding stars may be liberated and forming new stars or a single supermassive objects (see [118]). Both cases are interesting for LISA purposes.

Note that when one has the effects of quasi-collisions (where gravitomagnetic effects, in principle, cannot be discarded) in an encounter of two stars in which the minimal separation is several stellar radii, violent tides will raise on the surface of each star. The energy that excites the tides comes from the relative kinetic energy of the stars. This effect is important for $\Theta \gg 1$ since the loss of small amount of kinetic energy may leave the two stars with negative total energy, that is, as a bounded binary system. Successive pericenter passages will dissipates more energy by GW radiation, until the binary orbit is nearly circular with a negligible or null GW radiation emission.

Let us apply these considerations to the Galactic Center which can be modelled as a system of several compact stellar clusters, some of them similar to very compact globular clusters with high emission in X-rays [119].
For a typical globular cluster around the Galactic Center, the expected event rate is of the order of $2 \times 10^{-9} \mathrm{yrs}^{-1}$ which may be increased at least by a factor $\simeq 100$ if one considers the number of globular clusters in the whole Galaxy. If the stellar cluster at the Galactic Center is taken into account and assuming the total mass $M \simeq 3 \times 10^{6} \mathrm{M}_{\odot}$, the velocity dispersion $\sigma \simeq 150 \mathrm{~km} \mathrm{~s}^{-1}$ and the radius of the object $R \simeq 10 \mathrm{pc}$ (where $\Theta=4.3$ ), one expects to have $\simeq 10^{-5}$ open orbit encounters per year. On the other hand, if a cluster with total mass $M \simeq 10^{6} \mathrm{M}_{\odot}, \sigma \approx 150 \mathrm{~km} \mathrm{~s}^{-1}$ and $R \approx 0.1 \mathrm{pc}$ is considered, an event rate number of the order of unity per year is obtained. These values could be realistically achieved by data coming from the forthcoming space interferometer LISA. As a secondary effect, the above wave-forms could constitute the "signature" to classify the different stellar encounters thanks to the differences of the shapes (see Figs. 20 and 21).

\section{DISCUSSION, CONCLUSIONS PERSPECTIVES}

AND

We have considered the two-body problem in Newtonian and relativistic theory of orbits in view of characterizing the gravitational radiation, starting from the motion of the sources. We have reported several results concerning the equations of motion, and the associated Lagrangian formulation, of compact binary systems. These equations are necessary when constructing the theoretical templates for searching and analyzing the GW signals from inspiralling compact binaries in VIRGO-LIGO and LISA type experiments. Considering the two-body problem, we mean the problem of the dynamics of two structureless, nonspinning point-particles, characterized by solely two mass parameters $m_{1}$ and $m_{2}$, moving under their mutual, purely gravitational interaction. Surely this problem, because of its conceptual simplicity, is among the most interesting ones to be solved within any theory of gravity. Actually, there are two aspects of the problem: the first sub-problem consists into obtaining the equation of the binary motion, the second is to find the (hopefully exact) solution of that equation. We referred to the equation of motion as the explicit expression of the acceleration of each of the particles in terms of their positions and velocities. It is well known that in Newtonian gravity, the first of these sub-problems is trivial, as one can easily write down the equation of motion for a system of $N$ particles, while the second one is difficult, except in the twobody case $N=2$, which represents, in fact, the only situation amenable to an exact treatment of the solution. In GR, even writing down the equations of motion in the simplest case $N=2$ is difficult. Unlike in Newton's theory, it is impossible to express the acceleration by means of the positions and velocities, in a way which would be valid within the exact theory. Therefore we are obliged to resort to approximation methods. Let us feel reassured that plaguing the exact theory of GR with approximation methods is not a shame. It is fair to say that many of the great successes of this theory, when confronted to experiments and observations, have been obtained thanks to approximation 
methods. Furthermore, the beautiful internal wheels of GR also show up when using approximation methods, which often deserve some theoretical interest in their own, as they require interesting mathematical techniques. Here we have investigated the equation of the binary motion in the postNewtonian approximation, i.e. as a formal expansion when the velocity of light $c$ tends to infinity. As a consequence of the equivalence principle, which is incorporated by hand in Newton's theory and constitutes the fundamental basis of GR, the acceleration of particlel should not depend on $m_{1}$ (nor on its internal structure), in the test-mass limit where the mass $m_{1}$ is much smaller than $m_{2}$. This is, of course, satisfied by the Newtonian acceleration, which is independent of $m_{1}$, but this leaves the possibility that the acceleration of the particle1, in higher approximations, does depend on $m_{1}$, via the so-called self-forces, which vanish in the test-mass limit. Indeed, this is what happens in the postNewtonian and gravitomagnetic corrections, which show explicitly many terms proportional to (powers of) $m_{1}$. Though the approximations and corrections to the orbits are really a consequence of GR, they should be interpreted using the common-sense language of Newton. That is, having chosen a convenient general-relativistic (Cartesian) coordinate system, like the harmonic coordinate system adopted above, we have express the results in terms of the coordinate positions, velocities and accelerations of the bodies. Then, the trajectories of the particles can be viewed as taking place in the absolute Euclidean space of Newton, and their (coordinate) velocities as being defined with respect to absolute time. Not only this interpretation is the most satisfactory one from a conceptual point of view, but it represents also the most convenient path for comparing the theoretical predictions and the observations. For instance, the Solar System dynamics at the first post-Newtonian level is defined, following a recent resolution of the International Astronomical Union, in a harmonic coordinate system, the Geocentric Reference System (GRS), with respect to which one considers the absolute motion of the planets and satellites. But because the equations come from GR, they are endowed with the following properties, which make them truly relativistic.

- The one-body problem in GR corresponds to the Schwarzschild solution, so the equations possess the correct perturbative limit, that given by the geodesics of the Schwarzschild metric, when the mass of one of the bodies tends to zero.

- Because GR admits the Poincaré group as a global symmetry (in the case of asymptotically flat space-times), the harmonic-coordinate equations of motion stay invariant when we perform a global Lorentz transformation.

- Since the particles emit gravitational radiation there are some terms in the equations which are associated with radiation reaction. These terms appear at the order $2.5 \mathrm{PN}$ or $c^{-} 5$ that we discarded in our discussion (where $5=2 s+1$, $s=2$ being the helicity of the graviton). They correspond to an odd- order PN correction, which does not stay invariant in a time reversal. By contrast, the even-orders, as 1PN, correspond to a dynamics which is conservative.

- GR is a non-linear theory (even in vacuum), and some part of the gravitational radiation which was emitted by the particles in the past scatters off the static gravitational field generated by the rest-masses of the particles, or interacts gravitationally with itself.

From all these considerations, the post-Newtonian equations were also obtained, for the motion of the centers of mass of extended bodies, using a technique that can be qualified as more physical than the surface-integral method, as it takes explicitly into account the structure of the bodies.

Particularly interesting is considering gravitomagnetic effects in the geodesic motion. In particular, one can consider the orbital effects of higher-order terms in $v / c$ which is the main difference with respect to the standard approach to the gravitomagnetism. Such terms are often discarded but, as we have shown, they could give rise to interesting phenomena in tight binding systems as binary systems of evolved objects (neutron stars or black holes). They could be important for objects falling toward extremely massive black holes as those seated in the galactic centers $[74,75]$. The leading parameter for such correction is the ratio $v / c$ which, in several physical cases cannot be simply discarded. For a detailed discussion see for example [120123]. A part the standard periastron precession effects, such terms induce nutations and are capable of affecting the stability basin of the orbital phase space. As shown, the global structure of such a basin is extremely sensitive to the initial angular velocities, the initial energy and mass conditions which can determine possible transitions to chaotic behaviors. Detailed studies on the transition to chaos could greatly aid in gravitational wave detections in order to determine the shape, the spectrum and the intensity of the waves (for a discussion see [124, 125]).

In the second part of this review, we have summarized many of the most important topics in the theory of GWs. Linearized theory as described in is adequate to describe the propagation of GWs and to model the interaction of GWs with our detectors. A variety of formalisms have been developed.

-Newtonian theory The emission of gravitational waves from stellar encounters in Newtonian regime interacting on elliptical, hyperbolic and parabolic orbits is studied in the quadrupole approximation. Analytical expressions are then derived for the gravitational wave luminosity, the total energy output and gravitational radiation amplitude produced in tight impacts where two massive objects closely interact at an impact distance of $1 A U$.

-Post-Newtonian theory. PN theory is one of the most important of these formalisms, particularly for modeling binary systems. Roughly speaking, PN theory analyzes sources using an iterated expansion in two variables: The "gravitational potential", $\Phi \sim M / r$, where $M$ is a mass scale and $r$ characterizes the distance from the source; and velocities of internal motion, $v$. (In linearized theory, we assume $\Phi$ is small but place no constraints on $v$.) Newtonian gravity emerges as the first term in the expansion, and higher order corrections are found as the 
expansion is iterated to ever higher order. Our derivation of the quadrupole formula gives the leading order term in the PN expansion of the emitted radiation. See [126] and references therein for a comprehensive introduction to and explication of this subject.

-Gravitomagnetic corrections. The gravitomagnetic effect could give rise to interesting phenomena in tight binding systems such as binaries of evolved objects (NSs or $\mathrm{BHs})$. The effects reveal particularly interesting if $\underline{v}$ is in the range $\left(10^{-1} \div 10^{-3}\right) c$. They could be important for $C_{\text {objects }}$ captured and falling toward extremely massive black holes such as those at the Galactic Center. Gravitomagnetic orbital corrections, after long integration time, induce precession and nutation and then modification on the wave-form. In principle, GW emission could present signatures of gravitomagnetic corrections after suitable integration times in particular for the on going LISA space laser interferometric $\mathrm{GW}$ antenna.

To conclude, Henri Poincaré [127] once remarked that real problems can never be classified as solved or unsolved ones, but that they are always more and less solved. This remark applies particularly well to the problem of motion, which has had chequered history. Even the Newtonian problem of motion, which appeared to well understood after the development of the powerful methods of classical mechanics [128] embarked on an entirely new career after work of Poincaré which has led to many further developments (see [129, 130]). The Einsteinian problem of motion has not even reached a classical stage where the basic problems appear as well understood. At first sight the best developed approximation method in GR, the PN one, would seem to constitute such classical stage, but the literature on the PN problem of motion is full of repetitions, errors or ambiguities. We was to conclude this review by giving a list of issues that need to be clarified. We renounced this project because, if one wishes to look at the work done with a critical eye, nearly all aspects of the problem of motion and GWs need to be thoroughly re-investigates for mathematical, physical or conceptual reasons; so that the list of open problems would, consistent with the remark of Poincaré. One thing is certain: the problem of motion and GWs is no longer a purely theoretical problem, tanks to the improvement in the precision of positions measurements in the solar systems, and to the discovery of the binary pulsar $1913+16$ which is a relativistic laboratory; the problem has become an important tool of modern astrophysics. It is therefore of some urgency, not only to complete and unify the work already done, but also to develop new approaches in order to aim both formal and conceptual clarification of the basic issues, and to obtain more accurate explicit results.

\section{ACKNOWLEDGMENTS}

I thank S. Capozziello and L. Milano for fruitful discussions and for the useful suggestions on the topics of this review.

\section{REFERENCES}

[1] Einstein A. Approximative Integration of the Field Equations of Gravitation. Preuss Akad Wiss Berlin, Sitzber 1916; 39: 688-96. (a) Einstein A, Infeld L. The Gravitational Equations and the Problem of Motion. II. Ann Math 1940;41: 455. (b) Einstein A, Infeld L.

[14] Poisson E. The motion of point particles in curved space-time. Living Rev Rel 2004; 6: 3.

[15] Barack L, Lousto C. Perturbations of Schwarzschild black holes in the Lorenz gauge: Formulation and numerical implementation. Phys Rev D 2005; 72: 104026. (a) Barack L, Sago N. Gravitational self-force on a particle in circular orbit around a Schwarzschild black hole. Phys Rev D 2007; 75: 064021.

[16] Sigl G, Schnittman J, Buonanno A. Gravitational-wave background from compact objects embedded in active galactic nuclei accretion disks. Phys Rev D 2007;75: 024034.

[17] Cutler C, Apostolatos A, Bildstenet L, et al. The last three minutes: Issues in gravitational-wave measurements of coalescing compact binaries. Phys Rev Lett 1993; 70: 2984. (a) Damour T, Iyer B, Sathyaprakash B. Comparison of search templates for gravitational waves from binary inspiral. Phys Rev D 2001;63; 044023. (b)Damour T, Iyer B, Sathyaprakash B. Comparison of search templates for gravitational waves from binary inspiral: 3.5PN update. Phys Rev D 2002; 66: 027502. (c) Damour T, Iyer B, Sathyaprakash B. Gravitational waves from black hole binary inspiral and merger: The span of third post-Newtonian effectiveone-body templates. Phys Rev D 2003;67: 064028. (d) Buonanno A, Chen Y, Vallisneri M. Detecting gravitational waves from precessing binaries of spinning compact objects: Adiabatic limit. Phys Rev D 2003; 67, 104025. (e) Buonanno A, Chen Y, Vallisneri M. Erratum: Detecting gravitational waves from precessing binaries of spinning compact objects: Adiabatic limit Phys. Rev. D 2006;74: 029904. (f) Buonanno A, Chen Y, Vallisneri M. Detection template families for gravitational waves from the final stages of binary black-hole inspirals: Non spinning case. Phys Rev D 2003; 67: 024016. (g) Damour T, Iyer B, Jaranowski P, Sathyaprakash B. Gravitational waves from black hole binary inspiral and merger: The span of third post-Newtonian effectiveone-body templates. Phys Rev D 2003; 67: 064028. (h) Pan Y, Buonanno A, Chen Y, Vallisneri M. Physical template family for gravitational waves from precessing binaries of spinning compact objects: Application to single-spin binaries. Phys Rev D 2004; 69: 104017. 
[18] Damour T, Iyer BR, Sathyaprakash BS. Improved filters for gravitational waves from inspiralling compact binaries. Phys Rev D 1998;57: 885.

[19] Buonanno A, Damour T. Effective one-body approach to general relativistic two-body dynamics. Phys Rev D 1999; 59: 084006. (a) Buonanno A. and Damour T. Effective Proceedings of IX ${ }^{\text {th }}$ Marcel Grossmann Meeting (Rome, July 2000) Available from [grqc/0011052]. (b) Damour $\mathrm{T}$, Jaranowski $\mathrm{P}$, Schäfer $\mathrm{G}$. Determination of the last stable orbit for circular general relativistic binaries at the third post-Newtonian approximation. Phys Rev D 2000;62: 084011. (c) Damour T. Coalescence of two spinning black holes: An effective one-body approach. Phys Rev D 2001; 64: 124013. (d) Buonanno A, Chen Y, Damour T. Transition from inspiral to plunge in precessing binaries of spinning black holes. Phys Rev D 2006; 74: 104005.

[20] Finn LS. Detection, measurement, and gravitational radiation. Phys Rev D 1992; 46: 5236. (a) Finn LS, Chernoff DF. Observing binary inspiral in gravitational radiation: One interferometer. Phys Rev D 1993;47: 2198. (b) Flanagan ÉE, Hughes SA. Measuring gravitational waves from binary black hole coalescences. I. Signal to noise for inspiral, merger, and ringdown. Phys Rev D 1998; 57: 4535.

[21] Brady PR, Creighton T, Cutler C, Schutz BF. Searching for periodic sources with LIGO. Phys Rev D 1998;57: 2101. (a) Brady PR, Creighton T. Searching for periodic sources with LIGO. II. Hierarchical searches. Phys Rev D 2001;61: 082001. (b) Tinto M, Estabrook FB, and Armstrong JW. Time-delay interferometry for LISA. Phys Rev D 2002;65: 082003. (c) Krishnan B, Sinteset A, Papa A, et al. Hough transform search for continuous gravitational waves. Phys Rev D 2004;70: 082001. (d) Cutler C, Gholami I, Krishnan B. Improved stack-slide searches for gravitational-wave pulsars. Phys Rev D 2005;72: 042004. (e) Chatterji S, Lazzarini A, Stein L, et al. Coherent network analysis technique for discriminating gravitational-wave bursts from instrumental noise. Phys Rev D 2006; 74: 082005.

[22] Abbott B, Abbott R, Adhikari R, et al. (LIGO Scientific Collaboration), Search for gravitational waves from primordial black hole binary coalescences in the galactic halo. Phys Rev D 2005;72: 082002. (a) Abbott B, Abbott R, Adhikari R, et al. (LIGO Scientific Collaboration). Upper limits from the LIGO and TAMA detectors on the rate of gravitational-wave bursts. Phys Rev D 2005; 72: 122004 .

[23] Abbott B, Abbott R, Adhikari R, et al. (LIGO Scientific Collaboration). Coherent searches for periodic gravitational waves from unknown isolated sources and Scorpius X-1. Phys Rev D 2007; $76: 082001$

[24] Abbott B, Abbott R, Adhikari R, et al. (LIGO Scientific Collaboration). Upper Limits on a Stochastic Background of Gravitational Waves. Phys Rev Lett. 2005; 95: 221101. (a) Abbott $\mathrm{B}$, Abbott R, Adhikari R, et al. Searching for a stochastic background of gravitational waves with LIGO. Astrophys J 2007; 659: 918-930.

[25] Binney J, Tremaine S. Galactic Dynamics. Princeton New Jersey: Princeton University Press, 1987.

[26] Landau LD, Lifshits EM. The Classical Theory of Field. Oxford: Pergamon Press 1975.

[27] Genzel R, Hollenbach D, Townes CH. The nucleus of our Galaxy. Rep Prog Phys 1994; 57: 417.

[28] Sellgreen K, Hall DNB, Kleinmann SG, Scoville NZ. Radial velocities of late-type stars in the galactic center. Ap J 1987; 317: 881.

[29] Capozziello S, Iovane G. Probing the nature of compact dark object at the Galactic Center by gravitational lensing. Phys Lett A 1999; 259: 185.

[30] Ghez AM, Klein BL, Morris M, Becklin EE. High Proper-Motion Stars in the Vicinity of Sagittarius A*: Evidence for a Supermassive Black Hole at the Center of Our Galaxy. Ap J 1998; 509: 678.

[31] Genzel R, Thatte N, Krabbe A, Kroker H, Tacconi-Garman LE. The Dark Mass Concentration in the Central Parsec of the Milky Way*. Ap J 1996; 472: 153

[32] Capozziello S, De Laurentis M, De Paolis F, Ingrosso G, Nucita A. Gravitational waves from hyperbolic encounters. Mod Phys Lett A 2008; 23: 99.

[33] Capozziello S, De Laurentis M. Gravitational waves from stellar encounters. Astroparticle Phys 2008; 30: 105-112.
[34] Peters PC, Mathews J. Gravitational Radiation from Point Masses in a Keplerian Orbit. Phys Rev 1963; 131: 435.

[35] Peters PC. Gravitational Radiation and the Motion of Two Point Masses. Phys Rev 1964; 136: 1224.

[36] Schutz BF. Gravitational collapse of a shell of quantized matter. Class Quant Grav 1999; 16: 131.

[37] Kovacs SJ, Thorne KS. The Generation of Gravitational Waves. III. Derivation of Bremsstrahlung Formulae. Ap J 1977; 217: 252 .

[38] Misner CW, Thorne KS, Wheeler JA. Gravitation, Freeman, New. York (1973).

[39] Weinberg S. Gravitation and Cosmology. New York, USA: Wiley 1972.

[40] Wald RM. General relativity. Chicago: The University of Chicago Press 1983.

[41] Schutz B. A first course in general relativity. Cambridge: Cambridge University Press 1985.

[42] Carroll S. Spacetime and geometry: an introduction to general relativity. Addison-Wesly 2003.

[43] Maggiore M. Gravitational Waves: Theory and Experiments, Oxford University Press 2007.

[44] Thorne KS. Gravitational radiation, in 300 Years of Gravitation ed. by S.W. Hawking and W. Israel Cambridge: Cambridge University Press 1987.

[45] Allen B. Lectures at Les Houches School. (1996) Available from [gr-qc/9604033].

[46] Maggiore M. Gravitational Wave Experiments and Early Universe Cosmology. Phys Rep 2000; 331: 283.

[47] Cutler C, Thorne KS. An overview of gravitational-wave sources. Available from [gr-qc/0204090]

[48] Buonanno A. TASI. lectures on gravitational-wave from the early Universe. Available from [gr-qc/0303085].

[49] Flanagan ÉE, Hughes SA. The basics of gravitational-wave theory. New J Phys 2005; 7: 204.

[50] Thorne KS. Available from: http://elmer.caltech.edu/ph237/

[51] Smart WM. Textbook on Spherical Astronomy. Cambridge: Cambridge University Press 1977.

[52] Roy AE. Orbital Motion. 3rd ed. Adam Hilger, 1988.

[53] Ehlers J. Isolated systems in general relativity. Ann N Y Acad Sci 1980; 336: 279.

[54] Damour T, Deruelle N. Generla relativistic celestial mechenics of binary systems. I. The post-Newtonian motion. Annales de l'I.H.P., Section A 1985; 43:107-132.

[55] Dixon WG. Isolated gravitating systems in General Relativity. Ehlers J ed. North-Holland: Amsterdam 1979; p. 156.

[56] Breuer RA, Rudolph E. The force law for the dynamic two-body problem in the second post-Newtonian approximation of general relativity. Gen Rel Grav 1982; 14: 181.

[57] Spyrou N. Relativistic equations of motion of extended bodies. Gen Rel Grav 1978; 9:519.

[58] D'Eath PD. Interaction of two black holes in the slow-motion limit. Phys Rev D 1975; 12: 2183.

[59] Kates RE. Motion of an electrically or magnetically charged body with possibly strong internal gravity through external electromagnetic and gravitational fields. Phys Rev 1980;22: 1871.

[60] Bel L, Damour T, Deruelle N, Ibanezz J, Martin J. Gen Rel Gray 1981;13: 936.

[61] Damour T, Deruelle N, Piran T. Gravitational radiation. Eds, NothHolland: Amsterdam 1983; p. 59.

[62] Damour T, Deruelle N. General relativistic celestial mechanics of binary systems I. Phys Lett A 1981; 87: 81.

[63] Infeld L, Plebanski J. Motion and Relativity. Oxford, Pergamon 1960.

[64] Wagoner RV, Will C. M. Post-Newtonian gravitational radiation from orbiting point masses Astrophys J 1976; 210: 764.

[65] Capozziello S, De Laurentis M, Garufi F, Milano L. Relativistic orbits with gravitomagnetic corrections. Physica Scripta 2009; 79: 025901.

[66] Capozziello S, Corda C, De Laurentis M. Stochastic background of gravitational waves 'tuned' by $\mathrm{f}(\mathrm{R})$ gravity. Mod Phys Lett A 2007; 22; 15:1097. (a) Capozziello S, Corda C, De Laurentis M. Stochastic background of relic scalar gravitational waves from scalar-tensor gravity. Mod Phys Lett A 2007; 22; 2647.

[67] Iorio L. The Measurement of Gravitomagnetism: A Challenging Enterprise, Hauppauge, New York: NOVA Science 2007.

[68] Ruffini R, Sigismondi C. Nonlinear Gravitodynamics: The LenseThirring Effect. Singapore: World Scientific 2003. 
[69] Mashhoon B, Hehl FW, Theiss DS. On the gravitational effects of rotating masses: The Thirring-Lense papers. Gen Rel Grav 1984; 16: 711 .

[70] Barker BM, O'Connell RF. Gravitational two-body problem with arbitrary masses, spins, and quadrupole moments. Phys Rev D 1974; 10: 1340.

[71] Ashby N, Allison T. Canonical planetary perturbation equations for velocity-dependent forces, and the Lense-Thirring precession. Celest Mech Dyn Astron 1993; 57: 537.

[72] Ruggiero ML, Tartaglia A. Gravitomagnetic effect. Nuovo Cim. 2002;B 117: 743. Tartaglia A. Gravitomagnetism, clocks and geometry. Eur J Phys 2001; 22:105.

[73] Kopeikin SM. Gravitomagnetism and the speed of gravity . Int Jou Mod Phys D 2006;15: 305.

[74] Barack L, Cutler C. LISA capture sources: Approximate waveforms, signal-to-noise ratios, and parameter estimation accuracy. Phys Rev D 2004; 69: 082005.

[75] Barack L, Cutler C. Confusion noise from LISA capture sources. Phys Rev D 2004; D 70: 122002.

[76] Einstein A. Approximative integration of the field equations of gravitation, Sitzungsberichte Preußische Akademie der Wissenschaften Berlin Math Phys 1916; 688

[77] Einstein A. On gravitational waves. Sitzungsberichte Preußische Akademie der Wissen-schaften Berlin Math Phys 1918; 154

[78] Kennefick D. Controversies in the history of the radiation reaction problem in general relativity. Unpublished Ph. D. thesis (Part II), California Institute of Technology 1997; short version available as gr-qc/9704002.

[79] Abbott B, Abbott R, Adhikari R, et al. Setting upper limits on the strength of periodic gravitational waves from PSR J1939+2134 using the first science data from the GEO 600 and LIGO detectors. Phys Rev D 2004;69: 082004.

[80] Abbott B, Abbott R, Adhikari R, et al. First upper limits from LIGO on gravitational wave bursts. Phys Rev D 2004; 69: 102001.

[81] Abbott B, Abbott R, Adhikari R, et al. Analysis of LIGO data for gravitational waves from binary neutron stars. Phys Rev D 2004; 69: 122001.

[82] Abbott B, Abbott R, Adhikari R, et al. Analysis of first LIGO science data for stochastic gravitational waves. Phys Rev D 2004; 69: 122004.

[83] Shapiro SL, Teukolsy SA. Black Holes, White dwarfs and Neutron Stars. Chicago, USA: Chicago University Press 1983.

[84] Van Bladel J. Lorenz or Lorentz?. IEEE Antennas Prop Mag 1991; 33: 69.

[85] Griffiths DJ. Introduction to Electrodynamics, $3^{\text {rd }}$ Ed. New Jersey: Prentice-Hall, Inc. 1999. Note, the footnote on "Lorenz vs. Lorentz" does not appear in the 1 st printing, but does appear in the 4th printing.

[86] Rakhamanov M. Phys Rev D 2005; 71: 084003.

[87] Larson SL, Hiscock WA, Hellings RW. Sensitivity curves for space borne gravitational wave interferometers. Phys Rev D 2000; 62: 062001.

[88] Corda C. A longitudinal component in massive gravitational waves arising from a bimetric theory of gravity. Astrop Phys 2007; 27 : 539.

[89] Corda C. The importance of the "magnetic" components of gravitational waves in the response functions of interferometers. Journal of Mod Phys D 2007; 16:1497. (a) Iorio L, Corda C. Gravitomagnetic effect in gravitational waves. AIP Conf Proc 2009;1168:1072. (b) Iorio L, Corda C. Gravitomagnetism and gravitational waves. Available from arXiv:1001.3951v2 [gr-qc].

[90] Corda C. Interferometric detection gravitational waves:the definitive test for general relativity. Int J Mod Phys D 2009; 18 : 2275.

[91] Hartle JB. Gravity: An introduction to Einstein's general relativity. San Francisco: Addison-Wesley 2003.

[92] Hughes SA, Thorne KS. Seismic gravity-gradient noise in interferometric gravitational-wave detectors. Phys Rev D 1998; 58: 122002.

[93] Thorne KS, Winstein CJ. Human gravity-gradient noise in interferometric gravitational-wave detectors. Phys Rev D 1999; 60: 082001 .

[94] Creighton T. Tumbleweeds and airborne gravitational noise sources for LIGO. Class Quant Grav 2008; 25:125011.

[95] Jackson JD. Classical Electrodynamics. Second Edition" New York: John Wiley \& Sons 1975; Sec. 6.6; or, "Classical
Electrodynamics, $3^{\text {rd }}$ Ed.", New York: John Wiley \& Sons 1999, Sec. 6.4.

[96] Thorne KS, Price RH, MacDonald DA. Black Holes: The Membrane Paradigm. New haven: Yale University Press 1986.

[97] Blanchet L. Post-Newtonian theory and the two-body problem. Living Rev Rel 2006; 9: 4.

[98] Damour T. The problem of motion in Newtonian and Einsteinian gravity, in 300 Years of Gravitation ed. by S.W. Hawking W. Israel Cambridge: Cambridge University Press 1987.

[99] Blanchet L. Gravitational radiation from post-Newtonian sources and inspiralling compact binaries. Living Rev Rel 2002; 5: 3.

[100] Jaranowski P, Schäfer G. Third post-Newtonian higher order ADM Hamilton dynamics for two-body point-mass systems. Phys Rev D 1998; 57:7274. (a) Blanchet L, Faye G, Iyer BR, Joguet B. Gravitational-wave inspiral of compact binary systems to $7 / 2$ postNewtonian order. Phys Rev D 2002; 65: 061501.

[101] Kidder L, Will C, Wiseman A. Spin effects in the inspiral of coalescing compact binaries. Phys Rev D 1993; 47: 4183. (a) Kidder L. Coalescing binary systems of compact objects to (post)5/2-Newtonian order. V. Spin effects. Phys Rev D 1995;52: 821. (b) Owen B, Tagoshi H, Ohashi A. Non precessional spinorbit effects on gravitational waves from inspiraling compact binaries to second post-Newtonian order. Phys Rev D 1998;57: 6168. (c) Tagoshi H, Ohashi A, Owen B. Gravitational field and equations of motion of spinning compact binaries to 2.5 postNewtonian order. Phys Rev D 2001;63, 044006. (d) Faye G, Blanchet L, Buonanno A. Higher-order spin effects in the dynamics of compact binaries. I. Equations of motion. Phys Rev D 2006;74: 104033. (e) Blanchet L, Buonanno A, Faye G. Higher-order spin effects in the dynamics of compact binaries. II. Radiation field. Phys Rev D 2006; 74: 104034.

[102] Mashhoon B. Stability of charged rotating black holes in the eikonal approximation. Phys Rev D 1985; 31: 290.

[103] Press W. Long Wave Trains of Gravitational Waves from a Vibrating Black Hole. Astrophys J Letters 1971; 170: L105.

[104] Davis M, Ruffini R, Tiomno J. Vector and Tensor Radiation from Schwarzschild Relativistic Circular Geodesics. Phys Rev D 1972; 5: 2932.

[105] Price RH, Pullin J. Colliding black holes: The close limit. Phys Rev Lett 1994;72: 3297. (a) Anninos P, Hobill D, Seidel E, Smarr L, Suen WM. Head-on collision of two equal mass black holes. Phys Rev Lett 1993;71: 2851. (b) Baker J, Abrahams A, Anninos P, Brandt S, Price R, Pullin J, Seidel E. Collision of boosted black holes. Phys Rev D 1997; 55: 829.

[106] Baker J, Brügmann B, Campanelli M, Lousto CO, Takahashi R. Plunge Waveforms from Inspiralling Binary Black Holes. Phys Rev Lett 2001;87: 121103.

[107] Buonanno A, Cook G, Pretorius F. Inspiral, merger and ring-down of equal-mass black-hole binaries. Phys Rev D 2007; 75 :124018.

[108] Baker J, van Meter J, McWilliams S, Centrella J, Kelly B. Consistency of post-Newtonian waveforms with numerical relativity. Phys Rev Lett 2007; 99:181101.

[109] Sigurdsson S. Estimating the detectable rate of capture of stellar mass black holes by massive central black holes in normal galaxies. Class Quant Grav 1997; 14: 1425.

[110] Danzmann K, Heinzel G, Reiche J, et al. LISA- Laser Interferometer Space Antenna, Pre-Phase A Report, Max-PlanckInstitut fur Quantenoptic,Report MPQ 1998, 233.

[111] Amaro-Seoane P, Gair JR, Coleman-Milleret M, et al. Intermediate and extreme mass-ratio inspirals Nastrophysics, science applications and detection using LISA. Class Quant Grav 2007; 24: R113.

[112] Thirring $H$. Uber die Wirkung rotierender ferner Massen in der Einsteinschen Gravitationstheorie. Phys Z 1918; 19: 204.

[113] Iorio L, Lainey V. The Lense-Thirring effect in the jovian system of the galilean satellites and its measurability. Int Jou Mod Phys D 2005; 14: 2039.

[114] Ryan FD. Accuracy of estimating the multipole moments of a massive body from the gravitational waves of a binary inspiral. Phys Rev D 1997; 561845.

[115] Freitag M. Gravitational Waves from Stars Orbiting the Sagittarius A* Black Hole. Astro J 2003; 583: L21.

[116] Capozziello S, De Laurentis M, Francaviglia M. Higher-order gravity and the cosmological background of gravitational waves. Astrop Phys 2008; 29: 125. 
[117] Rubbo LJ, Holley - Bockelmann K, Finn LS. Event Rate for Extreme Mass Ratio Burst Signals in the Laser Interferometer Space Antenna Band. Ap J 2006; 649 L25.

[118] Lightman AP, Shapiro SL. The dynamical evolution of globular clusters. Rev Mod Phys 1978; 50: 437.

[119] Genzel R, Townes CH. Clustering of Astronomers. Ann Rev Astron Astroph 1987; 25: 1 .

[120] Capozziello S, Lambiase G, Papini G, Scarpetta G. Higher Order Corrections in Gravitational Microlensing. Phys Lett 1999; A 254: 11

[121] Capozziello S, Re V. Higher-order corrections to lensing parameters for extended gravitational lenses. Phys Lett A 2001; A 290: 115 .

[122] Capozziello S, Cardone VF, Re V, Sereno M. Gravitomagnetic corrections to the lensing deflection angle for spiral galaxy models. Mon Not R Astr Soc 2003; 343: 360 .

[123] Sereno M, Cardone VF. Gravitational lensing by spherically symmetric lenses with angular momentum. Astron Astroph 2002; 396: 393.
[124] Levin J. Gravity Waves, Chaos, and Spinning Compact Binaries. Phys Rev Lett 2000; 843515.

[125] Gair JR, Barack L, Creighton T, et al. Event rate estimates for LISA extreme mass ratio capture sources. Class Quant Grav 2004; 21: S1595.

[126] Blanchet L. Gravitational radiation from post-Newtonian sources and inspiralling compact binaries. Living Rev Relativity 2002; 5: 3 .

[127] Poincaré H. Science et Methode, Book 1, chapt. II, p.34, Flammarion: Paris (1934). (a) Poincaré H. Les methodes nouvelles de la mecanique celeste, Paris: Gauthier-Villaris, 1892 reprinted by Librairie Albert Blanchard, Paris 1987.

[128] Tisserand F. Traitéde Mechanique Celeste, vol.I, Gauthier-Villaris, Paris (1960), (reprint of the first edition of 1889).

[129] Arnold VI. Mathematical Methods of Classical Mechanics. New York: Springer 1978

[130] Gallavotti G. The Element of Mechanics. New York: Springer 1983.

Received: May 20, 2011

Revised: June 08, 2011

Accepted: June 10, 2011

(C) Mariafelicia De Laurentis; Licensee Bentham Open.

This is an open access article licensed under the terms of the Creative Commons Attribution Non-Commercial License (http://creativecommons.org/licenses/by-nc/3.0/) which permits unrestricted, non-commercial use, distribution and reproduction in any medium, provided the work is properly cited. 